\title{
Article
}

\section{Nearby Early-type Galactic Nuclei at High Resolution: Dynamical Black Hole and Nuclear Star Cluster Mass Measurements}

Nguyen, Dieu D., Seth, Anil C., Neumayer, Nadine, Kamann, Sebastian, Voggel, Karina T., Cappellari, Michele, Picotti, Arianna, Nguyen, Phoung M., Boker, Torsten and Debattista, Victor P

Available at https://clok.uclan.ac.uk/28152/

Nguyen, Dieu D., Seth, Anil C., Neumayer, Nadine, Kamann, Sebastian, Voggel, Karina T., Cappellari, Michele, Picotti, Arianna, Nguyen, Phoung M., Boker, Torsten et al (2018) Nearby Early-type Galactic Nuclei at High Resolution: Dynamical Black Hole and Nuclear Star Cluster Mass Measurements. The Astrophysical Journal, 858 (2). ISSN 0004-637X

It is advisable to refer to the publisher's version if you intend to cite from the work. http://dx.doi.org/10.3847/1538-4357/aabe28

For more information about UCLan's research in this area go to http://www.uclan.ac.uk/researchgroups/ and search for <name of research Group>.

For information about Research generally at UCLan please go to http://www.uclan.ac.uk/research/

All outputs in CLoK are protected by Intellectual Property Rights law, including Copyright law. Copyright, IPR and Moral Rights for the works on this site are retained by the individual authors and/or other copyright owners. Terms and conditions for use of this material are defined in the policies page. 


\title{
Nearby Early-type Galactic Nuclei at High Resolution: Dynamical Black Hole and Nuclear Star Cluster Mass Measurements
}

\author{
Dieu D. Nguyen ${ }^{1}$ (1), Anil C. Seth ${ }^{1}$, Nadine Neumayer $^{2}$ (D), Sebastian Kamann ${ }^{3}$, Karina T. $\operatorname{Voggel}^{1}$ (D), Michele Cappellari ${ }^{4}$ (D), \\ Arianna Picotti ${ }^{2}$, Phuong M. Nguyen ${ }^{5}$, Torsten Böker ${ }^{6}$, Victor Debattista ${ }^{7}$ (i), Nelson Caldwell ${ }^{8}$ (D), Richard McDermid ${ }^{9}$, \\ Nathan Bastian ${ }^{3}$, Christopher C. Ahn ${ }^{1}$ (D), and Renuka Pechetti ${ }^{1}$ \\ ${ }^{1}$ Department of Physics and Astronomy, University of Utah, 115 South 1400 East, Salt Lake City, UT 84112, USA; dieu.nguyen@utah.edu, aseth@astro.utah.edu, \\ kvoggel@astro.utah.edu, renuka.pechetti@utah.edu, chris.ahn43@gmail.com \\ ${ }^{2}$ Max Planck Institut für Astronomie (MPIA), Königstuhl 17, D-69121 Heidelberg, Germany; neumayer@mpia.de, picotti@mpia.de \\ ${ }^{3}$ Astrophysics Research Institute, Liverpool John Moores University, 146 Brownlow Hill, Liverpool L3 5RF, UK; s.kamann@ljmu.ac.uk, N.J.Bastian@ljmu.ac.uk \\ ${ }^{4}$ Sub-department of Astrophysics, Department of Physics, University of Oxford, Denys Wilkinson Building, \\ Keble Road, Oxford OX1 3RH, UK; michele.cappellari@physics.ox.ac.uk
${ }^{5}$ Department of Physics, Quy Nhon University, 170 An Duong Vuong, Quy Nhon, Vietnam; nguyenthiminhphuong@qnu.edu.vn \\ ${ }^{6}$ European Space Agency, c/o STScI, 3700 San Martin Drive, Baltimore, MD 21218, USA; tboeker@ cosmos.esa.int \\ ${ }^{7}$ School of Physical Sciences and Computing, University of Central Lancashire, Preston, Lancashire, PR1 2HE, UK; vpdebattista@uclan.ac.uk \\ 8 Harvard-Smithsonian Center for Astrophysics, Harvard University, 60 Garden Street, Cambridge, MA 02138, USA; caldwell@cfa.harvard.edu \\ ${ }^{9}$ Department of Physics and Astronomy, Macquarie University, NSW 2109, Australia; richard.mcdermid@mq.edu.au \\ Received 2017 October 27; revised 2018 March 23; accepted 2018 April 11; published 2018 May 15
}

\begin{abstract}
We present a detailed study of the nuclear star clusters (NSCs) and massive black holes (BHs) of four of the nearest low-mass early-type galaxies: M32, NGC 205, NGC 5102, and NGC 5206. We measure the dynamical masses of both the BHs and NSCs in these galaxies using Gemini/NIFS or VLT/SINFONI stellar kinematics, Hubble Space Telescope (HST) imaging, and Jeans anisotropic models. We detect massive BHs in M32, NGC 5102, and NGC 5206, while in NGC 205, we find only an upper limit. These BH mass estimates are consistent with previous measurements in M32 and NGC 205, while those in NGC 5102 and NGC 5206 are estimated for the first time and both found to be $<10^{6} M_{\odot}$. This adds to just a handful of galaxies with dynamically measured sub-million $M_{\odot}$ central BHs. Combining these BH detections with our recent work on NGC 404's BH, we find that 80\% (4/5) of nearby, low-mass $\left(10^{9}-10^{10} M_{\odot} ; \sigma_{\star} \sim 20-70 \mathrm{~km} \mathrm{~s}^{-1}\right)$ early-type galaxies host BHs. Such a high occupation fraction suggests that the BH seeds formed in the early epoch of cosmic assembly likely resulted in abundant seeds, favoring a low-mass seed mechanism of the remnants, most likely from the first generation of massive stars. We find dynamical masses of the NSCs ranging from 2 to $73 \times 10^{6} M_{\odot}$ and compare these masses to scaling relations for NSCs based primarily on photometric mass estimates. Color gradients suggest that younger stellar populations lie at the centers of the NSCs in three of the four galaxies (NGC 205, NGC 5102, and NGC 5206), while the morphology of two are complex and best fit with multiple morphological components (NGC 5102 and NGC 5206). The NSC kinematics show they are rotating, especially in M32 and NGC $5102\left(V / \sigma_{\star} \sim 0.7\right)$.
\end{abstract}

Key words: galaxies: individual (NGC 221 (M32), NGC 205, NGC 5102, and NGC 5206) - galaxies: kinematics and dynamics - galaxies: nuclei

Supporting material: machine-readable tables

\section{Introduction}

Supermassive black holes (SMBHs) appear to be ubiquitous features of the centers of massive galaxies. This is inferred in nearby galaxies, both from their dynamical detection (see review by Kormendy \& Ho 2013) and from the presence of accretion signatures (e.g., Ho et al. 2009). Furthermore, the mass density of these BHs in nearby massive galaxies is compatible with the inferred mass accretion of active galactic nuclei (AGNs) in the distant universe (e.g., Marconi et al. 2004). However, the presence of central BHs is not well constrained in lower-mass galaxies, particularly below stellar masses of $\sim 10^{10} M_{\odot}$ (e.g., Greene 2012; Miller et al. 2015). These lower-mass galaxy nuclei are almost universally populated by bright, compact nuclear star clusters (NSCs; e.g., den Brok et al. 2014a; Georgiev \& Böker 2014) with a half-light/effective radius of $r_{\text {eff }} \sim 3 \mathrm{pc}$. These NSCs are known to coexist in some cases with massive BHs, including in the Milky Way (MW; Seth et al. 2008; Graham \& Spitler 2009; Neumayer \& Walcher 2012).
Finding and weighing central $\mathrm{BHs}$ in lower-mass galaxies is challenging due to the difficulty of dynamically detecting the low-mass $\left(\lesssim 10^{6} M_{\odot}\right)$ BHs they host. However, it is a key measurement for addressing several related science topics. First, low-mass galaxies are abundant (e.g., Blanton et al. 2005), and thus if they commonly host BHs, these will dominate the number density (but not mass density) of BHs in the local universe. This has consequences for a wide range of studies, from the expected rate of tidal disruptions (Kochanek 2016) to the number of BHs we expect to find in stripped galaxy nuclei (e.g., Pfeffer et al. 2014; Ahn et al. 2017). Second, the number of low-mass galaxies with central $\mathrm{BHs}$ is currently the most feasible way to probe the unknown formation mechanism of massive BHs. More specifically, if massive BHs form from the direct collapse of the relatively massive $\sim 10^{5} M_{\odot}$ seed $\mathrm{BH}$ scenario (e.g., Lodato \& Natarajan 2006; Bonoli et al. 2014), few would be expected to inhabit low-mass galaxies; if, on the other hand, they form from the remnants of Population III stars, a much higher "occupation fraction" is expected in low-mass galaxies (Volonteri et al. 2008; van Wassenhove et al. 2010; Volonteri 2010). This work is 
complementary to work that is starting to be undertaken at higher redshifts to probe accreting $\mathrm{BHs}$ at the earliest epochs (e.g., Weigel et al. 2015; Natarajan et al. 2017; Volonteri et al. 2017). Tidal disruptions are also starting to probe the $\mathrm{BH}$ population of low-mass galaxies (e.g., Law-Smith et al. 2017; Wevers et al. 2017) and may eventually provide constraints on occupation fraction (e.g., Stone \& Metzger 2016). Third, dynamical mass measurements of SMBHs $\left(M_{\mathrm{SMBH}} \sim 10^{6}-10^{10} M_{\odot}\right)$ have shown that their masses scale with the properties of their host galaxies, such as their bulge luminosity, bulge mass, and velocity dispersion (Kormendy \& Ho 2013; McConnell \& Ma 2013; Graham \& Scott 2015; Saglia et al. 2016). These scaling relations have been used to suggest that galaxies and their central SMBHs coevolve, likely due to feedback from the AGN radiation onto the surrounding gas (see review by Fabian 2012).

The BH-galaxy relations are especially tight for massive early-type galaxies (ETGs), while later-type, lower-dispersion galaxies show a much larger scatter (Greene et al. 2016; Läsker et al. 2016b). However, the lack of measurements at the lowmass, low-dispersion end, especially in ETGs, means that our knowledge of how BHs populate host galaxies is very incomplete.

The population of known BHs in low-mass galaxies was recently compiled by Reines \& Volonteri (2015). Of the BHs known in galaxies with stellar masses $<10^{10} M_{\odot}$, most have been found by the detection of optical broad-line emission, with their masses inferred from the velocity widths of their broad lines (e.g., Greene \& Ho 2007; Dong et al. 2012; Reines et al. 2013). Many of these galaxies host BHs with inferred masses below $10^{6} M_{\odot}$, especially those with stellar mass $\lesssim 3 \times 10^{9} M_{\odot}$. Broad-line emission is, however, found in only a tiny fraction $(<1 \%)$ of low-mass galaxies; other accretion signatures that are also useful for identifying BHs in low-mass galaxies include narrow-line emission (e.g., Moran et al. 2014), coronal emission in the mid-infrared (e.g., Satyapal et al. 2009), tidal-disruption events (e.g., Maksym et al. 2013), and hard X-ray emission (e.g., Miller et al. 2015; She et al. 2017).

The current record holder for the lowest-mass central $\mathrm{BH}$ in a late-type galaxy (LTG) is the broad-line AGN in RGG 118, with $M_{\star} \sim 2 \times 10^{9} M_{\odot}\left(M_{\mathrm{BH}}=5 \times 10^{4} M_{\odot}\right.$; Baldassare et al. 2015). The lowest-mass systems known to host central massive BHs are ultracompact dwarfs (UCDs), which are likely stripped galaxy nuclei (Seth et al. 2014; Ahn et al. 2017). Apart from these systems, the lowest-mass $\mathrm{BH}$-hosting galaxies are the $M_{\star} \sim 3 \times 10^{8} M_{\odot}$ early-type dwarf in Abell 1795, where a central $\mathrm{BH}$ is suggested by the detection of a tidal-disruption event (Maksym et al. 2013), and the similar-mass dwarf elliptical galaxy Pox 52, which hosts a broad-line AGN (Barth et al. 2004; Thornton et al. 2008). Currently, there are only a few galaxies with sub-million $M_{\odot}$ dynamical $\mathrm{BH}$ mass estimates, including NGC $4395\left(M_{\mathrm{BH}}=4_{-3}^{+8} \times 10^{5} M_{\odot}\right.$; den Brok et al. 2015), NGC $404\left(M_{\mathrm{BH}} \lesssim 1.5 \times 10^{5} M_{\odot}\right.$; Nguyen et al. 2017), and NGC $4414\left(M_{\mathrm{BH}} \lesssim 1.6 \times 10^{6} M_{\odot}\right.$; Thater et al. 2017).

Unlike BHs, the morphologies, stellar populations, and kinematics of NSCs provide an observable record for understanding mass accretion into the central parsecs of galaxies. The NSCs' stellar-mass accretion can be due to (1) the migration of massive star clusters formed at larger radii that then fall into the galactic center via dynamical friction (e.g., Lotz et al. 2001; Antonini 2013; Guillard et al. 2016) or (2) the in situ formation of stars from gas that fall into the nucleus (e.g., Seth et al. 2006; Antonini et al. 2015b). Observations suggest that the formation of NSCs is ongoing, as they typically have multiple populations (e.g., Rossa et al. 2006; Nguyen et al. 2017). Most studies on NSCs in ETGs have focused on galaxies in nearby galaxy clusters (Walcher et al. 2006); spectroscopic and photometric studies suggest that, typically, the NSCs in these ETGs are younger than their surrounding galaxy, especially in galaxies $\lesssim 2 \times 10^{9} M_{\odot}$ (e.g., Côté et al. 2006; Paudel et al. 2011; Krajnović et al. 2018; Spengler et al. 2017). There is also a clear change in the scaling relations between NSCs and galaxy luminosities and masses at about this mass, with the scaling being shallower at lower masses and steeper at higher masses (Scott et al. 2013; den Brok et al. 2014a; Spengler et al. 2017). This change, in addition to the flattening of more-luminous NSCs (Spengler et al. 2017), suggests a possible change in the formation mechanism of NSCs from migration to in situ formation. However, the NSC scaling relations for ETGs are based almost entirely on photometric estimates, with a significant sample of dynamical measurements available only for nearby late-type spirals (Walcher et al. 2005) and only two in ETG FCC 277 (Lyubenova et al. 2013) and NGC 404 (Nguyen et al. 2017).

The relationship between NSCs and BHs is not well understood. The sample of objects with both a detected NSC and a BH is quite limited (Seth et al. 2008; Graham \& Spitler 2009; Neumayer \& Walcher 2012; Georgiev et al. 2016), but even from this data it is clear that low-mass galaxy nuclei are typically dominated by NSCs, while high-mass galaxy nuclei are dominated by SMBHs. This transition could be due to a number of mechanisms, including the tidal disruption of infalling clusters (Ferrarese et al. 2006; Antonini et al. 2015a), the growth of $\mathrm{BH}$ seeds in more massive galaxies by tidal disruption of stars (Stone et al. 2017), or differences in feedback from star formation versus AGN accretion (Nayakshin et al. 2009). In addition, the formation of NSCs and BHs could be explicitly linked with NSCs creating the needed initial seed BHs during formation (e.g., Portegies Zwart et al. 2004) or strong gas inflow creating the NSC and BH simultaneously (Hopkins \& Quataert 2010). However, the existence of a $\mathrm{BH}$ around which an NSC appears to be currently forming in the nearby galaxy Henize 2-10 suggests that BHs can form independently of NSCs (Nguyen et al. 2014).

This work presents a dynamical study of the BHs and NSCs of four nearby low-mass ETGs, including the two brightest companions of Andromeda, M32 and NGC 205, and two companions of Cen A, NGC 5102 and NGC 5206. Of these four galaxies, previous $\mathrm{BH}$ mass estimates exist for two; the detection of $(2.5 \pm 0.5) \times 10^{6} M_{\odot} \mathrm{BH}$ in M32 (Verolme et al. 2002; van den Bosch \& de Zeeuw 2010) and the upper limit of $3.8 \times$ $10^{4} M_{\odot}($ at $3 \sigma)$ in NGC 205 (Valluri et al. 2005). In this paper, we use adaptive optics integral field spectroscopic measurements combined with dynamical modeling using carefully constructed mass models to constrain the BH and NSC masses in all four galaxies, including the first published constraints on the BHs of NGC 5102 and NGC 5206.

This paper is organized into nine sections. In Section 2, we describe the observations and data reduction. The four galaxies' properties are presented in Section 3, and we construct luminosity and mass models in Section 4. We present kinematics measurements of their nuclei in Section 5. We model these kinematics using Jeans models and present the $\mathrm{BH}$ constraints and uncertainties in Section 6. In Section 7, we analyze the properties of the NSCs and present our 
measurements of their masses. We discuss our results in Section 8 and conclude in Section 9.

\section{Data and Data Reduction}

\subsection{HST Imaging}

The Hubble Space Telescope (HST)/wide field and planetary camera 2 (WFPC2) planetary camera (PC) and the advanced camera for surveys/high-resolution camera (ACS/HRC) imaging we use in this work is summarized in Table 1. The nuclei of M32 and NGC 5206 are observed in the WFPC2 PC chip in the F555W and F814W filters. For NGC 205, we use the central ACS/HRC F555W and F814W images. For NGC 5102, we use more imaging of WFPC2, including two central saturated F450W and F560W, unsaturated F547M, and $\mathrm{H} \alpha$ emission F656N.

For the ACS/HRC data, we downloaded reduced, drizzled images from the $H S T /$ Hubble Legacy Archive (HLA). However, because the HLA images of the WFPC2 PC chip have a pixel scale that is downgraded by $10 \%$, we rereduce the images, which are downloaded from the Mikulski Archive for Space Telescope (MAST), using Astrodrizzle (Avila et al. 2012) to a final pixel scale of $0 . \prime 0445$. For all images, we constrain the sky background by comparing them to ground-based data (see Section 4.1).

We use the centroid positions of the nuclei to align images from all HST filters to the F814W (in M32, NGC 205, and NGC 5206) or F547M (in NGC 5102) data. The astrometric alignment of the Gemini near-infrared integral-field spectrograph (Gemini/NIFS) or Very Large Telescope/Spectrograph for Integral Field Observations in the Near Infrared (VLT/ SINFONI) spectroscopic data (Section 2.2) was then also tied to the same images, providing a common reference frame for all images used in this study.

\subsection{Integral Field Spectroscopic Data}

\subsubsection{Gemini/NIFS Spectroscopy}

M32 and NGC 205 were observed with Gemini/NIFS using the Altair tip-tilt laser guide star system. The M32 data were previously presented in Seth (2010). The observational information can be found in Table 2. We use these data to derive stellar kinematics from the $\mathrm{CO}$ band-head absorption in Section 5.

The data were reduced using the IRAF pipeline modified to propagate the error spectrum; for details, see Seth et al. (2010). The telluric calibration was done with A0V stars; for M32, HIP 116449 was used (Seth 2010), while for NGC 205, we use HIP 52877. The final cubes were constructed via a combination of six (M32) and eight (NGC 205) dithered on-source cubes with good image quality after subtracting offset sky exposures. The wavelength calibration used both an arc-lamp image and the sky lines in the science exposures with an absolute error of $\sim 2 \mathrm{~km} \mathrm{~s}^{-1}$.

\subsubsection{VLT/SINFONI Spectroscopy}

NGC 5102 and NGC 5206 were observed with SINFONI (Eisenhauer et al. 2003; Bonnet et al. 2004) on the UT4 (Yepun) of the European Southern Observatory's (ESO) VLT at Cerro Paranal, Chile. NGC 5102 was observed on two consecutive nights in March 2007 as part of the SINFONI GTO program (PI: Bender). A total of 12 on-source exposures of
$600 \mathrm{~s}$ integration time were observed in the two nights using the 100 mas pixel scale. NGC 5206 was observed in service mode in three different years (2011, 2013, and 2014; PI: Neumayer) with three on-source exposures of $600 \mathrm{~s}$ in each of the runs. Both of the targets were observed using the laser guide star for the adaptive optics correction and used the NSC itself for the tip-tilt correction. The spectra were taken in the $K$ band $(1.93-2.47 \mu \mathrm{m})$ at a spectral resolution of $R \sim 4000$, covering a field of view (FOV) of $3^{\prime \prime} \times 3^{\prime \prime}$. The data were reduced using the ESO SINFONI data reduction pipeline following the steps (1) sky subtraction, (2) flat-fielding, (3) bad-pixel correction, (4) distortion correction, (5) wavelength calibration, (6) cube reconstruction, and, finally, (8) telluric correction. Details of these steps are given in Neumayer et al. (2007). The final data cubes were constructed via a combination of the individual dithered cubes. This leads to a total exposure time of $7200 \mathrm{~s}$ for NGC 5102 and $5400 \mathrm{~s}$ for NGC 5206.

\subsection{Point-spread Function Determinations}

Our analysis requires careful characterization of the pointspread functions (PSFs) in both our kinematic and imaging data. The HST PSFs are used in fitting the two-dimensional (2D) surface brightness (SB) profiles of the galaxies (GALFIT; Peng et al. 2010), while the Gemini/NIFS and VLT/SINFONI PSFs are used for the dynamical modeling.

For the HST PSFs, we create the model PSF for each $H S T$ exposure using the Tiny Tim routine for each involved individual filter for each object. The PSFs are created using the tiny 1 and tiny2 tasks (Krist 1995; Krist et al. 2011). We next insert these into corresponding c0m (M32, NGC 5102, and NGC 5206) or flt (NGC 205) images at the positions of the nucleus in each individual exposure to simulate our observations and use Astrodrizzle to create our final PSF as described in den Brok et al. (2015) and Nguyen et al. (2017).

For the PSFs of our integral field spectra, we convolve the $H S T$ images with an additional broadening to determine the two-component PSF expected from adaptive optics observations. The Gemini/NIFS PSFs for M32 and NGC 205 are estimated as described in Seth et al. (2010). First, the outer shape of the PSF was constrained using images of the telluric calibrator Moffat profile $\left(\Sigma(r)=\Sigma_{0} /\left[\left(1+\left(r / r_{d}\right)^{2}\right)\right]^{4.765}\right)$. We then convolved the HST images with an inner Gaussian + outer Moffat function (with fixed shape but free amplitude) to best match the shape of the NIFS continuum image; we refer to these as $\mathrm{G}+\mathrm{M}$ PSFs. The full widths at half maximum (FWHMs) and light fractions of the components are presented in Table 3.

The VLT/SINFONI data cubes of NGC 5102 and NGC 5206 contain an apparent scattered-light component that affects our data in two ways: (1) it creates a structured nonstellar background in the spectra, which we subtract before measuring kinematics, and (2) it is uniform across the field and therefore creates a flat outer profile that cannot be explained with a reasonable PSF. Because this scattered light does not contribute to our kinematic measurements, we do our best to remove this before fitting the PSF. Unfortunately, this component is degenerate with the true PSF. To measure the level of this scattered light, we compare the SB of the SINFONI data to a scaled version of the HST. Because the level of the background in the SINFONI continuum image is much larger than the expected background, we simply subtract the difference from the HST background before fitting our PSFs. 
Table 1

$H S T /$ WFPC2 PC/WF3 and ACS/HRC Imaging

\begin{tabular}{|c|c|c|c|c|c|c|c|c|c|c|c|}
\hline $\begin{array}{l}\text { Object } \\
\text { (1) }\end{array}$ & $\begin{array}{c}(\mathrm{J} 2000) \\
(\mathrm{h} \mathrm{m} \mathrm{s}) \\
(2)\end{array}$ & $\begin{array}{c}(\mathrm{J} 2000) \\
\left(\begin{array}{c}\circ \prime \prime \prime \\
(3)\end{array}\right.\end{array}$ & $\begin{array}{l}\text { Camera } \\
\text { (4) }\end{array}$ & $\begin{array}{l}\text { Aperture } \\
(5)\end{array}$ & $\begin{array}{c}\text { UT Date } \\
\text { (6) }\end{array}$ & $\begin{array}{l}\text { PID } \\
\text { (7) }\end{array}$ & $\begin{array}{l}\text { Filter } \\
\text { (8) }\end{array}$ & $\begin{array}{c}\text { Exptime } \\
\text { (s) } \\
(9)\end{array}$ & $\begin{array}{c}\text { Pixel Scale } \\
\left(\operatorname{arcsec} \text { pixel }^{-1}\right) \\
(10)\end{array}$ & $\begin{array}{c}\text { Zero Point }^{\mathrm{a}} \\
\text { (mag) } \\
(11)\end{array}$ & $\begin{array}{c}A_{\lambda}{ }^{\mathrm{b}} \\
(\mathrm{mag}) \\
(12)\end{array}$ \\
\hline \multirow[t]{2}{*}{ M32 } & $00: 42: 38.37$ & $40: 51: 49.3$ & WFPC2 & PC1-FIX & 1994 Dec 26 & 5236 & F555W & $4 \times 24$ & 0.0445 & 24.664 & 0.047 \\
\hline & & & WFPC2 & PC1-FIX & 1994 Dec 26 & 5236 & F814W & $4 \times 24$ & 0.0445 & 23.758 & 0.026 \\
\hline \multirow{2}{*}{ NGC 205} & 00:40:22.0 & 41:41:07.1 & ACS/HRC & HRC & 2002 Sep 08 & 9448 & F555W & $4 \times 640$ & 0.0300 & 25.262 & 0.047 \\
\hline & & & ACS/HRC & HRC & 2002 Sep 08 & 9448 & F814W & $8 \times 305$ & 0.0300 & 24.861 & 0.026 \\
\hline \multirow[t]{4}{*}{ NGC 5102} & $13: 21: 55.96$ & $-36: 38: 13.0$ & WFPC2 & PC1-FIX & 1994 Sep 02 & 5400 & F547M & $8 \times 70$ & 0.0445 & 23.781 & 0.050 \\
\hline & & & WFPC2 & PC1-FIX & 1994 Sep 02 & 5400 & F450W ${ }^{\star}$ & $11 \times 590$ & 0.0445 & 24.106 & 0.058 \\
\hline & & & WFPC2 & PC1-FIX & 1994 Sep 02 & 5400 & F569W ${ }^{\star}$ & $11 \times 590$ & 0.0445 & 24.460 & 0.043 \\
\hline & & & WFPC2 & PC1-FIX & 2001 May 27 & 8591 & F656N & $5 \times 340$ & 0.0445 & 19.683 & 0.035 \\
\hline \multirow[t]{2}{*}{ NGC 5206} & 13:33:43.92 & $-48: 09: 05.0$ & WFPC2 & PC1-FIX & 1996 May 11 & 6814 & F555W & $6 \times 350$ & 0.0445 & 24.664 & 0.047 \\
\hline & & & WFPC2 & PC1-FIX & 1996 May 11 & 6814 & F814W & $6 \times 295$ & 0.0445 & 23.758 & 0.026 \\
\hline
\end{tabular}

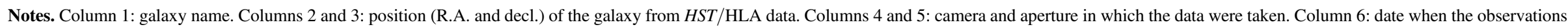

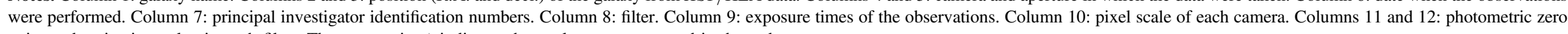
point and extinction value in each filter. The superscript ${ }^{\star}$ indicates the nucleus was saturated in these data.

${ }^{\mathrm{a}}$ The photometric zero points were based on the Vega system.

b The extinction values $A_{\lambda}$ were obtained from Schlafly \& Finkbeiner (2011) with interstellar extinction law from UV to near-infrared (Cardelli et al. 1989). 
Table 2

Gemini/NIFS and VLT/SINFONI Spectroscopic Data

\begin{tabular}{|c|c|c|c|c|c|c|c|c|c|}
\hline Object & $\begin{array}{l}\text { Instrument } \\
\text { (2) }\end{array}$ & $\begin{array}{c}\text { UT Date } \\
\text { (3) }\end{array}$ & $\begin{array}{c}\text { Mode/ } \\
\text { Telescope } \\
\text { (4) }\end{array}$ & $\begin{array}{l}\text { Exp. Time } \\
\text { (s) } \\
\text { (5) }\end{array}$ & $\begin{array}{l}\text { Sky } \\
\text { (6) }\end{array}$ & $\begin{array}{c}\text { Pixel Scale } \\
\left(\operatorname{arcsec} \text { pixel }^{-1}\right) \\
(7)\end{array}$ & $\begin{array}{l}\overline{\text { FWHM }} \\
(\AA) \\
(8)\end{array}$ & $\begin{array}{c}\overline{\text { FWHM }} \\
\left(\mathrm{km} \mathrm{s}^{-1}\right) \\
(9)\end{array}$ & $\begin{array}{l}\text { PID } \\
\text { (10) }\end{array}$ \\
\hline M32 & NIFS & 2005 Oct 23 & $\mathrm{AO}+\mathrm{NGS}$ & $6 \times 600$ & $5 \times 600$ & 0.05 & 4.2 & 57.0 & $\overline{\text { GN-2005B-SV-121 }}$ \\
\hline NGC 5102 & SINFONI & 2007 Mar 21 & UT4-Yepun & $12 \times 600$ & $12 \times 600$ & 0.05 & 6.2 & 82.2 & 078.B-0103(A) \\
\hline NGC 5206 & SINFONI & 2011 Apr 28 & UT4-Yepun & $3 \times 600$ & $3 \times 600$ & 0.05 & 6.2 & 82.2 & 086.B-0651(B) \\
\hline NGC 5206 & SINFONI & 2013 Jun 18 & UT4-Yepun & $3 \times 600$ & $3 \times 600$ & 0.05 & 6.2 & 82.2 & 091.B-0685(A) \\
\hline
\end{tabular}

Note. Column 1: galaxy name. Column 2: instrument used. Column 3: universal time date when the observations were processing. Column 4: additional information on $\mathrm{AO} /$ telescope used. Column 5: exposure times of the observations. Column 6: offset sky exposures used. Column 7: pixel scale of each camera. Columns 8 and 9: median FWHM (in wavelength and velocity) of the LSF. Column 10: program identification numbers.

Table 3

Gemini/NIFS and VLT/SINFONI PSF Models

\begin{tabular}{|c|c|c|c|c|}
\hline Object & $\begin{array}{l}\text { Observation } \\
\text { (2) }\end{array}$ & $\begin{array}{c}\text { Gaussian } 1 \\
(\operatorname{arcsec} / \text { frac.) } \\
\text { (3) }\end{array}$ & $\begin{array}{c}\text { Gaussian } 2 \\
\text { (arcsec/frac.) } \\
(4)\end{array}$ & $\begin{array}{c}\text { Moffat } \\
\text { (arcsec/frac.) } \\
\text { (5) }\end{array}$ \\
\hline $\mathrm{M} 32^{\mathrm{a}}$ & NIFS & $0.250 / 45 \%$ & $\ldots$ & $0.85 / 55 \%$ \\
\hline NGC 205 & NIFS & $0.093 / 68 \%$ & $\ldots$ & $0.92 / 32 \%$ \\
\hline $\begin{array}{l}\text { NGC } 5102 \\
\text { NGC } 5206\end{array}$ & $\begin{array}{l}\text { SINFONI } \\
\text { SINFONI }\end{array}$ & $\begin{array}{l}0.079 / 35 \% \\
0.117 / 60 \% \\
0.110 / 53 \%\end{array}$ & $\begin{array}{l}0.824 / 65 \%^{\mathrm{b}} \\
0.422 / 40 \%^{\mathrm{b}} \\
0.487 / 47 \%^{\mathrm{c}}\end{array}$ & $\begin{array}{l}\cdots \\
\cdots \\
\ldots\end{array}$ \\
\hline
\end{tabular}

Notes. Column 1: galaxy name. Column 2: instrument. Columns 3 and 4: FWHMs/light fractions of components of the kinematic PSFs. Column 5: Moffat profile $r_{d}$ (half-light radius)/light fraction.

${ }^{\text {a }}$ Seth (2010).

${ }^{\mathrm{b}}$ Scattered-light subtraction (default).

${ }^{\mathrm{c}}$ Half scattered-light subtraction.

However, as this likely results in somewhat of an oversubtraction, we also create an additional PSF where just half the original value was subtracted from the SINFONI data. This had a negligible effect on the PSF of NGC 5102; however, it was more significant for NGC 5206 ( $\triangle$ FWHM $\sim 18 \%$ ). We will discuss the impact of this PSF uncertainty on our dynamical modeling in Section 6.2.2. For the functional form of these PSFs, we test several functions and find that the best-fit PSFs are double Gaussians (2G); their FWHMs and light fractions are presented in Table 3 . We note that this is different from the NIFS PSF, which we found was better characterized by a Gauss+Moffat function for M32 and NGC 205.

\section{Galaxy Sample Properties}

Our sample of galaxies was chosen from known nucleated galaxies within $3.5 \mathrm{Mpc}$. We look at the completeness of this sample by examining the number of ETGs (numerical Hubble $T \leqslant 0$ ) with total stellar masses in the range $5 \times 10^{8} M_{\odot} \leqslant M_{\star} \leqslant 1 \times 10^{10} M_{\odot}$ using the updated nearby galaxy catalog (Karachentsev et al. 2013). To calculate the total stellar masses of galaxies in the catalog, we use their $K_{s}$-band luminosities and assume a uniform $M / L_{K} \sim 1\left(M_{\odot} / L_{\odot}\right)$ for our sample. Only five ETGs are in this stellar-mass range and within 3.5 Mpc, including the four in this work (M32, NGC 205, NGC 5102, and NGC 5206); the one other galaxy in the sample is NGC 404, which was the subject of our previous investigation (Nguyen et al. 2017). We note that within the same luminosity/mass and distance range, there are 17 total galaxies; the additional galaxies are all LTGs (including the LMC, M33, NGC 0055, and NGC 2403). Based on this, the four galaxies in this sample plus NGC 404 form a complete, unbiased sample of ETGs within 3.5 Mpc. However, we note that these ETGs live in a limited range of environments from isolated (NGC 404) to the Local Group and the Cen A group; thus, our sample does not include the dense cluster environment in which many ETGs live.

Before continuing with our analysis, we discuss the properties of the four galaxies in our sample in this section, summarizing the result in Table 4 . The listed total stellar masses are based on our morphological fits to a combination of ground-based and HST data, presented in Sections 4.1 and 4.2. Specifically, we take the total stellar luminosities of each best-fit component in our GALFIT models and multiply by a best-fit $M / L$ based on the color using the relations of Roediger \& Courteau (2015; hearafter R15); we note that using color $-M / L$ relations based on a Salpeter IMF gives higher masses by a factor of $\sim 2$ (e.g., Bell \& de Jong 2001; Bell et al. 2003). We consider these total masses to be accurate estimates for the bulge/spheroidal masses as well, as no significant outer disk components are seen in our fits. We note how these compare to previous estimates for each galaxy below.

\subsection{M32}

M32 is a dense dwarf elliptical or compact elliptical with the highest central density $\left(\rho>10^{7} M_{\odot} \mathrm{pc}^{-3}\right.$ within $r \lesssim 0.1 \mathrm{pc}$; Lauer et al. 1998) in the Local Group. It has a prominent NSC component visible in the SB profile at radii $\lesssim 5^{\prime \prime}(20 \mathrm{pc})$ that accounts for $\sim 10 \%$ of the total mass of the stellar bulge (Graham \& Spitler 2009). This NSC light fraction is much larger than typical ETG NSCs (e.g., Côté et al. 2006). Despite this clear SB feature, there is no evidence of a break in stellar kinematics or populations (Seth 2010). Specifically, the rotational velocity decreases slowly with radius (Dressler \& Richstone 1988; Seth 2010), while the stellar population age increases gradually outward, with average ages from 3 (nucleus) to 8 (large radii) Gyr with metallicity values at the center of $[\mathrm{Fe} / \mathrm{H}]=0-0.16$ and declining outward (Worthey 2004; Rose et al. 2005; Coelho et al. 2009; Villaume et al. 2017).

The central BH has previously been measured using SAURON stellar kinematics combined with STIS kinematics by Verolme et al. (2002). Their axisymmetric Schwarzschild code gives a best-fit mass of $M_{\mathrm{BH}}=(2.5 \pm 0.5) \times 10^{6} M_{\odot}$. 
Table 4

Host Galaxy Properties

\begin{tabular}{|c|c|c|c|c|c|}
\hline & M32 & NGC 205 & NGC 5102 & NGC 5206 & Unit \\
\hline Distance & $0.79[1]$ & $0.82[2]$ & $3.2[3]$ & $3.5[4]$ & $(\mathrm{Mpc})$ \\
\hline$m-M$ & 24.49 & 24.75 & 27.52 & 27.72 & (mag) \\
\hline Physical scale & 4.0 & 4.3 & 16.0 & 17.0 & $\left(\mathrm{pc} \operatorname{arcsec}^{-1}\right)$ \\
\hline Photometric total stellar mass & $1.00 \times 10^{9}[\star]$ & $9.74 \times 10^{8}[\star]$ & $6.0 \times 10^{9}[\star]$ & $2.4 \times 10^{9}[\star]$ & $\left(M_{\odot}\right)$ \\
\hline Dynamical total stellar mass & $1.08 \times 10^{9}[\star]$ & $1.07 \times 10^{9}[\star]$ & $6.9 \times 10^{9}[\star]$ & $2.5 \times 10^{9}[\star]$ & $\left(M_{\odot}\right)$ \\
\hline Effective radius ( $\mathrm{r}_{\text {eff. }}^{\text {galaxy }}$ ) & $30^{\prime \prime} / 120[8, \star]$ & $121^{\prime \prime} / 520[9, \star]$ & $75^{\prime \prime} / 1200[10,11, \star]$ & $58^{\prime \prime} / 986[\star]$ & $(\operatorname{arcsec}$ or $\mathrm{pc})$ \\
\hline Inclination & $70.0[12]$ & $\ldots$ & $86.0[12]$ & $\ldots$ & (deg) \\
\hline
\end{tabular}

Note. The subscripts ${ }_{B, V, I}$ indicate the measurements in the $B, V$, and $I$ bands. NED: NASA/IPAC Extragalactic Database. [CGS]: indicates the Carnegie-Irvine Galaxy Survey color profiles for total stellar masses assuming R15 color- $M / L$ relation. References: [1] Welch et al. (1986); [2] McConnachie et al. (2005); [3] van den Bergh (1976); [4] Tully et al. (2015); [5] http://leda.univ-lyon1.fr/search.html; [6] Seth (2010); [7] Geha et al. (2006); [8] Graham (2002); [9] De Rijcke et al. (2006), [10] Jarrett et al. (2003, LGA); [11] Davidge (2008); [12] Verolme et al. (2002); [ $\star$ ] estimates from this work. To calculate properties from multiple references, we take their mean.

This value was accurately and independently confirmed by van den Bosch \& de Zeeuw (2010) using a triaxial Schwarzschild modeling code. Accretion onto this $\mathrm{BH}$ is indicated by a faint X-ray and radio source (Ho et al. 2003; Yang et al. 2015), which suggests the source is accreting at $\sim 10^{-9}$ of its Eddington limit. However, Seth (2010) found nuclear emission from hot dust in the $K$ band with a luminosity more than $100 \times$ the nuclear X-ray luminosity.

Our total stellar luminosity in the $I$ band is $5.5 \times 10^{8} L_{\odot}$, and we estimate an average photometric $M / L_{I}=1.7$ based on the color $-M / L$ relation $\mathrm{R} 15$ of the full galaxy. This gives a photometric stellar mass of the galaxy/bulge of $9.4 \times 10^{8} M_{\odot}$ (Section 4.2 and Table 5). This photometric estimate is in relatively good agreement with the previous dynamical estimates (Richstone \& Sargent 1972; Häring \& Rix 2004; Kormendy \& Ho 2013). In Graham \& Spitler (2009), the bulge luminosity is $\sim 38 \%$ lower due possibly to separation of a disk component dominating their fits at radii $>100^{\prime \prime}$; combined with the much lower $M / L\left(M / L_{I}=0.92\right)$ they assume based on the result from Coelho et al. (2009), their bulge mass is just $2.6 \times 10^{8} M_{\odot}$.

\subsection{NGC 205}

NGC 205 is a nucleated dwarf elliptical galaxy hosting stars with a range of ages (Davidge 2005; Sharina et al. 2006). The $H S T$ observations of the inner $29^{\prime \prime} \times 26^{\prime \prime}$ around the nucleus of NGC 205 show a population of bright blue stars with ages ranging from 60 to $400 \mathrm{Myr}$ and a metallicity $[M / H] \sim-0.5$ (Monaco et al. 2009). This confirms that multiple star formation episodes have occurred within the central $\sim 100 \mathrm{pc}$ of the galaxy (Cappellari et al. 1999; Davidge 2003).

Valluri et al. (2005) and Geha et al. (2006) used STIS spectra of the Ca II absorption triplet region to measure the NGC 205 nuclear kinematics. They found no rotation and a flat dispersion of $\sim 20 \mathrm{~km} \mathrm{~s}^{-1}$ within the central 0 "! $3(\sim 1 \mathrm{pc})$. The combination of HST images, STIS spectra of the NGC 205 nucleus, and three-integral axisymmetric dynamical models give an upperlimit mass of a putative $\mathrm{BH}$ at the center of the galaxy of $\sim 3.8 \times 10^{4} M_{\odot}$ (within $3 \sigma$; Valluri et al. 2005)

Our total stellar luminosity in the $I$ band is $5.6 \times 10^{8} L_{\odot}$, and we estimate an average photometric $M / L_{I}$ of the whole galactic body based on the color- $M / L$ relation $\mathrm{R} 15$ of $1.8\left(M_{\odot} / L_{\odot}\right)$ and photometric stellar mass of $9.8 \times 10^{8} M_{\odot}$ (Section 4.2 and Table 5). The total mass within $\sim 1 \mathrm{kpc}\left(2 R_{e}\right)$ was dynamically estimated to be $1.0 \times 10^{9} M_{\odot}$ by De Rijcke et al. (2006), with the stellar mass making up $\sim 60 \%$ of this mass and thus matching quite closely our stellar-mass estimate. De Rijcke et al. (2006) also tracked the kinematics of the galaxy out to $1.2 \mathrm{kpc}$ and found that the dispersion slowly rises from $\sim 30$ to $45 \mathrm{~km} \mathrm{~s}^{-1}$, with rotation in the outer regions up to $\sim 20 \mathrm{~km} \mathrm{~s}^{-1}$.

\subsection{NGC 5102}

NGC 5102 is an S0 post-starburst galaxy in the nearby Cen A group (Deharveng et al. 1997; Davidge 2008). It has an H I disk extending to $>5^{\prime}(\sim 4.8 \mathrm{kpc}$; van Woerden et al. 1996), as well as extended ionized gas and dust emission (McMillan et al. 1994; Xilouris et al. 2004). Resolved stars and spectral synthesis studies show the presence of young stars and a significant intermediate-age ( $\sim 100 \mathrm{Myr}-3 \mathrm{Gyr})$ population that appears to dominate the stellar mass near the center, with the mean age growing with radius (Kraft et al. 2005; Davidge 2008, 2015; Mitzkus et al. 2017). Mitzkus et al. (2017) found that the nucleus has a mass-weighted age of $0.8 \mathrm{Gyr}$, with the best fit suggesting a young, 300 Myr solarmetallicity population overlying an old, metal-poor population $(>10 \mathrm{Gyr},[\mathrm{Fe} / \mathrm{H}]<-1)$.

Mitzkus et al. (2017) also measured the kinematics of NGC $5102\left(r \lesssim 30^{\prime \prime}\right)$ with MUSE data and found a flat dispersion of $\sim 44 \mathrm{~km} \mathrm{~s}^{-1}$ at radii larger than 0.5 and a dispersion peak of $60 \mathrm{~km} \mathrm{~s}^{-1}$ at the center. They also found a maximum rotation amplitude of $20 \mathrm{~km} \mathrm{~s}^{-1}$ at a radius of $10^{\prime \prime}$. A key characteristic of this galaxy is that it has two clear counterrotating disks (Mitzkus et al. 2017); this results in two dispersion peaks, making it a " $2 \sigma$ " galaxy.

A nuclear X-ray point source was detected by Kraft et al. (2005); this point source has a luminosity of $\sim 10^{37} \mathrm{erg} \mathrm{s}^{-1}$ in the $0.5-2.0 \mathrm{keV}$ band. No previous estimates exist for its central BH mass.

Our total stellar luminosity in the $V$ band is $2.2 \times 10^{9} L_{\odot}$, and we estimate an average photometric $M / L_{V}$ of the whole galactic body based on the color $-M / L$ relation R15 of $2.7\left(M_{\odot} / L_{\odot}\right)$ and a photometric stellar mass of $6.0 \times 10^{9} M_{\odot}$ (Section 4.2 and Table 5); this mass is $14 \%$ lower than the previous estimates of the galaxy total stellar mass (Davidge 2008). 
Table 5

GALFIT Models, Stellar Population Masses, and Luminosities of Galaxies in the Sample

\begin{tabular}{|c|c|c|c|c|c|c|c|c|c|c|c|c|}
\hline Object & $\begin{array}{l}\text { Filter } \\
\text { (2) }\end{array}$ & $\begin{array}{l}\text { SB } \\
\text { (3) }\end{array}$ & $\begin{array}{l}n_{i} \\
\text { (4) }\end{array}$ & $\begin{array}{c}r_{\mathrm{eff}, . i} \\
(\operatorname{arcsec}) \\
(5)\end{array}$ & $\begin{array}{l}r_{\text {eff } ., i} \\
(\mathrm{pc}) \\
(6)\end{array}$ & $\begin{array}{c}m_{i} \\
(\mathrm{mag}) \\
(7)\end{array}$ & $\begin{array}{c}\mathrm{PA}_{i} \\
(\mathrm{deg}) \\
(8)\end{array}$ & $\begin{array}{l}b / a_{i} \\
(9)\end{array}$ & $\begin{array}{l}\chi_{\mathrm{r}}^{2} \\
(10)\end{array}$ & $\begin{array}{c}L_{\star, i} \\
\left(\times 10^{7} L_{\odot}\right) \\
(11)\end{array}$ & $\begin{array}{c}M_{\star, i} \text {,pop. } \\
\left(\times 10^{7} M_{\odot}\right) \\
(12)\end{array}$ & $\begin{array}{l}\text { Comp. } \\
\text { (13) }\end{array}$ \\
\hline (1) & & \multicolumn{11}{|c|}{ Free PA } \\
\hline \multirow[t]{4}{*}{ M32 } & F814W & $2 \mathrm{D}$ & $2.7 \pm 0.3$ & $1.1 \pm 0.1$ & $4.4 \pm 0.4$ & $11.0 \pm 0.1$ & $-23.4 \pm 0.5$ & $0.75 \pm 0.03$ & & $1.10 \pm 0.10$ & $1.45 \pm 0.24$ & NSC \\
\hline & & $2 \mathrm{D}$ & $1.6 \pm 0.1$ & $27.0 \pm 1.0$ & $108 \pm 4$ & $7.0 \pm 0.1$ & $-24.7 \pm 0.4$ & $0.79 \pm 0.07$ & 1.7 & $43.5 \pm 4.0$ & $79.4 \pm 10.3$ & Bulge \\
\hline & & $1 \mathrm{D}^{\star}$ & 1 & 129 & 516 & 8.6 & $-25.0 \pm 0.7$ & $0.79 \pm 0.05$ & & 9.96 & $19.3 \pm 2.5$ & Disk \\
\hline & & \multicolumn{11}{|c|}{ Fixed PA } \\
\hline \multirow[t]{4}{*}{ M32 } & F814W & $2 \mathrm{D}$ & $2.7 \pm 0.3$ & $1.1 \pm 0.1$ & $4.4 \pm 0.4$ & $11.1 \pm 0.1$ & -25.0 & $0.75 \pm 0.09$ & & $1.10 \pm 0.10$ & $1.45 \pm 0.25$ & NSC \\
\hline & & $2 \mathrm{D}$ & $1.6 \pm 0.1$ & $27.0 \pm 1.0$ & $108 \pm 4$ & $7.1 \pm 0.1$ & -25.0 & $0.79 \pm 0.11$ & 2.0 & $43.8 \pm 4.1$ & $78.0 \pm 10.4$ & Bulge \\
\hline & & $1 \mathrm{D}^{\star}$ & 1 & 129 & 516 & 8.6 & -25.0 & $0.79 \pm 0.08$ & & 10.00 & $19.3 \pm 2.5$ & Disk \\
\hline & & \multicolumn{11}{|c|}{ Free PA } \\
\hline \multirow[t]{3}{*}{ NGC 205} & F814W & $2 \mathrm{D}$ & $1.6 \pm 0.2$ & $0.3 \pm 0.1$ & $1.3 \pm 0.4$ & $13.6 \pm 0.4$ & $-37.1 \pm 1.4$ & $0.95 \pm 0.03$ & & $0.10 \pm 0.04$ & $0.18 \pm 0.08$ & NSC \\
\hline & & $1 \mathrm{D}^{\star}$ & 1.4 & 120 & 516 & 6.8 & $-40.4 \pm 1.0$ & $0.90 \pm 0.07$ & 5.5 & 55.7 & $97.2 \pm 15.2$ & Bulge \\
\hline & & \multicolumn{11}{|c|}{ Fixed PA } \\
\hline \multirow[t]{3}{*}{ NGC 205} & F814W & $2 \mathrm{D}$ & $1.6 \pm 0.2$ & $0.3 \pm 0.1$ & $1.3 \pm 0.4$ & $13.6 \pm 0.4$ & -40.4 & $0.95 \pm 0.06$ & & $0.10 \pm 0.04$ & $0.18 \pm 0.08$ & NSC \\
\hline & & $1 \mathrm{D}^{\star}$ & 1.4 & 120 & 516 & 6.8 & -40.4 & $0.91 \pm 0.05$ & 5.9 & 55.7 & $97.2 \pm 15.2$ & Bulge \\
\hline & & \multicolumn{11}{|c|}{ Free PA } \\
\hline \multirow[t]{4}{*}{ NGC 5102} & F547M & $2 \mathrm{D}$ & $0.8 \pm 0.2$ & $0.1 \pm 0.1$ & $1.6 \pm 1.6$ & $14.20 \pm 0.30$ & $55.1 \pm 1.5$ & $0.68 \pm 0.06$ & & $1.81 \pm 0.51$ & $0.71 \pm 0.22$ & $\mathrm{NSC}_{1}$ \\
\hline & & $2 \mathrm{D}$ & $3.1 \pm 0.1$ & $2.0 \pm 0.3$ & $32.0 \pm 4.8$ & $12.27 \pm 0.21$ & $51.1 \pm 1.7$ & $0.59 \pm 0.04$ & 3.3 & $10.7 \pm 1.98$ & $5.8 \pm 0.6$ & $\mathrm{NSC}_{2}$ \\
\hline & & $1 \mathrm{D}^{\star}$ & 3 & 75 & 1200 & 9.05 & 50.0 & $0.60 \pm 0.07$ & & 210 & $592 \pm 83$ & Bulge \\
\hline & & \multicolumn{11}{|c|}{ Fixed PA } \\
\hline \multirow[t]{4}{*}{ NGC 5102} & F547M & $2 \mathrm{D}$ & $0.8 \pm 0.2$ & $0.1 \pm 0.1$ & $1.6 \pm 1.6$ & $14.20 \pm 0.30$ & 50.5 & $0.68 \pm 0.05$ & & $1.81 \pm 0.51$ & $0.71 \pm 0.22$ & $\mathrm{NSC}_{1}$ \\
\hline & & $2 \mathrm{D}$ & $3.1 \pm 0.1$ & $2.0 \pm 0.3$ & $32.0 \pm 4.8$ & $12.27 \pm 0.22$ & 50.5 & $0.60 \pm 0.08$ & 3.6 & $10.7 \pm 1.98$ & $5.8 \pm 0.6$ & $\mathrm{NSC}_{2}$ \\
\hline & & $1 \mathrm{D}^{\star}$ & 3 & 75 & 1200 & 9.05 & 50.5 & $0.63 \pm 0.10$ & & 210 & $592 \pm 83$ & Bulge \\
\hline & & \multicolumn{11}{|c|}{ Free PA } \\
\hline \multirow[t]{4}{*}{ NGC 5206} & F814W & $2 \mathrm{D}$ & $0.8 \pm 0.1$ & $0.2 \pm 0.1$ & $3.4 \pm 1.7$ & $16.9 \pm 0.5$ & $36.0 \pm 0.1$ & $0.96 \pm 0.03$ & & $0.094 \pm 0.045$ & $0.17 \pm 0.10$ & $\mathrm{NSC}_{1}$ \\
\hline & & $2 \mathrm{D}$ & $2.3 \pm 0.3$ & $0.6 \pm 0.1$ & $10.5 \pm 1.7$ & $14.8 \pm 0.2$ & $38.5 \pm 1.4$ & $0.96 \pm 0.02$ & 2.4 & $0.65 \pm 0.12$ & $1.28 \pm 0.6$ & $\mathrm{NSC}_{2}$ \\
\hline & & $1 \mathrm{D}^{\star}$ & 2.57 & 58 & 986 & 10.1 & 38.6 & $0.98 \pm 0.01$ & & 123.3 & $241 \pm 47$ & Bulge \\
\hline & & \multicolumn{11}{|c|}{ Fixed PA } \\
\hline \multirow[t]{3}{*}{ NGC 5206} & F814W & $2 \mathrm{D}$ & $0.8 \pm 0.1$ & $0.2 \pm 0.1$ & $3.4 \pm 1.7$ & $16.9 \pm 0.5$ & 38.3 & $0.96 \pm 0.03$ & & $0.094 \pm 0.045$ & $0.17 \pm 0.10$ & $\mathrm{NSC}_{1}$ \\
\hline & & $2 \mathrm{D}$ & $2.3 \pm 0.3$ & $0.6 \pm 0.1$ & $10.2 \pm 1.7$ & $14.8 \pm 0.2$ & 38.3 & $0.97 \pm 0.03$ & 2.7 & $0.65 \pm 0.12$ & $1.28 \pm 0.27$ & $\mathrm{NSC}_{2}$ \\
\hline & & $1 \mathrm{D}^{\star}$ & 2.57 & 58 & 986 & 9.1 & 38.3 & $0.98 \pm 0.01$ & & 122.3 & $241 \pm 47$ & Bulge \\
\hline
\end{tabular}

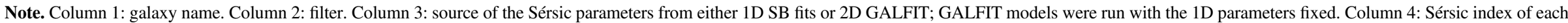

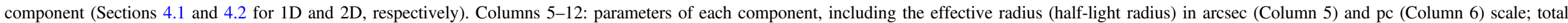

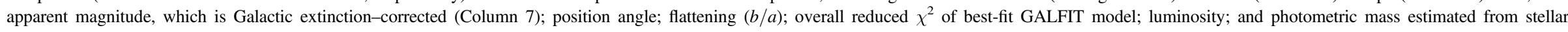

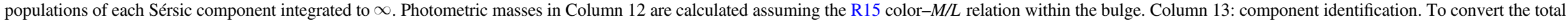

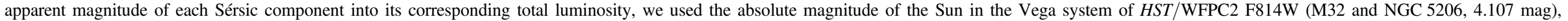
HST/WFPC2 F555W (approximate F547M for NGC 5102, 4.820 mag), and HST/HRC ACS F814W (NGC 205, 4.096 mag). (Available at http://www.baryons.org/ezgal/filters.php.) 


\subsection{NGC 5206}

NGC 5206 is a poorly studied dE/S0 galaxy in the Cen A group. It was previously found by Caldwell \& Bothun (1987) that the structure of the galaxy was not well fit just by a nucleus and a single component. The Carnegie-Irvine color profiles show that it has a bluer center, suggesting younger stellar populations (Li et al. 2011), while spectral synthesis fits to Xshooter data of its nucleus (N. Karcharov et al. 2018, in preparation) suggest a wide range of ages in the NSC, with the most recently formed stars being a population of solar-metallicity stars formed $\sim 1 \mathrm{Gyr}$ ago. A previous measurement has found a central velocity dispersion of $39 \pm 5 \mathrm{~km} \mathrm{~s}^{-1}$ (Peterson \& Caldwell 1993) and $41.5 \pm 6.5 \mathrm{~km} \mathrm{~s}^{-1}$ (Wegner et al. 2003).

Our total stellar luminosity in the $I$ band is $1.2 \times 10^{9} L_{\odot}$, and we estimate an average photometric dynamical $M / L_{I}$ of the whole galactic body based on the color $-M / L$ relation R15 of $1.98\left(M_{\odot} / L_{\odot}\right)$ and photometric stellar mass of $2.4 \times 10^{9} M_{\odot}($ Section 4.2 and Table 5$)$.

\section{SB Profiles}

\subsection{Large-scale Structure from the 1D SB Profiles}

To characterize the NSCs and create mass models for our targets, we first investigate the central SB profiles using $H S T$ imaging combined with larger-scale SB profiles from the ground-based data. After fitting for the large-scale properties of the galaxy, we fit the smaller-scale structure near the center in Section 4.2 using GALFIT.

For our 1D profiles, we use the IRAF ellipse (Jedrzejewski 1987) routine to extract fluxes in the annuli as a function of the major semiaxis. While extracting the fluxes, we allow the position angle (PA) and ellipticity $(\epsilon)$ to vary.

Due to the small FOV of the HST data, the sky background is not easy to estimate. To solve this problem, we use the existing ground-based data to estimate the sky backgrounds; the existing ground-based data include Kent (1987) and Lauer et al. (1998) for M32, Kent (1987) and Valluri et al. (2005) for NGC 205, and the Carnegie-Irvine Galaxy Survey (CGS; Ho et al. 2011; Li et al. 2011; Huang et al. 2013) for NGC 5102 and NGC 5206. We use data at intermediate radii to match the $H S T$ and ground-based SBs with shifts of $<0.15$ mag. Next, we determine the sky background in the HST images by matching them out to larger radii. In the end, our 1D SB profiles are calibrated in Vega magnitudes (Sirianni et al. 2005) and corrected for Galactic extinction (Table 1).

We fit the combined 1D SB profiles of each galaxy with multiple Sérsic profiles using the nonlinear least-squares IDL MPFIT function (Markwardt 2009). ${ }^{10}$ Because we do not do PSF convolution, we use these fits to constrain just the outer components of the fit; the best fits are shown in Figure 1 and Table 5. The NGC 205 SB profiles are well fit by a double Sérsic function (NSC + galaxy); however, the 1D SB of M32 requires two outer Sérsic profiles (the outermost Sérsic is an exponential) + NSC, while NGC 5102 and NGC 5206 require two NSC Sérsic components + a galaxy component based on their 2D fits. We note that our exponential disk component of M32 and the outer Sérsic component of NGC 205 are fully consistent with Graham (2002) and Graham \& Spitler (2009), and these components will be fixed in 2D GALFIT (Section 4.2). The robustness of our $1 \mathrm{D}$ fits is tested by

\footnotetext{
${ }^{10}$ Available from http://purl.com/net/mpfit.
}

changing the outer boundaries in the ranges of $70^{\prime \prime}-90^{\prime \prime}, 100^{\prime \prime}-$ $200^{\prime \prime}, 90^{\prime \prime}-110^{\prime \prime}$, and $90^{\prime \prime}-120^{\prime \prime}$ for M32, NGC 205, NGC 5102, and NGC 5206, respectively. The standard deviation of the Sérsic parameters from these fits was used to determine the errors; these errors are $<10 \%$ in all cases. The fits are performed in multiple bands, enabling us to model the color variation.

We show the 1D SB profile fits, residuals, and $(V-I)_{\mathrm{o}}$ color of each galaxy in the top, middle, and bottom panel of each plot in Figure 1. These models agree well within the data with MEAN (ABS ( (data-model) / data) $<5 \%$ for all four galaxies. M32 shows no radial color gradient, consistent with previous observations (Lauer et al. 1998). The other three galaxies show bluer colors toward their centers. We also note that we use these models only to constrain the outer Sérsic components of the galaxies; the best-fit inner components are derived from 2D modeling of the HST images.

\subsection{NSC Morphology from 2D GALFIT Models}

Our dynamical models rely on the accurate measurements of the 2D stellar-mass distribution near the centers of each galaxy. Moreover, the 2D SB profile is also important for quantifying the morphology of the NSCs. We model the HST images around the nucleus using GALFIT. GALFIT enables fitting with convolved models (using a PSF from Tiny Tim; Section 2.3) and excluding pixels using a bad-pixel mask. The bad-pixel mask is obtained using an initial GALFIT run without a mask; we mask all pixels with absolute pixel values $>3 \sigma$ than the median value in the residual images (Data-Model). Based on our 1D SB profile, we chose to fit NGC 205 with a double Sérsic function $(2 \mathrm{~S})$; M32 is fitted with a $2 \mathrm{~S}+$ exponential disk $(2 \mathrm{~S}+\mathrm{E})$. For NGC 5102 and NGC 5206, we find that the nuclear regions require two components (Figure 2), and thus these are fitted with triple Sérsic functions (3S). The initial guesses were input with the best-fit parameters from the 1D SB fits with fixed parameters for the outermost Sérsic or E component, except for the PA and axis ratio $(b / a)$. For the purpose of creating mass models for dynamical modeling of $\mathrm{BH}$ mass measurements, we repeat these 2D fits with a fixed PA in all Sérsic components because the Jeans anisotropic models (JAMs; Cappellari 2008) assume axisymmetry, which implies a constant PA.

The left column of Figure 2 shows the F814W images of M32, NGC 205, and NGC 5206 and F547M image of NGC 5102, while the middle column shows the relative errors between the HST images and their $2 \mathrm{~S}$ models, (DataModel) / Data. The right column also shows these relative errors between the HST images and their $2 \mathrm{~S}+\mathrm{E}$ (M32) or $3 \mathrm{~S}$ (NGC 5102 and NGC 5206) models. The contours in each panel show both data (black) and model (white) at the same radius and flux level to highlight the regions of agreement and disagreement between the data and models. Excluding masked regions (and point sources in NGC 205), the maximum errors on the individual fits are $<9 \%, 15 \%, 13 \%$, and $10 \%$ for M32, NGC 205, NGC 5102, and NGC 5206, respectively. The parameters of the best-fit GALFIT models are shown in Columns 4-9 of Table 5; errors are scaled based on the 1D errors in the components estimated in Section 4.1 due to their dominance over the GALFIT errors. We note that the 2D GALFIT models give Sérsic parameters consistent with those of the 1D SB profile fit, especially for the middle Sérsic components where the PSF effects are minimal. We note that our NSC component in M32 is significantly less luminous than the 1D SB profile given in Graham \& Spitler (2009); we 

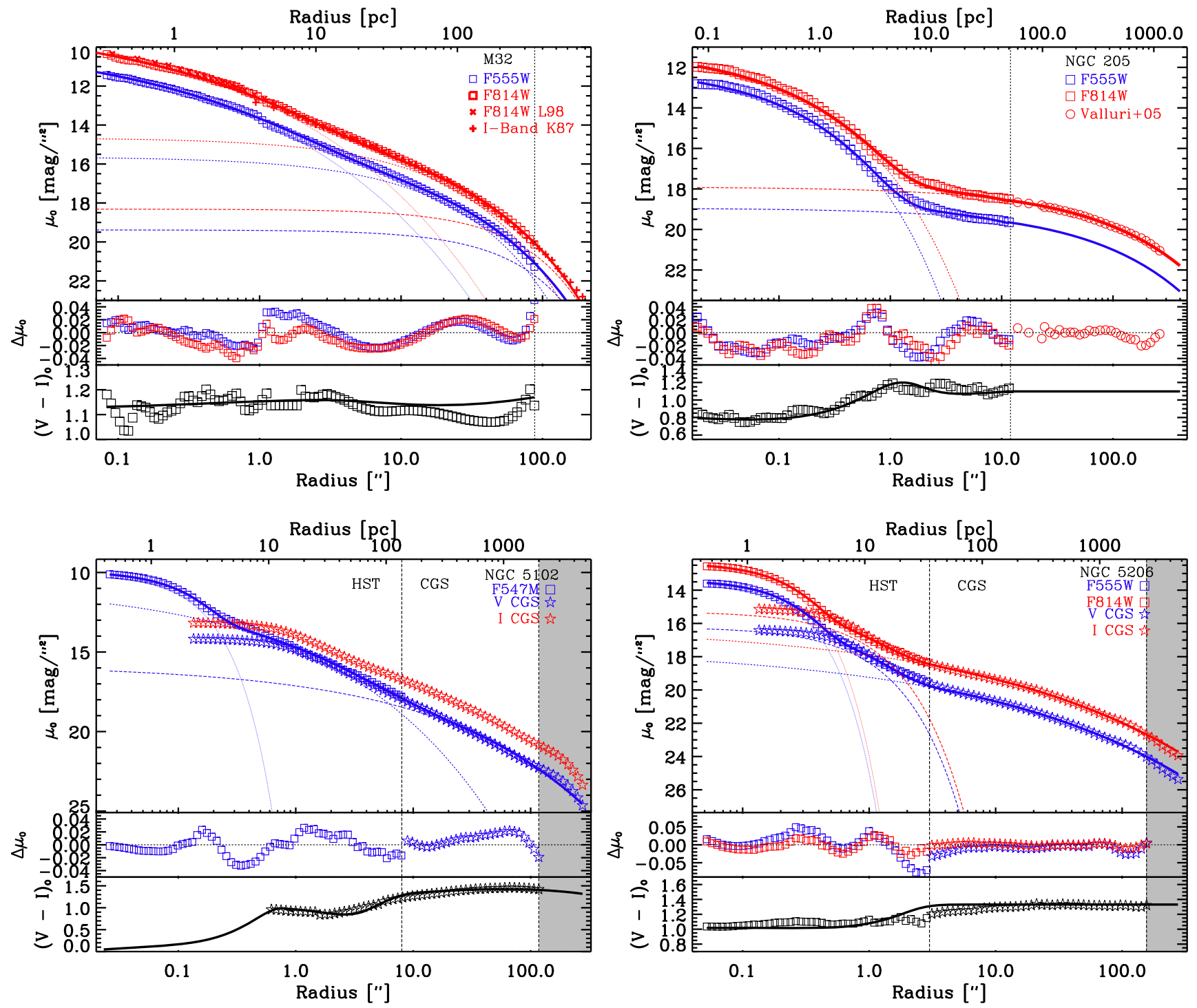

Figure 1. The SB profiles of each galaxy studied here. The profiles are constructed from a combination of ground-based and HST imaging. The blue lines show $V / F 555 \mathrm{~W} / \mathrm{F} 547 \mathrm{M}$ profiles, while the red lines show I/F814W profiles. All magnitudes/colors are corrected for foreground extinction. Symbols show the data, while the best-fitting 1D SB profiles are shown as solid lines. Each component of the best-fit models is also plotted for visualization (the innermost Sérsic component is shown as a dotted line, the second largest is shown as a dashed line, and, when present, the outermost component is shown as a long-dashed line). The residuals of the fits are shown in the middle panels, and the $(V-I)_{o}$ color profiles are illustrated in the bottom panels, including the data (black symbols) and the best-fit model (black line). Different symbols are plotted correspondingly to their data sets, which are shown in the legend of each top panel. The inner and outer vertical dashed lines show the ends of the HST and ground-based data, respectively. The gray regions in the plots of NGC 5102 and NGC 5206 indicate the areas beyond our fitting radius.

believe this is at least in part due to their normalization in the SB profile, which is nearly a magnitude higher than that derived here.

Next, we use the final fixed-PA 2D GALFIT Sérsic models to create multi-Gaussian expansion (MGE; Emsellem et al. 1994a, 1994b; Cappellari \& Emsellem 2004) models. These MGEs are comprised of a total of 18,10,14, and 14 Gaussians to provide a satisfactory fit to the surface mass density profiles of M32, NGC 205, NGC 5102, and NGC 5206, respectively. We fit our MGEs out to $\sim 110$ " 0 for M32 and NGC 5206, 15!"0 for NGC 205, and 21!"0 for NGC 5102 and obtain the mass models by straightforward multiplying the $M / L$ profiles with the 2D light GALFIT MGEs at the corresponding radii. We choose to parameterize the mass models using GALFIT (as opposed to using the fit_sectors_mge code) because (1) it enables us to properly incorporate the complex HST PSF and (2) it enables simple separation of NSC and galaxy components.

\subsection{Color $-\mathrm{M} / \mathrm{L}$ Relations}

To turn our stellar luminosity profiles into stellar-mass profiles, we assume an $M / L$-color relation. There is a strong correlation between the color and the $M / L$ of a stellar population: we use two different color $-M / L$ relations, including the Bell et al. (2003; hereafter B03) and (R15) color $-M / L$ correlations, as well as models with constant $M / L$. This is similar to the method presented by Nguyen et al. (2017) for analysis of the NGC 404 nucleus. For our best-fit models, we use the R15 relation, which was found by Nguyen et al. (2017) to be within $1 \sigma$ of the color$M / L$ relation derived from stellar population fits to STIS data within the nucleus. The B03 and constant $M / L$ models are used to assess the systematic uncertainties in our mass models. The 

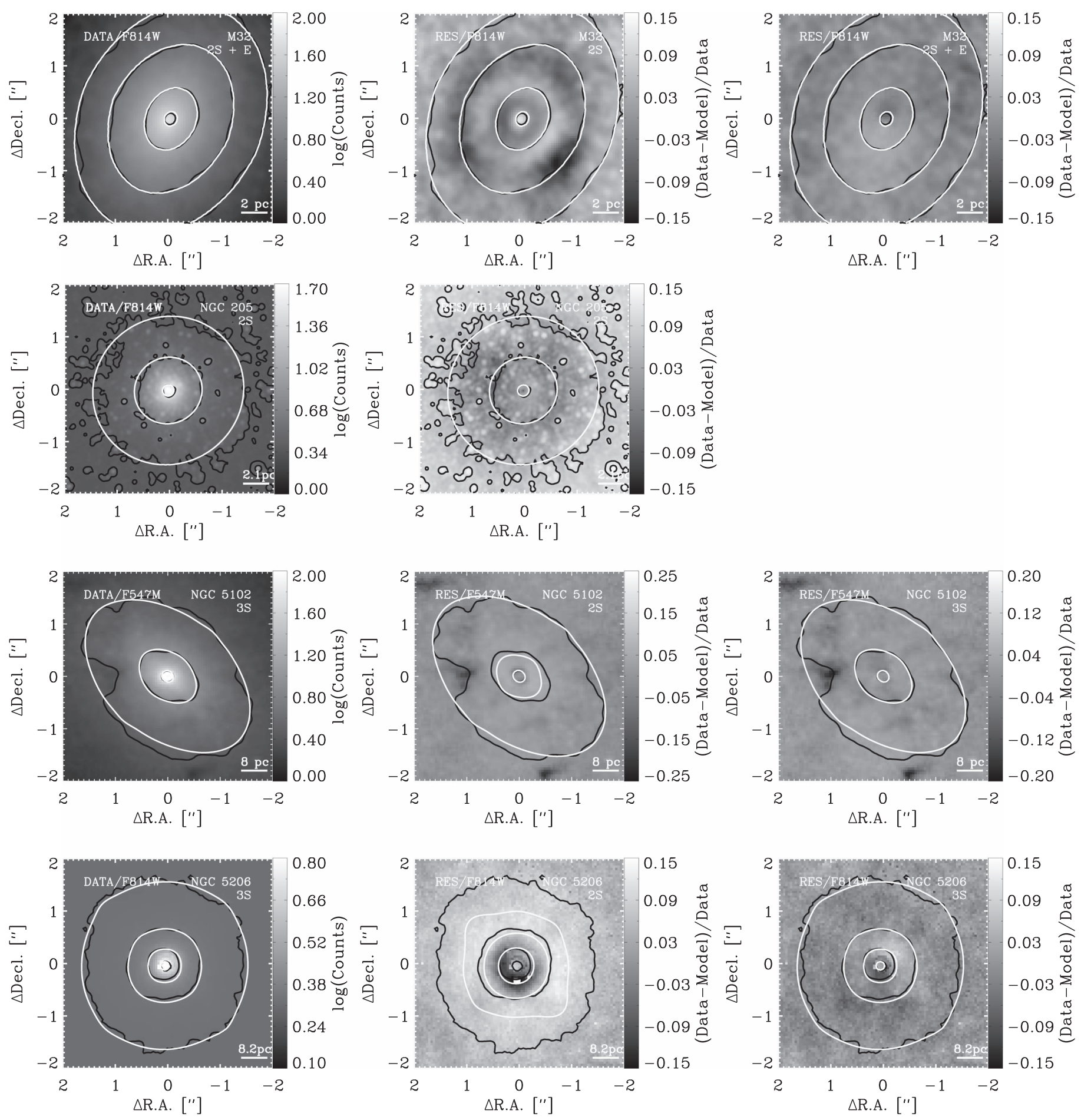

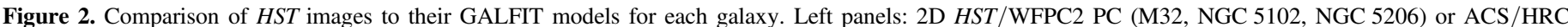

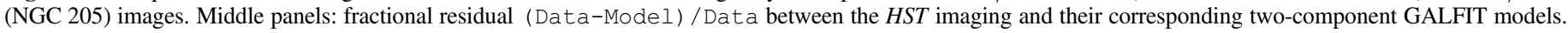

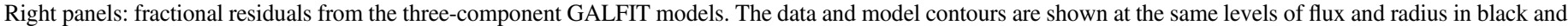
white repeating from the left to the right panels, respectively. All figures show east to the left and north up.

B03 and R15 relations are built based on the $(V-I)_{0}$ color profiles in the bottom panel of each plot in Figure 1, except in NGC 5102. For NGC 5102, we use the $(B-V)_{0}$ color estimated from the combined $H S T$ F547M-F656N data at small radii and F450W-F569W data at larger radii, as described in Appendix A.
We also create mass models with a constant central $M / L$. For these models, we take a reasonable reference value for the $M / L$ but still allow these values to be scaled in our dynamical models, just as for the varying $M / L$ models. We note that these models are not used in any of our final results but are used to analyze potential systematic errors in our dynamical modeling. 

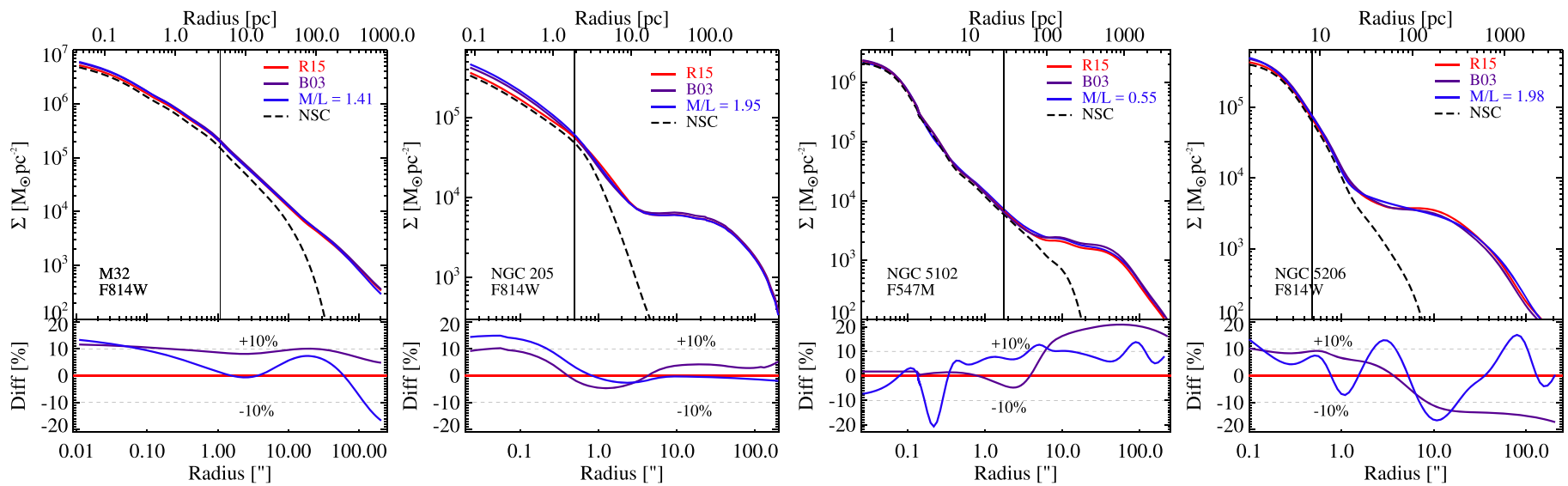

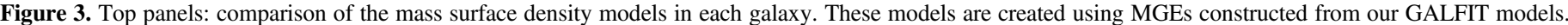

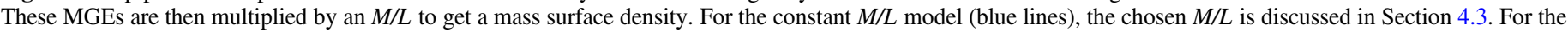

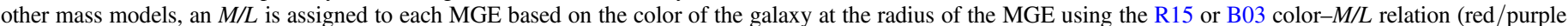

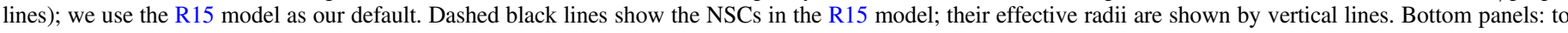

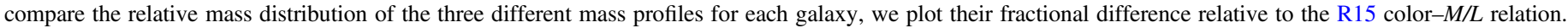

The reference values used for the constant $M / L$ models are as follows.

1. $M 32$. We use $M / L_{I}=1.41\left(M_{\odot} / L_{\odot}\right)$ based on the Schwarzschild model fits to Sauron data from Cappellari et al. (2006).

2. $N G C$ 205. We use a nucleus of $M / L_{I}=1.95\left(M_{\odot} / L_{\odot}\right)$, similar to that based on Schwarzschild model fits to STIS kinematics by Valluri et al. $2005\left(M / L_{I}=1.94\left(M_{\odot} / L_{\odot}\right)\right)$; note that this is the value found for the nucleus (they found a significantly higher $M / L$ for the galaxy as a whole).

3. $N G C$ 5102. We use $M / L_{V}=0.55\left(M_{\odot} / L_{\odot}\right)$ based on the stellar population model fits near the center of the galaxy from Figure 11 of Mitzkus et al. (2017) which uses the MILES simple stellar population library with a Salpeter IMF.

4. $N G C$ 5206. We use the photometric $M / L_{I}=1.98\left(M_{\odot} / L_{\odot}\right)$ based on the color $(V-I)_{o}=1.1$ and using estimates from Bell et al. (2003) and Bruzual \& Charlot (2003).

\subsection{Mass Models}

We create our final mass models for use in our dynamical modeling by multiplying the MGE luminosity created from the 2D GALFIT fit models of the images with PSF deconvolution (Section 4.2) with the $M / L$ profiles discussed in Section 4.3. We fit the MGE luminosity by using the sectors_photometry + mge_fit_sectors IDL $^{11}$ package. During these fits, we set their PAs and axial ratios of the Gaussians to be constants as their values obtained in GALFIT. More specifically, in NGC 5102, we calculate the $V$-band $M / L$ and apply this to the F547M data, while in the others, we calculate $I$-band $M / L$ s and apply these to the F814W data.

The top panel in each plot of Figure 3 shows the mass surface density, and the bottom panel shows the relative error of the $M / L$ profile relative to the $\mathrm{R} 15$ color $-M / L$ correlation for each galaxy. It is clear that the R 15 color $-M / L$ correlation (red line) predicts less mass at the center of each galaxy than the prediction of the B03 color- $M / L$ correlation (purple line). However, the assumption of constant $M / L$ s (blue line) predicts

\footnotetext{
${ }^{11}$ Available at http://purl.org/cappellari/software.
}

more mass at the centers but less mass at larger radii than the predictions of both $\mathrm{B} 03$ and $\mathrm{R} 15$ color $-M / L$ correlation. This is as expected based on the bluer centers and consequently lower $M / L$ s at the centers. The MGEs of mass surface densities of M32, NGC 205, NGC 5102, and NGC 5206 are presented in column (2) of Table 11 (Appendix B).

\subsection{K-band Luminosities}

Together with the stellar-mass distribution, the dynamical models also require as input the distribution of the stellar tracer population from which the kinematics are obtained. Given that the kinematics were derived in the $K$ band, which covers the wavelengths from 2.29 to $2.34 \mu \mathrm{m}$, we create high-resolution synthetic images in that band (Ahn et al. 2017). We do not use the $K$-band images obtained from the IFU observations directly, as they have lower resolution and too-limited FOV. To make these, we employ the Padova simple stellar population (SSP) model (Bressan et al. 2012) to fit color-color correlations. The purpose of this is to transform our HST imaging of F555W and F814W (of M32, NGC 205, and NGC 5206) into $K$-band images. Specifically, we fit the linear correlations of $H S T$ F555W-F814W versus $\mathrm{F} 814 \mathrm{~W}-K$ based on the specific nucleus stellar populations and metallicities for M32 ( $Z=0.019$; Corbin et al. 2001), NGC 205 ( $Z=0.008$; Butler \& Martínez-Delgado 2005), and NGC 5206 ( $Z=0.004-0.008$; N. Kacharov et al. 2018, in preparation). We use these to create $K$-band images based on the reference $H S T$ images (i.e., we add the color correction to the F814W image). These $K$-band images are similar to the $I$-band images, with deviations in the SB profile being comparable to differences between mass models shown in Figure 3.

In order to create a $K$-band image for NGC 5102, we use its F450W-F569W colors, which are inferred from the F547MF656N data as described in Appendix A. Next, we fit a linear correlation of HST F450W-F569W versus F569W-K using metallicities $Z=0.004$ (Davidge 2015) and then infer for its $K$-band images. We then fit the MGEs using the mge_fit_sectors IDL (see footnote 11) package. The MGEs $K$-band luminosity surface densities of M32, NGC 205, NGC 5102, and NGC 5206 are given in column (1) of Table 11 (Appendix B). 


\section{Stellar Kinematics Results}

We use adaptive optics NIFS and SINFONI spectroscopy to determine the nuclear stellar kinematics in all four galaxies. We first rebin spatial pixels within each wavelength of the data cube using the Voronoi binning method (Cappellari \& Copin 2003) to obtain $S / N \gtrsim 25$ per spectral pixel. Next, we use the pPXF method (Cappellari \& Emsellem 2004; Cappellari 2017) to derive the stellar kinematics from the $\mathrm{CO}$ band heads of the NIFS and SINFONI spectroscopy in the wavelength range of 2.280-2.395 $\mu \mathrm{m}$ and determine the line-of-sight velocity distribution (LOSVD). We fit only a Gaussian LOSVD, measuring the radial velocity $(V)$ and dispersion $(\sigma)$ using high spectral resolution of stellar templates of eight supergiant, giant, and main-sequence stars with spectral types between $\mathrm{G}$ and M with all luminosity classes (Wallace \& Hinkle 1996). These templates are matched to the resolution of the observations by convolving them by a Gaussian with dispersion equal to that of the line-spread function (LSF) of the observed spectra at every wavelength. These LSFs are determined from sky lines. For NIFS, the LSF is quite Gaussian with a FWHM $\sim 4.2 \AA$, but the width varies across the FOV (e.g., Seth 2010). However, the SINFONI LSF appears to be significantly non-Gaussian as seen from the shape of the $\mathrm{OH}$ sky lines. To characterize the shape of the LSF and its potential variation across the FOV, we reduced the sky frames in the same way as the science frames, with the difference that we did not subtract the sky. We then combined the reduced sky cubes using the same dither pattern as the science frames. This ensured that the measured LSF on the resulting sky lines fully resembles the one on the object lines. From these dithered sky lines, we measured the LSF. We used six isolated, strong sky lines, all having close doublets, except one (the $21995 \AA$ line), to measure the spectral resolution across the detector. Since the LSF appears to be constant along rows (constant $y$ values), the sky cubes were collapsed along the $y$ direction.

Sky emission $\mathrm{OH}$ lines can be described as a delta function, $\delta_{\lambda}$. Once they reach the spectrograph, they will be dispersed so that the intensity pattern is redistributed with wavelength according to the LSF of the instrument. Since we know the central wavelength of the sky emission lines, we assume that their shapes represent the LSF. Once located, the peak values in the spectra and a region around the peaks is defined, the continuum is subtracted, the line flux is normalized to the peak flux, and the lines are summed up.

The spectral resolution across the detector has a median value of $6.32 \AA \mathrm{FWHM}\left(\frac{\lambda}{\Delta \lambda}=3820\right)$, with values ranging from 5.46 to $6.7 \AA$ FWHM $(R \sim 3440-4300)$. The last step before the kinematic extraction is to perform the same binning on the LSF cube as for the science cube. This varying LSF is then used in the kinematic extraction with pPXF.

To obtain optimal kinematics from the SINFONI data, we had to correct for several effects: (1) a scattered-light component and (2) velocity differences between individual cubes. For the first issue, the SINFONI data cubes have imperfect sky subtraction, with clear additive residuals remaining despite subtraction of sky cubes. These residuals appear to have a uniform spectrum that is spatially constant across the field; however, the residuals are not clearly identified as sky or stellar spectra. To remove these residuals, we create a median residual spectrum for each individual data cube using pixels beyond 1". 3 radius and subtract it from all spaxels in the cube. This subtraction greatly improved the quality of our kinematic fits to our final combined data cube, but we note that this is likely also subtracting some galaxy light from each pixel. Second, fits to sky lines revealed variations in the wavelength solutions corresponding to velocity offsets of up to $20 \mathrm{~km} \mathrm{~s}^{-1}$ between individual data cubes. Therefore, before combining the cubes, we applied a velocity shift to correct these shifts; 4/12 cubes for NGC 5102 and 3/12 cubes for NGC 5206 were shifted before combining, bringing the radial velocity errors $\lesssim 2 \mathrm{~km} \mathrm{~s}^{-1}$ among the cubes to their means for both galaxies. We note, however, that applying these velocity shifts had minimal impact $\left(<0.5 \mathrm{~km} \mathrm{~s}^{-1}\right)$ on the derived dispersions. The systemic velocity in each galaxy was estimated by taking a median of pixels with radii $<0$ " 15 and is listed in Table 4.

To calculate the errors on the LOSVD, we add Gaussian random errors to each spectral pixel and apply Monte Carlo simulations to rerun the pPXF code. The errors used differ in NIFS and SINFONI; for NIFS, we have an error spectrum available, and these are used to run the Monte Carlo, while with SINFONI, no error spectrum is available, and we therefore use the standard deviation of the pPXF fit residuals as a uniform error on each pixel. We further test the robustness of our kinematic results by (1) fitting the spectra toward the short wavelength range $2.280-2.338 \mu \mathrm{m}$ of the CO band heads (the highest-S/N portion of the $\mathrm{CO}$ band head) and (2) using the PHOENIX model spectra (Husser et al. 2013), which have higher resolution $\left(R=500,000\right.$ or $\left.0.6 \mathrm{~km} \mathrm{~s}^{-1}\right)$ than the Wallace \& Hinkle $\left(1996 ; R=45,000\right.$ or $\left.6.67 \mathrm{~km} \mathrm{~s}^{-1}\right)$ templates. We find consistent kinematic results within the errors with item (1) returning dispersions $1-2 \mathrm{~km} \mathrm{~s}^{-1}$ higher than the full spectral range, while item (2) yields fully consistent results. We also verified our SINFONI kinematic errors by combining independent subsets of the data and identical binning and found that their distribution had a scatter similar to the error; a similar verification of the NIFS errors was done in Seth et al. (2014). This analysis indicates that the radial velocities and dispersion errors range from 0.5 to $20 \mathrm{~km} \mathrm{~s}^{-1}$. These kinematic data are presented in Tables 8-10 for NGC 205, NGC 5102, and NGC 5206, respectively, in Appendix B; M32 was published in Table 1 of Seth (2010).

Due to increased systematic errors affecting our kinematic measurements at low SB (where many pixels are binned together), we eliminate the outermost bins beyond an ellipse with semimajor axes of $1 ! 3,1 ! 2,0 ! 7$, and 0.77 in M32, NGC 5102, NGC 5206, and NGC 205. In general, we find that in the noisy data in outer regions, we overestimate our $V_{\mathrm{rms}}$ values. For instance, in the M32 data published in Seth (2010), we find a rise in $V_{\mathrm{rms}}$ beyond 1"'3; however, comparison with data from Verolme et al. (2002) suggests that this rise may be due to systematic error and is not used in the remainder of our analysis. In addition, we only plot and fit bins with dispersion and velocity errors $<15 \mathrm{~km} \mathrm{~s}^{-1}$. We correct the systemic velocities (then radial velocities) for the barycentric correction $\left(37,20,-20\right.$, and $-37 \mathrm{~km} \mathrm{~s}^{-1}$ for M32, NGC 205, NGC 5102, and NGC 5206). The systemic velocities are determined from the central velocity as references and double-checked using the fit_kinematic_pa IDL package (see footnote 11).

The final kinematic maps for NGC 205, NGC 5102, and NGC 5206 are shown in Figure 5. The left column shows the radial velocity maps, and the middle column shows the velocity dispersion maps. The right column shows the $V_{\mathrm{rms}}$ 

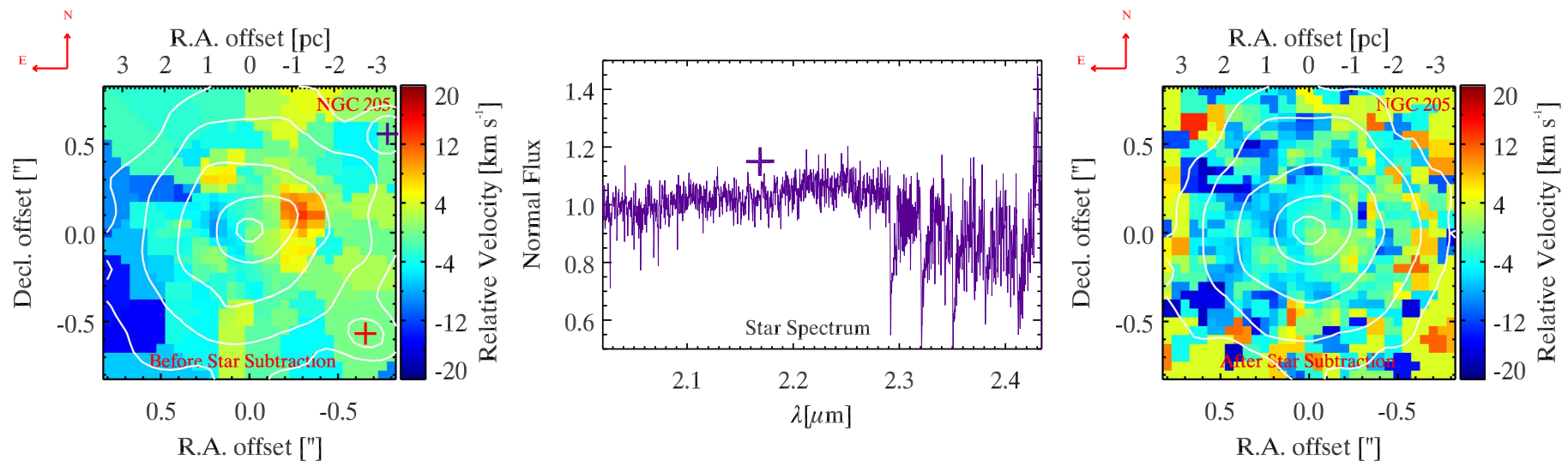

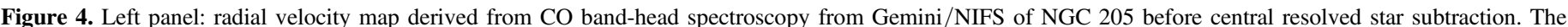

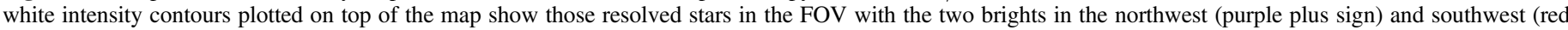

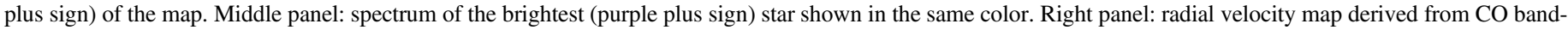

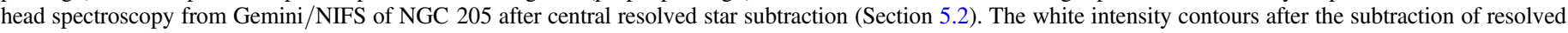
stars are now smoother.

measurements (gray diamonds with error bars, top panel) and the bi-weighted Vrms in the circular annuli (black circles with error bars). The bottom panel of the right column shows the results of KINEMETRY fits (Krajnović et al. 2006), which determine the best-fit rotation and dispersion profiles along ellipses. We discuss the kinematics for each galaxy below.

\subsection{M32}

The kinematics of M32 were derived with pPXF using a four-order of Gauss-Hermite series including $V, \sigma$, skewness $\left(h_{3}\right)$, and kurtosis $\left(h_{4}\right)$ (Seth 2010). These measurements are the highest-quality kinematic data available that resolve the sphere of influence (SOI) of the $\mathrm{BH}$. These kinematics show a very clear signature of a rotating and disky elliptical galaxy with strong rotation at a PA of $-25^{\circ}$ (E of $N$ ) with an amplitude of $\sim 55 \mathrm{~km} \mathrm{~s}^{-1}$ beyond the radius of 0 " 3 with the maximum of $V / \sigma$ reaching 0.76 at $r=0 . / 5(2 \mathrm{pc})$. The dispersion has a peak of $\sim 120 \mathrm{~km} \mathrm{~s}^{-1}$, which we will show is due to the influence of the $\mathrm{BH}$, and flattens out at $\sim 76 \mathrm{~km} \mathrm{~s}^{-1}$ beyond 0 ." 5 .

\subsection{NGC 205}

Of the nuclei considered here, NGC 205 is by far the one most dominated by individual stars. These are clearly visible in the HST images (Figure 2), and their effects can be seen in our kinematics maps. These maps show bright individual stars that often have decreased dispersion and larger offsets from the systemic velocity, and therefore we have attempted to remove them before deriving the kinematics, as done in Kamann et al. (2013). The spectrum of the star weighted by the PSF was subtracted from all adjacent spectra of the cube. We summarize this process briefly here. First, we identify bright stars in the FOV of NIFS data manually by eye and note their approximate spaxel coordinates in all layers of the data cube; there are 32 of these identified bright stars in total. Second, we estimate PSFs for these stars that are well-described analytically either by Moffat or double Gaussian; the differences between these two PSF representations are small, but we use the Moffat profile in our final analysis. Next, we perform a combined PSF fit for all identified stars; in this analysis, we model the contribution from the galaxy core as an additional component that is smoothly varying spatially. Third, we obtain spectra for all identified stars so that their information is used to subtract from each layer of the cube. Finally, we use this star-subtracted cube to determine the kinematics of the underlying background light of the galaxy.

We show our original map of relative velocity in the left panel of Figure 4. One of the subtracted stellar spectra is shown in the middle panel, while velocity map after the stellar subtraction is shown in the right panel. Subtraction of stars yields a much smoother velocity map and isophotal contours than our original map. The complete kinematic maps determined after star subtraction are shown in the top row of Figure 5. We use this map for all further analysis but also include the original map in tests of the robustness of our results.

We examine the stellar rotational velocity $(V)$ and dispersion velocity $\left(\sigma_{\star}\right)$ of NGC 205 . The dispersion velocity drops to $\sim 15 \mathrm{~km} \mathrm{~s}^{-1}$ within the 0 .' 2 radius and reaches $\sim 23 \mathrm{~km} \mathrm{~s}^{-1}$ in the outer annulus of 0 ". $2-0$ ". 8 . This dispersion velocity map is consistent with the radial dispersion profile obtained from HST/STIS data of the NGC 205 nucleus (Valluri et al. 2005) at the radius $>0$ ". 2 , although we find a slightly lower dispersion at the very nucleus than Valluri et al. (2005; $\Delta \sigma \sim 3 \mathrm{~km} \mathrm{~s}^{-1}$ ). This difference in dispersions is $\sim 1 \mathrm{~km} \mathrm{~s}^{-1}$ higher than the velocity dispersion errors of the central spectral bins (Table 8 in Appendix B). However, we caution that both sets of kinematics are close to the spectral resolution limits of the data. However, with the high $\mathrm{S} / \mathrm{N}$ of the central bins, and using our careful LSF determination, we believe we can reliably recover dispersions even at $\sim 15 \mathrm{~km} \mathrm{~s}^{-1}$ (Cappellari 2017).

The $V_{\mathrm{rms}}=\sqrt{V^{2}+\sigma^{2}}$ profile has a value of $20 \pm$ $5 \mathrm{~km} \mathrm{~s}^{-1}$ out to 0.7 . The maximum $V / \sigma \sim 0.33$ occurs at 0 ". 6 (2.6 pc), and this low level of rotation is seen throughout the cluster.

\subsection{NGC 5102}

The radial velocity map of the NGC 5102 nucleus shows clear rotation. This rotation reaches an amplitude of $\sim 30 \mathrm{~km} \mathrm{~s}^{-1}$ at 0 ." 6 from the nucleus. Beyond this radius, the rotation curve is flat out to the edge of our data. The dispersion is fairly flat at $\sim 42 \mathrm{~km} \mathrm{~s}^{-1}$ from $r=0$ ". 3 to 1 ". 2 with a peak at the center reaching $59 \pm 1 \mathrm{~km} \mathrm{~s}^{-1}$. The maximum $V / \sigma$ is $\sim 0.7$ at 0 ". 6 . This suggests that the second Sérsic component, which is identified as part of the NSC and is the most flattened component, may be strongly rotating. This component's 


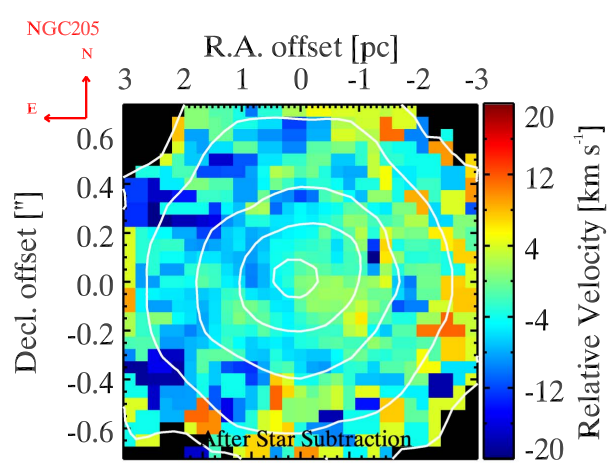

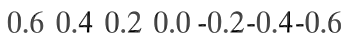

R.A. offset ["]
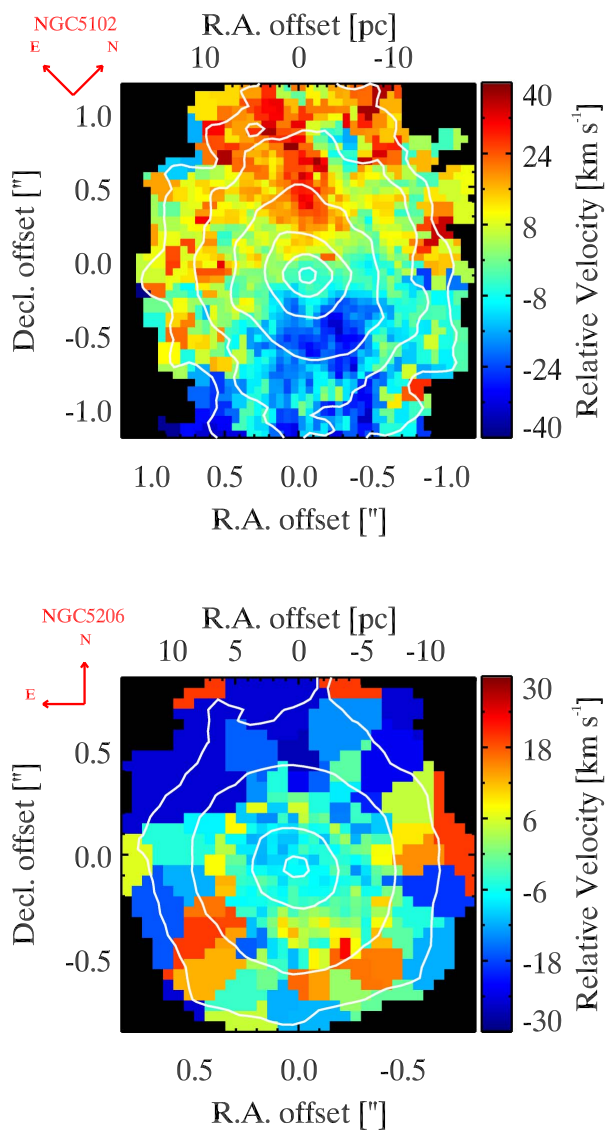

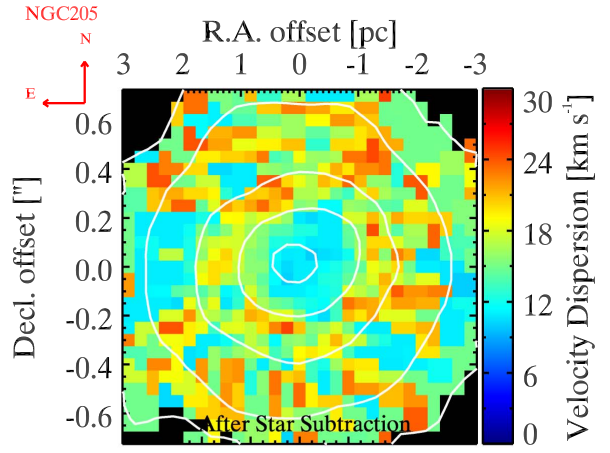

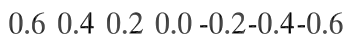

R.A. offset ["]
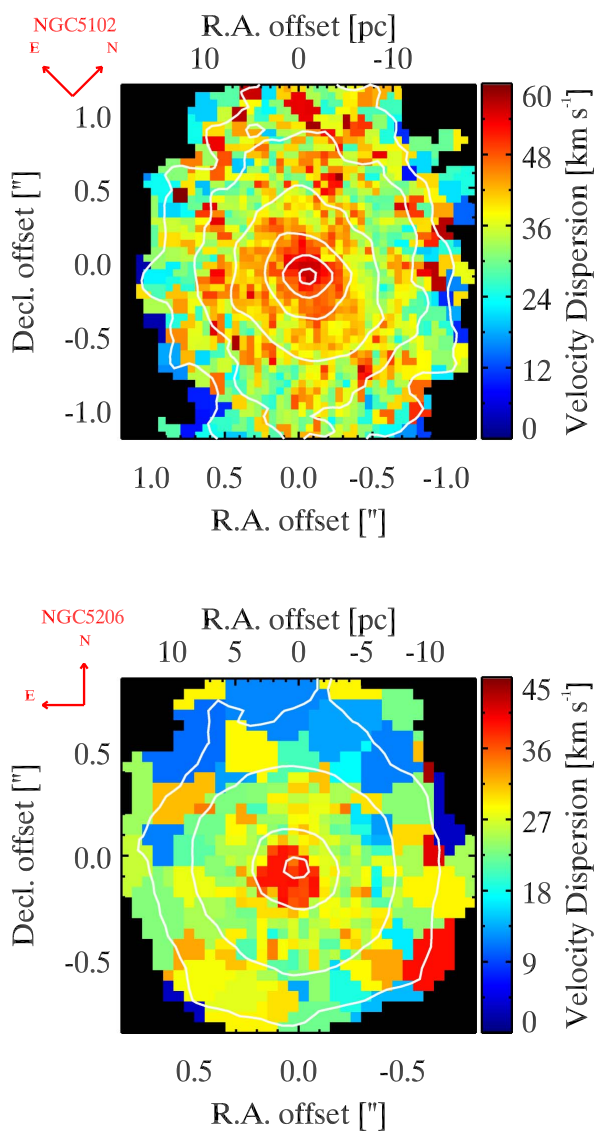

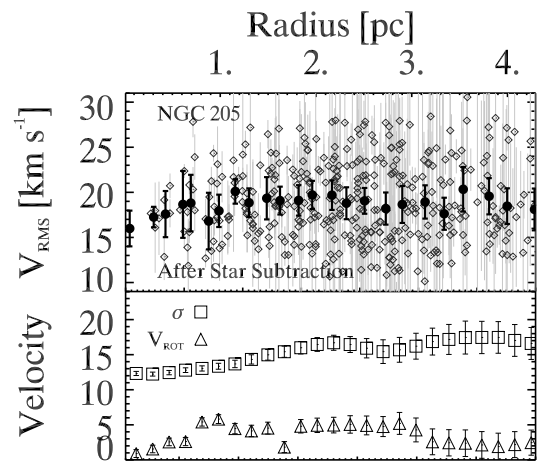

$\begin{array}{lllllll}0.1 & 0.2 & 0.3 & 0.4 & 0.5 & 0.6 & 0.7\end{array}$ Radius ["]
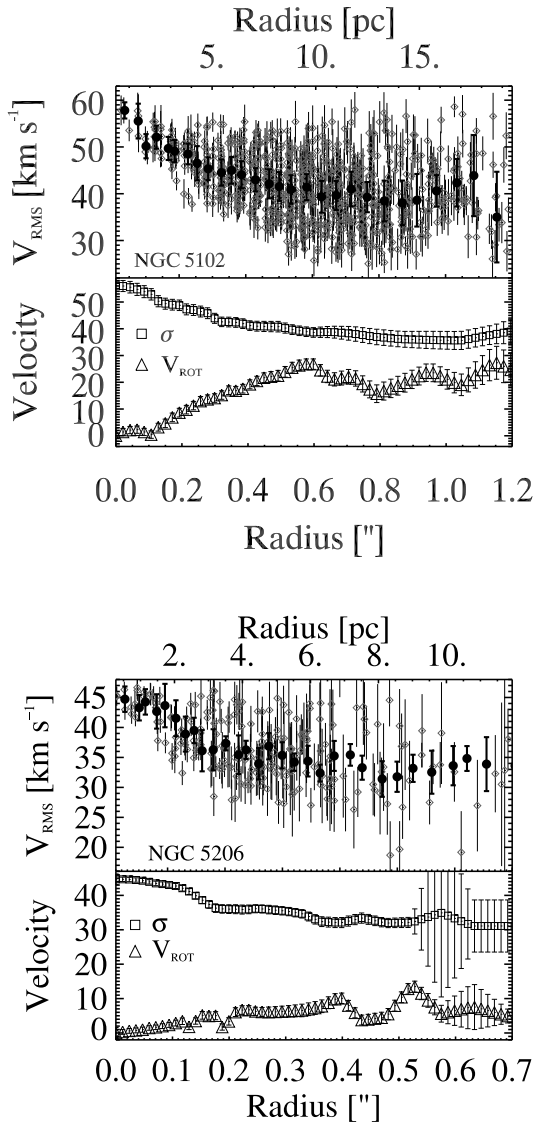

Figure 5. Stellar kinematic maps derived from CO band-head spectroscopy from Gemini/NIFS (NGC 205) and VLT/SINFONI (NGC 5102 and NGC 5206) spectroscopic data. Radial velocity maps are shown in the left column and dispersion maps in the middle column. The radial velocity is shown relative to the systemic velocity of $-241 \pm 2 \mathrm{~km} \mathrm{~s}^{-1}$ (NGC 205), $472 \pm 2 \mathrm{~km} \mathrm{~s}^{-1}$ (NGC 5102), and $573 \pm 5 \mathrm{~km} \mathrm{~s}^{-1}$ (NGC 5206). The top plots show the NGC 205 stellar kinematics after removing individual bright stars (Section 5.2). White contours show the stellar continuum, and red arrows indicate the orientation. Kinematics are only plotted out to the radii where they are reliable; black pixels indicate data not used in the JAM modeling. In the right column, the top panel shows the $V_{\mathrm{rms}}=\sqrt{V^{2}+\sigma^{2}}$ (gray diamonds), with the black circles showing the bi-weighted $V_{\text {rms }}$ in the circular annuli. The error bars are $1 \sigma$ deviations of the kinematic measurements of the Voronoi bins in the same annuli. The KINEMETRY decomposition (Krajnović et al. 2006) of the dispersion (squares) and rotation (triangles) curves is shown in the bottom panel.

position in the $V / \sigma$ versus $\epsilon(\epsilon=0.4$ and $V / \sigma=0.7)$ is consistent with a rotationally flattened system, especially given the best-fit inclination of $\sim 71^{\circ}$ we derive below.

Our kinematics are consistent with those from Mitzkus et al. (2017), who used MUSE spectroscopy to measure kinematics out to radii of $\sim 30^{\prime \prime}$. On larger scales, they found that the galaxy has two counterrotating stellar disks, with maximum rotation amplitudes of $\sim 20 \mathrm{~km} \mathrm{~s}^{-1}$ and dispersions very similar to our measured dispersion throughout the central $\sim 10^{\prime \prime}$.

\subsection{NGC 5206}

The radial velocity map of NGC 520 shows a small but significant rotation signal in the nucleus; this rotation appears to gradually rise outward with a maximum amplitude of 
Table 6

Gemini/NIFS and VLT/SINFONI Jeans Modeling Best-fit Results

\begin{tabular}{|c|c|c|c|c|c|c|c|c|c|c|c|}
\hline $\begin{array}{l}\text { Object } \\
\text { (1) }\end{array}$ & $\begin{array}{l}\text { Filter } \\
\text { (2) }\end{array}$ & $\begin{array}{c}\text { Color }-M / L \\
\text { (3) }\end{array}$ & $\begin{array}{c}M_{\mathrm{BH}} \\
\left(M_{\odot}\right) \\
(4)\end{array}$ & $\begin{array}{l}\beta_{\mathrm{z}} \\
(5)\end{array}$ & $\begin{array}{l}\gamma \\
(6)\end{array}$ & $\begin{array}{c}i \\
(\mathrm{deg}) \\
(7)\end{array}$ & $\begin{array}{l}\text { No. of Bins } \\
\text { (8) }\end{array}$ & $\begin{array}{l}\chi_{\mathrm{r}}^{2} \\
(9)\end{array}$ & $\begin{array}{c}\chi_{\mathrm{r}, \text { no BH }}^{2} \\
(10)\end{array}$ & $\begin{array}{c}r_{\mathrm{g}, \mathrm{dyn} .} \\
(\operatorname{arcsec}) \\
(11)\end{array}$ & $\begin{array}{l}r_{\mathrm{g}, \text { dyn. }} \\
(\mathrm{pc}) \\
(12)\end{array}$ \\
\hline M32 & $\mathrm{F} 814 \mathrm{~W}^{\mathrm{G}}$ & R15 & $2.5_{-1.0}^{+0.6} \times 10^{6}$ & $-0.20_{-0.35}^{+0.30}$ & $1.08_{-0.30}^{+0.20}$ & $70.0_{-6.0}^{+6.0}$ & 1354 & 1.12 & 6.72 & 0.404 & $\overline{1.61}$ \\
\hline NGC 205 & $\mathrm{~F} 814 \mathrm{~W}^{\mathrm{G}}$ & R15 & $2.5_{-2.5}^{+4.7} \times 10^{4}$ & $0.27_{-0.10}^{+0.07}$ & $1.10_{-0.18}^{+0.10}$ & $59.0_{-8.0}^{+5.0}$ & 256 & 1.24 & 1.20 & 0.033 & 0.14 \\
\hline NGC 5102 & $\mathrm{~F} 547 \mathrm{M}^{\mathrm{G}}$ & R15 & $8.8_{-6.6}^{+4.2} \times 10^{5}$ & $0.15_{-0.16}^{+0.16}$ & $1.15_{-0.03}^{+0.06}$ & $71.5_{-4.0}^{+9.0}$ & 1017 & 1.12 & 3.57 & 0.070 & 1.20 \\
\hline NGC 5206 & $\mathrm{~F} 814 \mathrm{~W}^{\mathrm{G}}$ & R15 & $4.7_{-3.4}^{+2.3} \times 10^{5}$ & $0.25_{-0.12}^{+0.08}$ & $1.06_{-0.05}^{+0.05}$ & $44.0_{-5.0}^{+8.5}$ & 240 & 1.20 & 2.69 & 0.058 & 1.00 \\
\hline
\end{tabular}

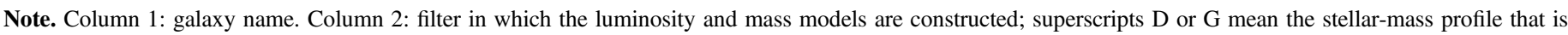

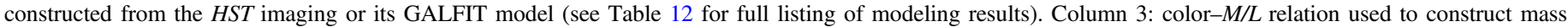

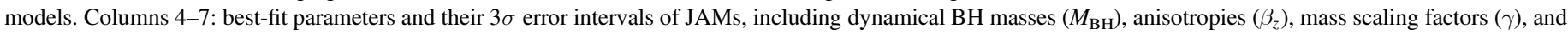

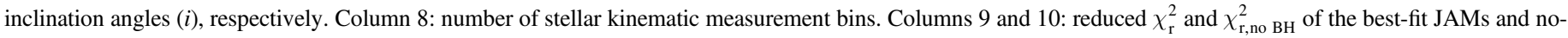
BH cases, respectively. Columns 11 and 12: best-fit BH SOI in arcsec and pc.

$10-15 \mathrm{~km} \mathrm{~s}^{-1}$ at 0 ". 5 . Our decomposition of the nucleus shows a nearly round system $(b / a=0.96)$ for both NSC components; however, the maximum $V / \sigma_{\star} \sim 0.3$ at $0 . " 5$ suggests that the outer of these two components may be more strongly rotating. Similar to NGC 5102, the NGC 5206 dispersion map shows a fairly flat dispersion of $33 \mathrm{~km} \mathrm{~s}^{-1}$ from $r=0$ !" 4 to 0 !' 7 , increasing to $46 \mathrm{~km} \mathrm{~s}^{-1}$ at the center.

\section{Stellar Dynamical Modeling and BH Mass Estimates}

In this section, we fit the stellar kinematics using JAMs and present estimates of the $\mathrm{BH}$ masses.

\subsection{Jeans Anisotropic Models}

We create dynamical models using the JAM of Cappellari (2008). ${ }^{12}$ Given the strong gradients in the stellar population of some of the galaxies, the mass-follows-light assumption is not generally acceptable. For this reason, we distinguish the parameterization of the stellar mass from that of the tracer population. This approach was also used for the same reason in the JAMs by Ahn et al. (2017), Li et al. (2017), Mitzkus et al. (2017), Nguyen et al. (2017), Poci et al. (2017), and Thater et al. (2017). Specifically, we used the MGEs derived using the default R15 color relation to parameterize the stellar mass, but we adopted the $K$-band luminosity MGEs to parameterize the tracer population in the JAM. We use the JAM to predict the second velocity moment, $V_{\mathrm{rms}}=\sqrt{V^{2}+\sigma^{2}}$, where $V$ is the radial velocity relative to the systemic velocity and $\sigma$ is the LOS velocity dispersion (Section 5). The JAMs have four free parameters- $\mathrm{BH}$ mass $\left(M_{\mathrm{BH}}\right)$, mass scaling factor $\left(\gamma=\left(M / L_{\text {dyn. }}\right) /\left(M / L_{\text {pop. }}\right)\right)$ assuming Salpeter IMF, anisotropy $\left(\beta_{z}\right)$, and inclination angle $(i)$ - that relate the gravitational potential to the second velocity moments of the stellar velocity distribution. These second velocity moment predictions are projected into the observational space to predict the $V_{\text {rms }}$ in each kinematic bin using the luminosity model (synthetic $K$-band luminosity) and kinematic PSF. The anisotropy parameter $\left(\beta_{z}\right)$ relates the velocity dispersion in the radial direction $\left(\sigma_{R}\right)$ and $z$-direction $\left(\sigma_{z}\right): \beta_{z}=1-\sigma_{z}^{2} / \sigma_{R}^{2}$, assuming the velocity ellipsoid is aligned with cylindrical coordinates. The predicted $V_{\mathrm{rms}}$ is compared to the observations, and a $\chi^{2}$ is determined for each model. The number of kinematic bins for the four galaxies is shown in Column 8 of Table 6 . To find the best-fit parameters, we construct a grid of values for the four parameters $\left(\beta_{z}, \gamma, M_{\mathrm{BH}}, i\right)$.

\footnotetext{
${ }^{12}$ Specifically, we use the IDL version of the code, available at http://purl. org/cappellari/software.
}

For each triplet, parameters of $\left(\beta_{z}, M_{\mathrm{BH}}, i\right)$, we linearly scale the $\gamma$ parameter to match the data in a $\chi^{2}$ evaluation. We run coarse grids in our parameters to isolate the regions with acceptable models and then run finer grids over those regions, as shown in Figure 6.

Figure 6 shows $\chi^{2}$ contours as a function of $M_{\mathrm{BH}}$ versus $\beta_{z}$ (left), $M_{\mathrm{BH}}$ versus $\gamma$ (middle), and $M_{\mathrm{BH}}$ versus $i$ (right). The best-fit $\mathrm{BH}$ masses are shown by red dots with three contours showing the $1 \sigma, 2 \sigma$ (thin red lines), and $3 \sigma$ (thick red lines) levels or $\Delta \chi^{2}=2.30(\sim 68 \%), 6.18(\sim 97 \%)$, and 11.81 $(\sim 99.7 \%)$ after marginalizing over the other two parameters. The best-fit $M_{\mathrm{BH}}, \beta_{z}, \gamma$, and $i$ and minimum reduced $\chi^{2}$ are shown Table 6 . We quote our uncertainties below on the $\mathrm{BH}$ mass and other parameters at the $3 \sigma$ level. Given the restrictions JAMs place on the orbital freedom of the system (relative to, e.g., Schwarzschild models), it is common to use $3 \sigma$ limits/ detections in quoting BH masses (see Seth et al. 2014 and Section 4.3.1 of Nguyen et al. 2017 for additional discussion).

\subsubsection{M32}

The best-fit JAM of M32 gives $M_{\mathrm{BH}}=2.5_{-1.0}^{+0.6} \times 10^{6} M_{\odot}$, $\beta_{z}=-0.20_{-0.35}^{+0.30}, \gamma=1.08_{-0.30}^{+0.20}$, and $i=70_{-6}^{\circ+6}$. We note that the wide range in uncertainty in $\beta_{z}$ is likely due to the bulk of our kinematic data lying within the SOI of the BH. Our BH mass, $M / L$, and $i$ estimates are fully consistent with values presented in Verolme et al. (2002), Cappellari et al. (2006), and van den Bosch \& de Zeeuw (2010) after correcting to a common distance of $0.79 \mathrm{Mpc}$ and for extinction $A_{B}=0.177 \mathrm{mag}$ (Schlafly \& Finkbeiner 2011) and assuming $A_{I}=A_{B} / 2.22$. The consistency of the BH masses in M32 shows the good agreement between JAM and Schwarzschild modeling techniques (see Section 6.2.3).

\subsubsection{NGC 205}

The JAMs of NGC 205 are compared to the kinematics derived after subtracting bright stars in the nucleus (see Section 5.2). The data are consistent with no $\mathrm{BH}$ even at the $1 \sigma$ level, and we find that a $3 \sigma$ upper limit would be $M_{\mathrm{BH}}$ $=7 \times 10^{4} M_{\odot}$. The $\beta_{z}, \gamma$, and $i$ have a range of values of $(0.17-0.34),(0.97-1.22)$, and $\left(51^{\circ}-65^{\circ}\right)$, respectively. We note that if we use the original kinematics (without star subtraction), we get a similar BH mass, inclination, anisotropy, and $\gamma$ values, with a slightly higher $3 \sigma \mathrm{BH}$ mass upper limit of $10^{5} \mathrm{M}_{\odot}$. Our best-fit BH mass for NGC 205 is twice the $3 \sigma$ upper mass limit of Valluri et al. (2005), although we find the same $M / L_{I}$. 

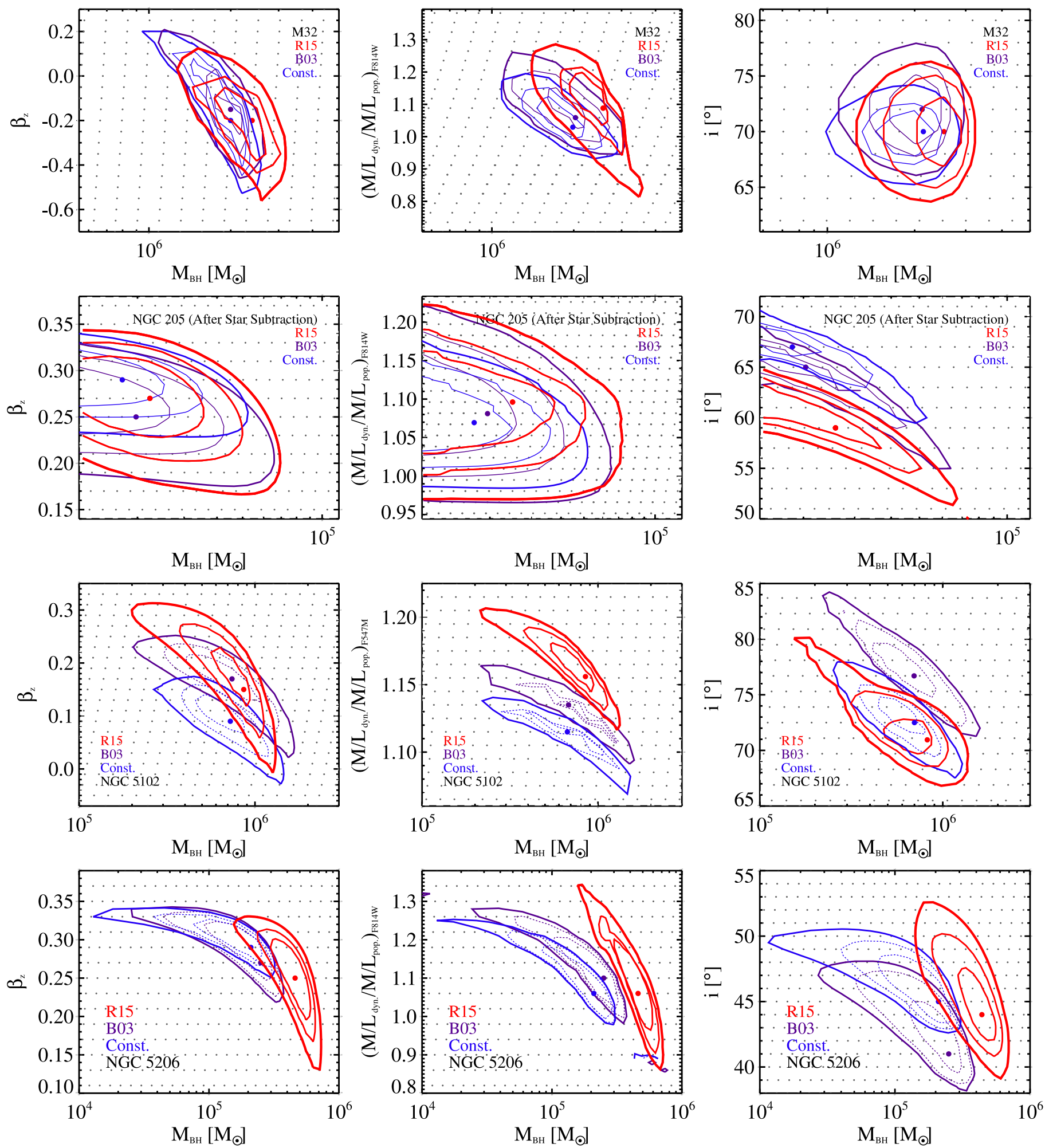

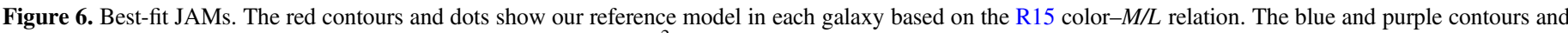

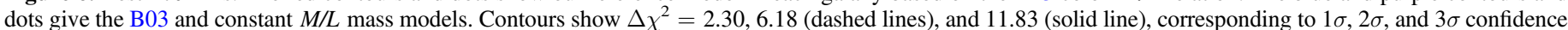

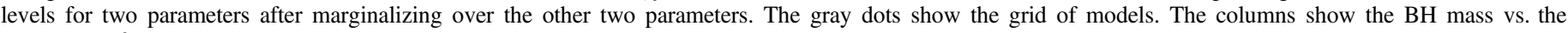
anisotropy, $\beta_{z}$ (left); mass scaling factor, $\gamma=M / L_{\text {dyn. }} / M / L_{\text {pop. }}$ (center); and inclination, $i$ (right).

\subsubsection{NGC 5102 and NGC 5206}

A BH of NGC 5102 is detected with a zero-mass BH excluded at more than the $3 \sigma$ level. The BH of NGC 5206 is also detected here at the $3 \sigma$ level in the default R 15 models, but the B03 and constant $M / L$ models are consistent with zero mass at the $\gtrsim 4 \sigma$ level. We emphasize here that this is the first time these BHs are clearly detected and these masses are measured in the sub-million $M_{\odot}$ regime with the properties listed in Table 6. 

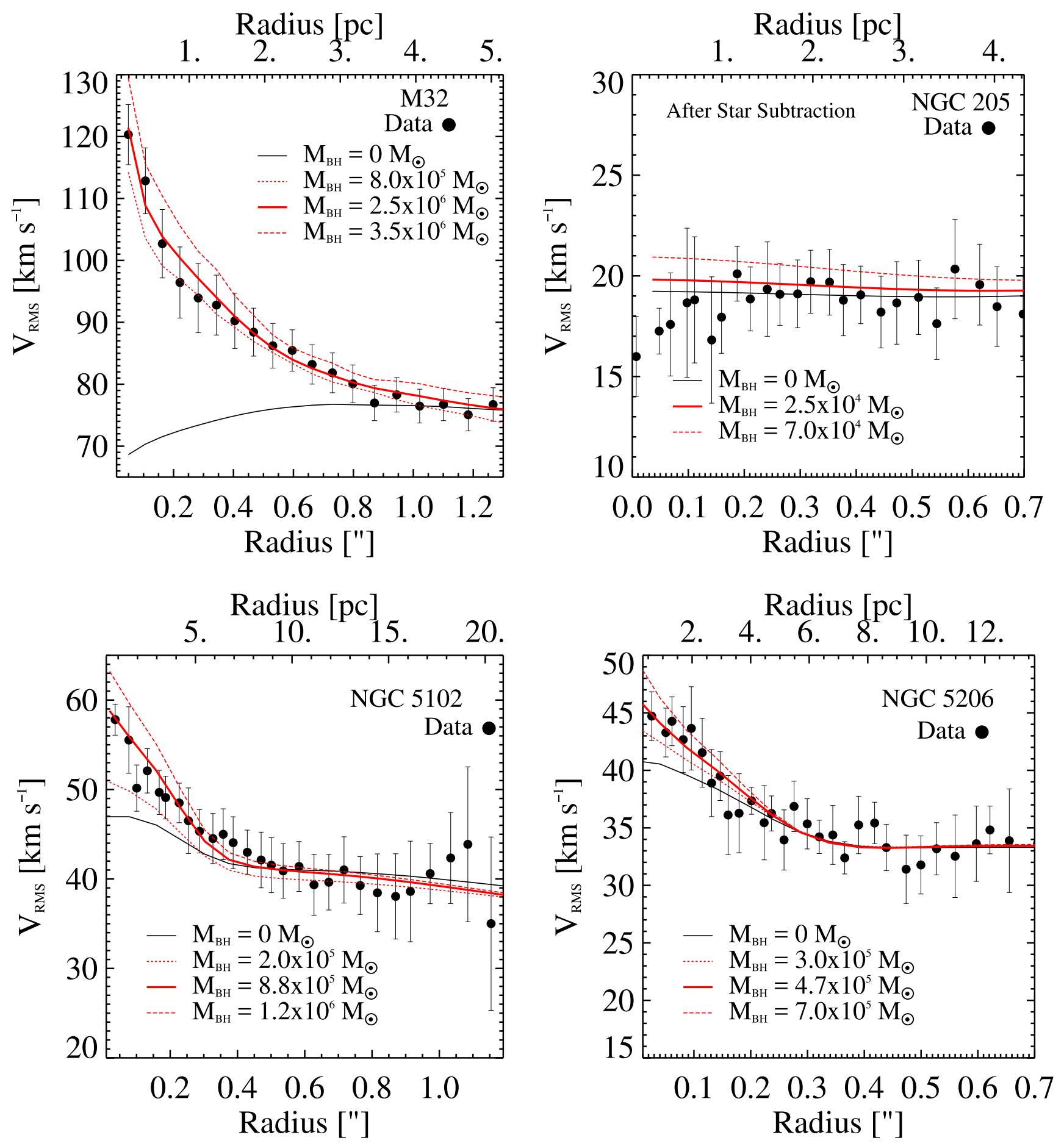

Figure 7. The 1D $V_{\text {rms }}$ vs. JAM predictions of mass models with varying BH masses. The red solid lines show our best-fit JAMs. The long-dashed and short-dashed lines indicate the upper and lower range of BH masses within $3 \sigma$ (see Figure 6 and Table 6), while the black lines show the best-fit JAMs without a BH. All models are fixed to the corresponding best-fit inclination angles of galaxies (column 9, Table 6) but varying for anisotropy, mass scaling ratio, and BH mass, which will be written in the form of $\left(\beta_{z}, \gamma, M_{\mathrm{BH}}, i\right)$. The specific zero-mass and best-fit $\mathrm{BH}$ models plotted have $\left(0.10,1.20,0 M_{\odot}, 70^{\circ}\right)$ and $\left(-0.2,1.08,2.5 \times 10^{6} M_{\odot}, 70^{\circ}\right)$ for $\mathrm{M} 32,(0.27$, $\left.1.12,0 M_{\odot}, 63^{\circ}\right)$ and $\left(0.27,1.10,7.0 \times 10^{4} M_{\odot}, 55^{\circ}\right)$ for NGC $205,\left(0.26,1.19,0 M_{\odot}, 71.5\right)$ and $\left(0.15,1.15,8.8 \times 10^{5} M_{\odot}, 71.5\right)$ for NGC 5102, and $(0.30,1.16$, $\left.0 M_{\odot}, 44.5\right)$ and $\left(0.25,1.06,4.7 \times 10^{5} M_{\odot}, 45^{\circ} .5\right)$ for NGC 5206. Both the $V_{\text {rms }}$ profiles of spectroscopic data and their corresponding JAM predictions are binned radially in the same manner for all galaxies as presented in the right panels of Figure 5. The error bars are $1 \sigma$ deviations of the kinematic measurements of the Voronoi bins in the same annuli.

To illustrate the best-fit JAMs with and without a BH, we plot the 1D and 2D $V_{\text {rms }}$ predicted by the JAM and compare them with their corresponding data in Figures 7 and 8, respectively. The $1 \mathrm{D} V_{\text {rms }}$ profiles show the bi-weighted average over circular annuli, as shown in the top right panel of Figure 5. Overplotted on these data are the best-fit models, those with the maximum and minimum $\mathrm{BH}$ mass in the $3 \sigma$ contours, and the best-fit no-BH model. These show that models with $M_{\mathrm{BH}}=0 M_{\odot}$ do not provide a good fit to the data, except for NGC 205. We quote both the corresponding $\chi_{\mathrm{r}}^{2}$ and $\chi_{\mathrm{r}, \mathrm{no} \text { BH }}^{2}$ for the best fit with and without a BH in Table 6. 

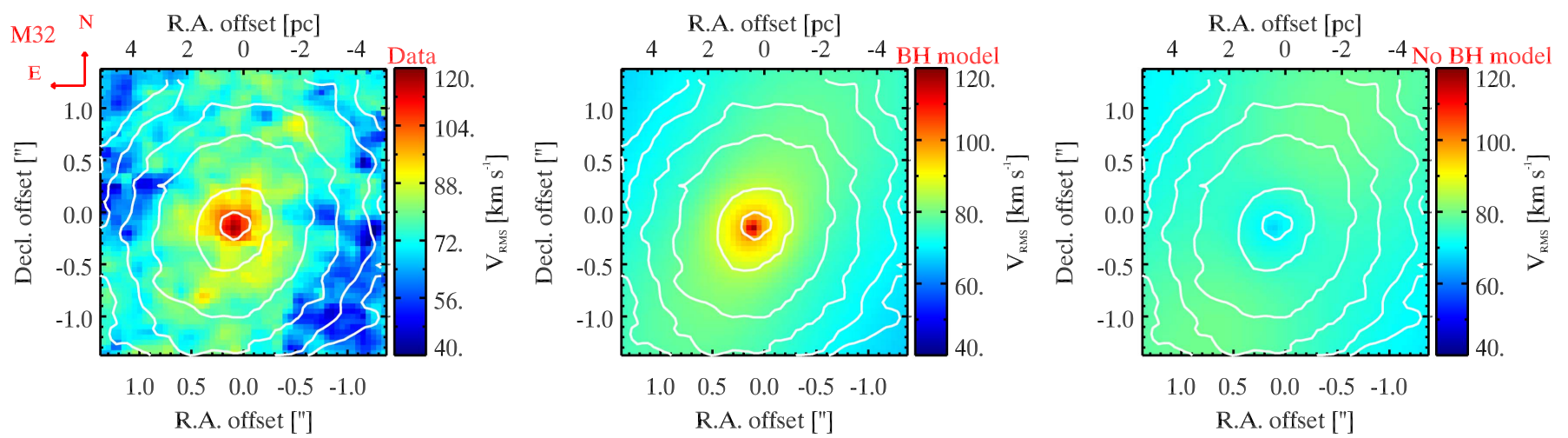

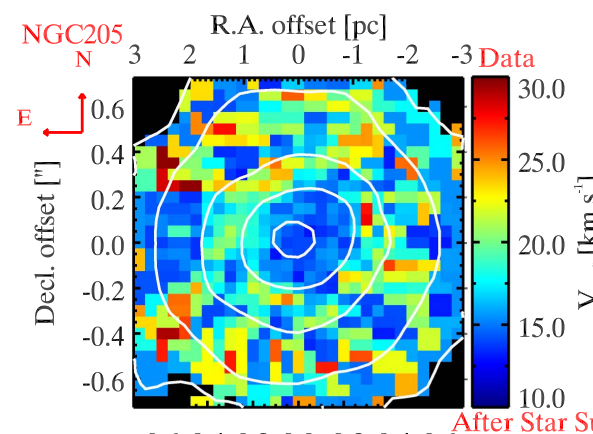

$0.60 .4 \quad 0.2 \quad 0.0-0.2-0.4-0.6$

R.A. offset ["]

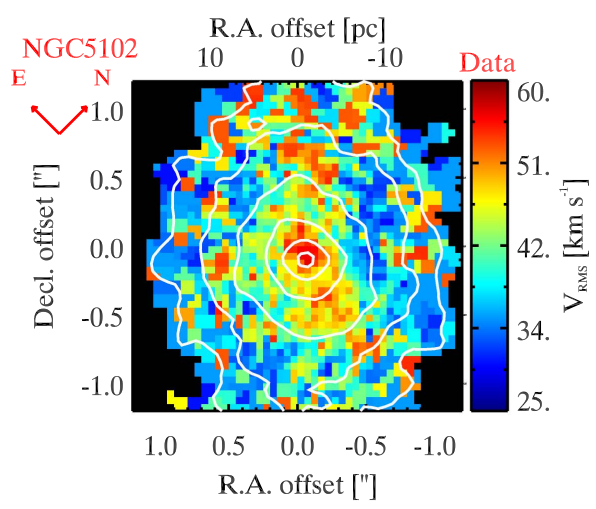

R.A. offset [pc]

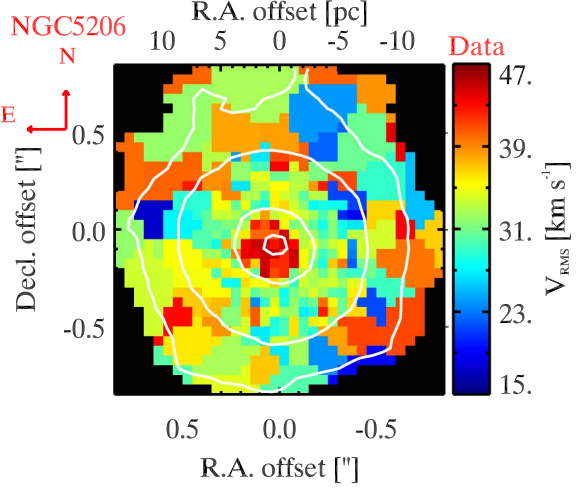

R.A. offset [pc]

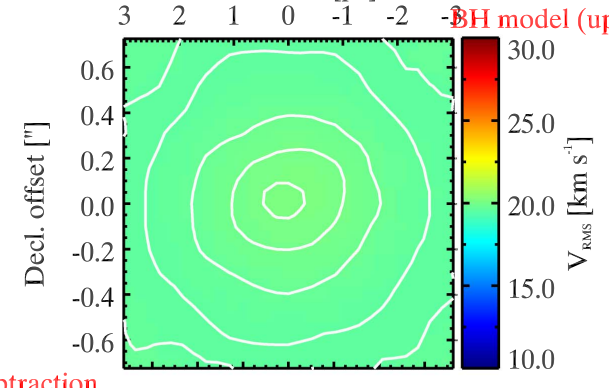

$0.60 .4 \quad 0.2 \quad 0.0-0.2-0.4-0.6$

R.A. offset ["]
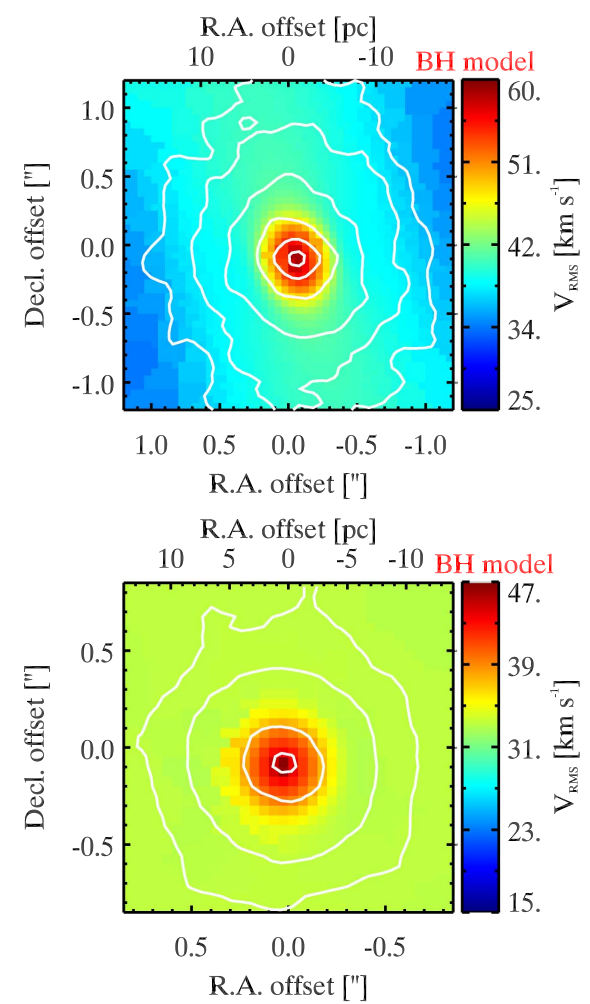

R.A. offset [pc]

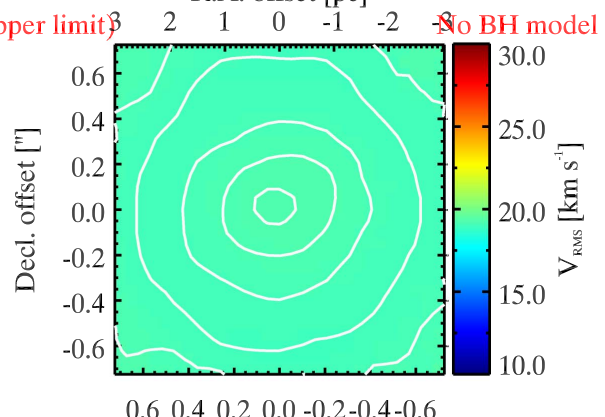

R.A. offset ["]
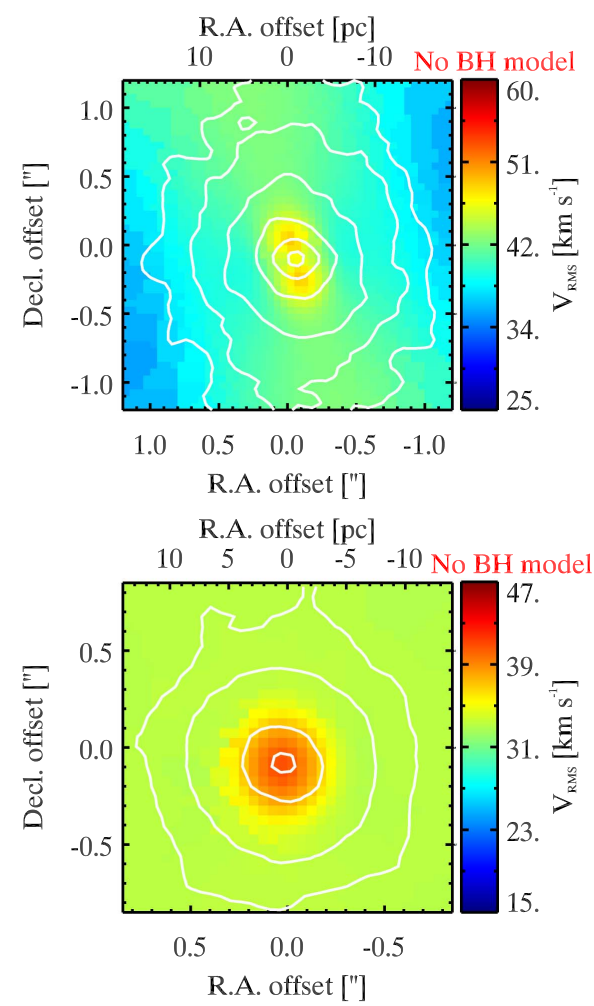

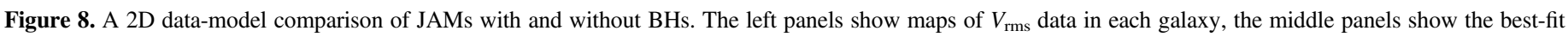

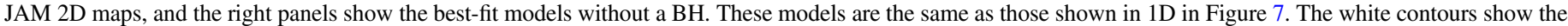
continuum, and the red arrows show the orientation.

The 1D profiles show that the radial data agree quite well with the models, with the only significant discrepancy being at the center of NGC 205. This may be due to mass-model issues in NGC 205 due to both the partially resolved kinematic data and the strong color gradient near the center. However, we also note that the dispersion measurements are very low, falling below the instrumental resolution of the telescope $\left(\sigma_{\mathrm{LSF}} \sim 23 \mathrm{~km} \mathrm{~s}^{-1}\right)$.

With the best-fit $\mathrm{BH}$ mass value, the SOI is calculated based on the dynamics: $r_{\mathrm{g}, \text { dyn. }}=G M_{\mathrm{BH}} / \sigma^{2}$, where $\sigma_{\star}$ is the stellar velocity dispersion of the bulge and $G$ is the gravitational constant. The calculations of $\sigma$ will be discussed in Section 8.2. 
Alternatively, we also estimate these BH SOIs via our mass profile, where $r_{\mathrm{g}, \star}$ is the radius at which the enclosed stellar mass is equal to $M_{\mathrm{BH}}$ (Merritt 2013). The results of $r_{\mathrm{g}, \text { dyn. of }}$ these BH SOIs are presented in Table 6 in both arcsec (column 10 ) and pc (column 11). Both methods give consistent values of the BH SOI $\left(r_{\mathrm{g}, \text { dyn. }} \simeq r_{\mathrm{g}, \star}\right)$. The $r_{\mathrm{g}, \text { dyn. }}$ of $\sim 0$ ". 07 and $\sim 0$ ". 06 in NGC 5102 and NGC 5206 are comparable to the FWHMs of the data, suggesting that the observational signatures of these $\mathrm{BHs}$ are just marginally resolved, while in $\mathrm{M} 32$, the $\mathrm{BH}$ signature is easily resolved by our PSF. For NGC 205, its $3 \sigma$ upper limit SOI $(\lesssim 0$ !"03) is comparable to the resolution of ACS HRC imaging.

\subsection{Sources of BH Mass Uncertainty}

In this section, we discuss possible sources of uncertainties in our dynamical models for $\mathrm{BH}$ mass measurements. The confidence intervals in our analysis are based on the kinematic measurement errors but do not include any systematic uncertainties in mass models, in the PSF (particularly for NGC 5206), and due to the JAM dynamical model that we used. We focus on these uncertainties here and show that for our $M_{\mathrm{BH}}$ estimates, these systematics are smaller than the quoted $3 \sigma$ confidence intervals.

\subsubsection{Uncertainties Due to Mass Models}

We examine the mass-model uncertainties by analyzing JAM results from using models (1) with constant $M / L$ or constructed using the B03 color $-M / L$ correlation, (2) based on different HST filters, and (3) fit directly to the HST imaging rather than based on the GALFIT model results. Our tests show that our $\mathrm{BH}$ mass results do not strongly depend on the mass models we use for M32, NGC 205, and NGC 5102, although in some cases, the best-fit $\gamma$ varies significantly because the centers of the galaxies are bluer than their surroundings. For NGC 5206, the constant $M / L$ or B03 models are basically consistent at the $3 \sigma$ level with no $\mathrm{BH}$, although the best-fit $\mathrm{BH}$ from this model is still within our $3 \sigma$ uncertainties. We note that the color gradient in the central $1^{\prime \prime}$ is quite modest, but the R15 model predicts $10 \%$ lower mass at the center than the other two mass models. The complete results of these mass-model tests are given in detail in Table 12.

(a) Existing color- $M / L$ models. We use the R15 color$M / L$ relation for our default mass models (Section 6.1). Here we examine how much our JAM modeling results would change if we use the shallower B03 color $-M / L$ relation or a constant $M / L$ to construct our mass models. Because our target galaxies are mostly bluer at their centers (except for M32), we expect that the use of a constant $M / L$ will reduce the significance of any $\mathrm{BH}$ component due to the increased stellar mass at the center relative to our reference R15 model. We show the best-fit JAM parameters using these alternative mass models in Figure 6, with the purple lines showing the B03 color $-M / L$ relation model and the blue lines showing a constant $M / L$ model, with dashed lines for $1 \sigma$ and $2 \sigma$ and solid lines for $3 \sigma$. As expected, these alternative models typically have lower BH masses than our default model, but in M32, NGC 205, and NGC 5102, the shifts are within $1 \sigma$ and do not significantly affect our interpretation. However, for NGC 5206, the shift is larger; the best fits are still within the R15 models' $3 \sigma$ uncertainties, but no $\mathrm{BH}$ models are permitted within $\sim 3 \sigma$ for both the constant $M / L$ and B03-based mass models. For
NGC 5102, the mass scaling $(\gamma)$ values differ significantly, with the best-fit constant $M / L$ model being more than $3 \sigma$ below the R15 model confidence intervals. Overall, these results are in agreement with those determined for NGC 404 (Nguyen et al. 2017).

(b) Mass models from other filters. We recreate our mass models based on HST images of other filters. Specifically, we use the $\mathrm{R} 15$ and $\mathrm{B} 03$ color $-M / L$ relations, as well as the constant $M / L$ on the F555W images for M32, NGC 205, and NGC 5206. We find that these mass models do not significantly change our results.

(c) Mass models from direct fits to the HST images. Our mass models are constructed based on GALFIT model fits. While this has the strength of properly incorporating a Tiny Tim synthetic image of the HST PSF, it also makes parametric assumptions about the NSC SB profiles (that they are welldescribed by Sérsic profiles). We test for systematic errors in this approach by directly fitting the HST images using the mge_fit_sectors IDL package (see footnote 11) after approximating the PSF as a circularly symmetric MGE as well. These MGEs result in the best fit of $\mathrm{BH}$ masses (and upper limits for NGC 205) that change by 7\%-15\%, with similar $\beta_{z}, \gamma$, and $i$. These best-fit results with multiple existing color $-M / L$ relations are presented in Table 12 , where the superscripts D and $\mathrm{G}$ stand for fitting their HST images and GALFIT models.

\subsubsection{Uncertainties Due to Kinematic PSF in the Spectroscopic Data of NGC 5206}

As discussed in Section 2.3, the PSF of our kinematic data of NGC 5206 has a larger uncertainty than our HST PSFs and the other kinematic PSFs due to a significant scattered-light component. The JAM for $M_{\mathrm{BH}}, \beta_{z}$, and $\gamma$ for NGC 5206 using the PSF derived from the half scattered-light subtraction gives $M_{\mathrm{BH}}=4.1_{-4.1}^{+2.6} \times 10^{5} M_{\odot}, \beta_{z}=0.30_{-0.35}^{+0.15}$, and $\gamma=1.09_{-0.09}^{+0.15}$ (assuming fixed $i=44^{\circ}$ ). So, along with the mass models, the PSF uncertainty also reduces the detection significance of this $\mathrm{BH}$.

\subsubsection{Dynamical Modeling Uncertainties}

We have used JAM modeling (Cappellari 2008) to dynamically model our galaxies. The agreement we find between our JAM modeling and previous measurements with the more flexible Schwarzschild modeling technique in M32 and NGC 205 (see above) is consistent with previous studies (e.g., Cappellari et al. 2009; Seth et al. 2014; Feldmeier-Krause et al. 2017; Leung et al. 2018), and JAMs have also been shown to give $\mathrm{BH}$ mass estimates consistent with maser estimates (Drehmer et al. 2015).

The two dynamical modeling techniques differ quite significantly. In both cases, deprojected light/mass models are used as inputs, but in the Schwarzschild models, these models are used to create a library of all possible orbits, and these orbits are then weighted to fit the mass model and full LOSVD (e.g., van den Bosch et al. 2008). This enables determination of the orbital structure as a function of radius (including anisotropy). In the JAMs, only the $V_{\text {rms }}$ is fit, and the Jeans anisotropic equations used make a number of simplifying assumptions; most notably, the anisotropy is fit as the parameter $\beta_{z}=1-\left(\sigma_{z}^{2} / \sigma_{R}^{2}\right)$ and is aligned with the cylindrical coordinate system (Cappellari 2008). We use constant 
Table 7

Dynamical NSC Properties

\begin{tabular}{|c|c|c|c|c|c|c|c|c|c|}
\hline $\begin{array}{l}\text { Object } \\
\text { (1) }\end{array}$ & $\begin{array}{l}\text { Filter } \\
\text { (2) }\end{array}$ & $\begin{array}{c}r_{\text {eff. }} \\
(\operatorname{arcsec}) \\
(3)\end{array}$ & $\begin{array}{l}r_{\text {eff. }} \\
(\mathrm{pc}) \\
(4)\end{array}$ & $\begin{array}{c}\mu_{\mathrm{NSC}} \\
(\mathrm{mag}) \\
(5)\end{array}$ & $\begin{array}{c}L_{\star} \\
\left(\times 10^{7} L_{\odot}\right) \\
(6)\end{array}$ & $\begin{array}{c}M_{\star, \text { dyn. }} \\
\left(\times 10^{7} M_{\odot}\right) \\
(7)\end{array}$ & $\begin{array}{c}M / L_{\text {pop., NSC }} \\
\left(M_{\odot} / L_{\odot}\right) \\
(8)\end{array}$ & $\begin{array}{c}\rho_{r_{\text {eff. }}} \\
\left(M_{\odot} \mathrm{pc}^{-3}\right) \\
(9)\end{array}$ & $\begin{array}{l}t_{\text {relaxation }} \\
\text { (yr) } \\
(10)\end{array}$ \\
\hline M32 & F814W & $1.1 \pm 0.1$ & $4.4 \pm 0.4$ & $11.0 \pm 0.1$ & $1.10 \pm 0.10$ & $1.65 \pm 0.31$ & 1.35 & $1.1 \times 10^{5}$ & $6.8 \times 10^{9}$ \\
\hline NGC 5102 & F547M & $1.6 \pm 0.1$ & $26.3 \pm 1.6$ & $12.1 \pm 0.2$ & $12.51 \pm 2.49$ & $7.30 \pm 2.34$ & 0.50 & $2.0 \times 10^{3}$ & $3.8 \times 10^{10}$ \\
\hline NGC 5206 & F814W & $0.5 \pm 0.1$ & $8.1 \pm 1.7$ & $14.4 \pm 0.2$ & $0.73 \pm 0.16$ & $1.54 \pm 0.51$ & 1.98 & $3.8 \times 10^{3}$ & $2.0 \times 10^{10}$ \\
\hline
\end{tabular}

Note. Column 1: host galaxy name. Column 2: filter used for the luminosity modeling. Columns 3 and 4: effective radii of NSCs in arcsec and pc. Column 5: total apparent magnitude of each NSC. Note that the total apparent magnitude of M32's and NGC 205's NSCs are taken from Table 5, while those of NGC 5102 and NGC 5206's NSCs are estimated from their two inner Sérsic components in Table 5. Columns 6 and 7: luminosities (in filter from column 2) and dynamical masses of NSCs. Here we assume the mass scaling factor $\gamma$ is calculated from the JAM dynamical models using the R15 color-M/L mass models. Column 8: color-based population $M / L$ in the band given in column 2 (see Table 5). Column 9: mass densities at the effective radii of the NSCs. Column 10: relaxation timescales of the nuclei evaluated at the effective radii of NSCs.

anisotropy models here. In practice, this assumption likely does not lead to significant inaccuracies, as the anisotropy has been found to be small and fairly constant in the MW (Schödel et al. 2009), M32 (Verolme et al. 2002), NGC 404 (Nguyen et al. 2017), and Cen A (Cappellari et al. 2009), as well as the more distant M60-UCD1 (Seth et al. 2014) and NGC 5102 and NGC 5206 in this work.

The lack of orbital freedom in the JAMs relative to the Schwarzschild models means that the error bars are typically smaller in the JAMs; Seth et al. (2014) found errors in BH mass from M60-UCD1 from JAMs that are 2-3 times smaller than the errors from the Schwarzschild models; for this reason, we quote conservative, $3 \sigma$ errors in this work.

\section{Nuclear Star Clusters}

\subsection{Dynamical NSC Masses}

We assume the NSCs are described by the innermost (M32 and NGC 205) or the inner + middle (NGC 5102 and NGC 5206) components of our GALFIT light profile models (see Table 5). To estimate the masses of the NSCs, we scale the population estimates for these components from our best-fit R15 MGEs using the best-fit dynamical models. The $1 \sigma$ errors in the $\gamma$ values are combined with the errors on the luminosities of the components to create the errors given in Table 7.

Our mass estimate for M32 is quite consistent with that of Graham \& Spitler (2009). This, however, appears to be a coincidence, as our NSC luminosity is $3 \times$ lower, while our dynamical $M / L$ is $3 \times$ higher than the stellar population value they assume. However, we note that the stellar synthesis estimates of Coelho et al. (2009) appear to match our colorbased estimates. In NGC 205, our mass estimate is somewhat higher than the dynamical estimate of $1.4 \times 10^{6} M_{\odot}$ in De Rijcke et al. (2006); their model assumed a constant $M / L$ with radius and a King profile for the NSC. Our dynamical mass of NGC 5102's NSC is an order of magnitude higher than the estimate of Pritchet (1979) in the $V$ band, in part because we use $M / L_{\mathrm{F} 547 \mathrm{M} \text {,dyn. }} \sim M / L_{V}=0.55\left(M_{\odot} / L_{\odot}\right)$ (Mitzkus et al. 2017) or $M / L_{\mathrm{F} 547 \mathrm{M} \text {,dyn. }}=M / L_{\mathrm{F} 547 \mathrm{M} \text {,pop. }} \times \gamma_{\mathrm{F} 547 \mathrm{M}}$ with $M / L_{\mathrm{F} 547 \mathrm{M} \text {, pop. }} \sim 0.5\left(M_{\odot} / L_{\odot}\right)$ estimated from the R15 color$M / L$ relation within 0 " 5 , while Pritchet (1979) adopted a very low $M / L_{V}=0.11\left(M_{\odot} / L_{\odot}\right)$.

To calculate the effective radii $\left(r_{\text {eff }}\right)$ of the multiplecomponent NSCs in NGC 5102 and NGC 5206, we integrate the two components together to get an effective radius of 0 !" 5 $(8 \mathrm{pc})$ for NGC 5206 and 1"!6 (26 pc) for NGC 5102. In

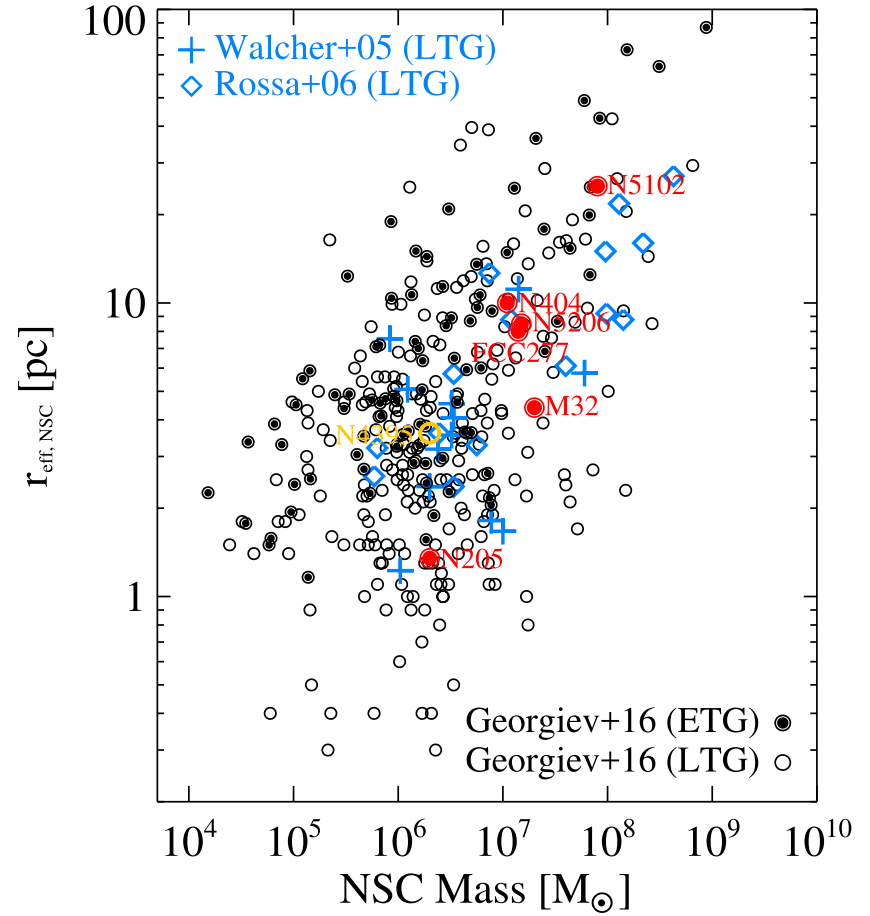

Figure 9. Mass vs. size for NSCs. Our dynamical mass determinations plus that of NGC 404 (Nguyen et al. 2017) are shown in red (circles with central dots); these are the only dynamically determined NSC masses in ETGs. These are compared to photometric estimates from Georgiev et al. (2016), plotted in black circles with central dots for ETGs and black open circles for LTGs. The LTG NSCs with the dynamical $M / L$ estimates from Walcher et al. (2005; latetype spirals) and the spectroscopic $M / L$ estimates from Rossa et al. (2006; Sa$\mathrm{Sbc}$ ) are plotted with blue plus signs and diamonds, respectively. We also add the dynamical $M / L$ estimate for the LTG NSC of NGC 4395 (den Brok et al. 2015) with a yellow open circle and the ETG NSC of FCC 277 (Lyubenova et al. 2013) in the same notation of this work's NSCs.

Figure 9, we compare the sizes and dynamical masses of our ETG NSCs to previous measurements in other galaxies. Most of the previous measurements are photometric mass estimates taken from Georgiev et al. (2016). The dynamical $M / L$ estimates are taken from Walcher et al. (2005) for LTGs, Lyubenova et al. (2013) for FCC 277, den Brok et al. (2015) for NGC 4395, and Nguyen et al. (2017) for NGC 404, and the spectroscopic $M / L$ estimates are taken from Rossa et al. (2006) for LTGs; we note here that our data are the first sample of dynamical NSC masses measured in ETGs. We find that all four of our NSCs fall within the existing distribution in the 
size-mass plane, with M32 and NGC 205 being on the compact side of the distribution.

\subsection{Uncertainties on NSC Mass Estimates}

For the quoted errors in the NSC masses shown in Table 7, we take the $3 \sigma$ errors on the mass scaling factors and combine these in quadrature with the uncertainties in the total luminosity of the NSCs to provide a conservative error estimate. The uncertainty in the total luminosity of the NSC (which comes from how much of the center is considered to be part of the NSC) ends up being the dominant error. The total fractional errors range between $11 \%$ and $21 \%$. In addition to these errors, we consider the systematic error on the mass estimates from using different color $-M / L$ relations. We find that the maximum differences are $<12 \%$; given that these are below the quoted uncertainties in all cases, we do not quote separate systematic errors.

\subsection{Structural Complexity and NSC Formation}

The NSCs of NGC 5102 and NGC 5206 have complex morphologies made up of multiple components, while NGC 205 also shows clear color gradients within the nucleus. A similarly complex structure has also been seen in the NSC of nearby ETG NGC 404, where a central light excess in the central $0.22(3 \mathrm{pc})$ appears to be counterrotating relative to the rest of the NSC (Seth et al. 2010) and has an age of $\sim 1 \mathrm{Gyr}$ (Nguyen et al. 2017). The sizes of NSCs in spirals also change with wavelength, with bluer bands having smaller sizes and being more flattened, suggesting that young populations are concentrated near the center (Seth et al. 2006; Georgiev \& Böker 2014; Carson et al. 2015). In NGC 5102, the central component dominates the light within the central 0 "! 2 (3 pc), while in NGC 5206, the central component dominates the light out to 0 ". $5(8 \mathrm{pc})$. However, it is challenging to determine if these are in fact distinct components. In both NSCs, the color gets bluer near the center, suggesting a morphology similar to that seen in the late-type NSCs. In NGC 5102, the outer component is somewhat more flattened and has an elevated $V / \sigma$ value, suggesting that it may be more strongly rotating than the inner component. In NGC 5206, both components have similar flattening and PAs, and no clear rotation kinematic differences are seen.

As NSCs have complex stellar populations, it is not surprising that their morphology may also be complex. These multiple components are likely linked to their formation history. In particular, the central bluer/younger components seen in $3 / 4$ of our clusters strongly argue for in situ star formation, as inspiraling clusters are expected to be tidally disrupted in the outskirts of any preexisting cluster (e.g., Antonini 2013). The gas required for in situ star formation could be funneled into the nucleus by dynamical resonances in the galaxy disk (e.g., Schinnerer et al. 2003) or produced within the NSC by stellar winds (Bailey 1980) or collisions (Leigh et al. 2016). The large $r_{\text {eff }}=32 \mathrm{pc}$, high-mass fraction ( $1 \%$ of total galaxy mass) outer component in NGC 5102 may also be akin to the extra-light components expected to be formed from gas inflows during mergers (Hopkins et al. 2009). Regardless of the source of the gas, it is clear that NSCs in ETGs frequently host younger stellar populations within their centers, similar to late-type NSCs.

\section{Discussion}

\subsection{BH Demographics}

We have dynamically constrained the BH masses of M32, NGC 205, NGC 5102, and NGC 5206. While we find no evidence of a BH in NGC 205 (our $3 \sigma$ upper limit is $7 \times 10^{4} M_{\odot}$ ), we detect BHs in the other three systems. Two of these best-fit BHs are below $10^{6} M_{\odot}$, in NGC 5206 $\left(4.7 \times 10^{5} M_{\odot}\right)$ and NGC $5102\left(8.8 \times 10^{5} M_{\odot}\right)$. This doubles the previous sample of $\mathrm{BHs}$ with dynamical constraints placing them below $10^{6} M_{\odot}$; the previous two were found in late-type spiral NGC 4395 (den Brok et al. 2015) and the accreting BH in NGC 404 (Nguyen et al. 2017). This adds significantly to the evidence that $\lesssim 10^{6} M_{\odot}$ BHs do inhabit the nuclei of lowmass galaxies (see also Reines \& Volonteri 2015).

Combining our results with the accretion evidence for a $\mathrm{BH}$ in NGC 404 (Nguyen et al. 2017), we can make a measurement of the fraction of the nearest ETGs that host BHs. These five galaxies have total stellar masses between $5 \times 10^{8}$ and $10 \times 10^{9} M_{\odot}$. This sample represents a complete, volumelimited sample of ETGs in this mass range and thus forms a small but unbiased sample of ETGs (Section 3). Most of the galaxies are satellite galaxies, with only NGC 404 being isolated; thus, these represent only group and isolated environments. With four out of five of these galaxies now having strong evidence for central $\mathrm{BHs}$, we measure an occupation fraction of $80 \%$ over this stellar-mass range.

We can directly compare this to the occupation fraction estimates from the AMUSE survey of X-ray nuclei in ETGs (Gallo et al. 2008; Miller et al. 2015). Of the 57 galaxies observed in the mass range of our ETGs, only nine (16\%) have detected X-ray sources; however, modeling of the X-ray luminosity function suggests an occupation fraction of 30\%$100 \%$ for these galaxies (Miller et al. 2015). Thus, both dynamical and accretion evidence points to a high-mass occupation fraction for ETGs down to $\sim 10^{9} M_{\odot}$.

The high occupation fraction we find here can be used to constrain the seed formation mechanism of BHs in the early universe. Our result appears to be more consistent with the scenario where BH seeds are formed from the remnants of the first generation of massive stars (e.g., Population III stars (light seeds); Volonteri et al. 2008; Volonteri 2010; Greene 2012; Reines \& Comastri 2016), rather than heavier seeds. More specifically, the $80 \%$ observed occupation fraction is consistent with the semi-analytic model predictions at $z=0$ for light seeds as a function of velocity dispersion (Volonteri et al. 2008; van Wassenhove et al. 2010). A more recent semi-analytic prediction predicts a significantly lower $\mathrm{BH}$ occupation of $\sim 20 \%$ in our mass range for the low-mass seed scenario (Antonini et al. 2015a). This study also breaks down the prediction by galaxy type, with an even lower occupation fraction expected in similar-mass LTGs. We note that our measurements apply only to ETGs, which make up a minority of galaxies at the stellar masses probed (e.g., Blanton et al. 2005).

Our BH masses are also consistent with their formation from runaway tidal capture of NSC stars, a scenario outlined by Stone et al. (2017). Stone et al. (2017) found that this runaway tidal capture process requires dense NSCs that are typically found only in galaxies with $\sigma \gtrsim 35 \mathrm{~km} \mathrm{~s}^{-1}$. For sufficiently dense NSCs (in higher-dispersion galaxies), the runaway tidal encounter supplies mass for the BH seed to grow, 


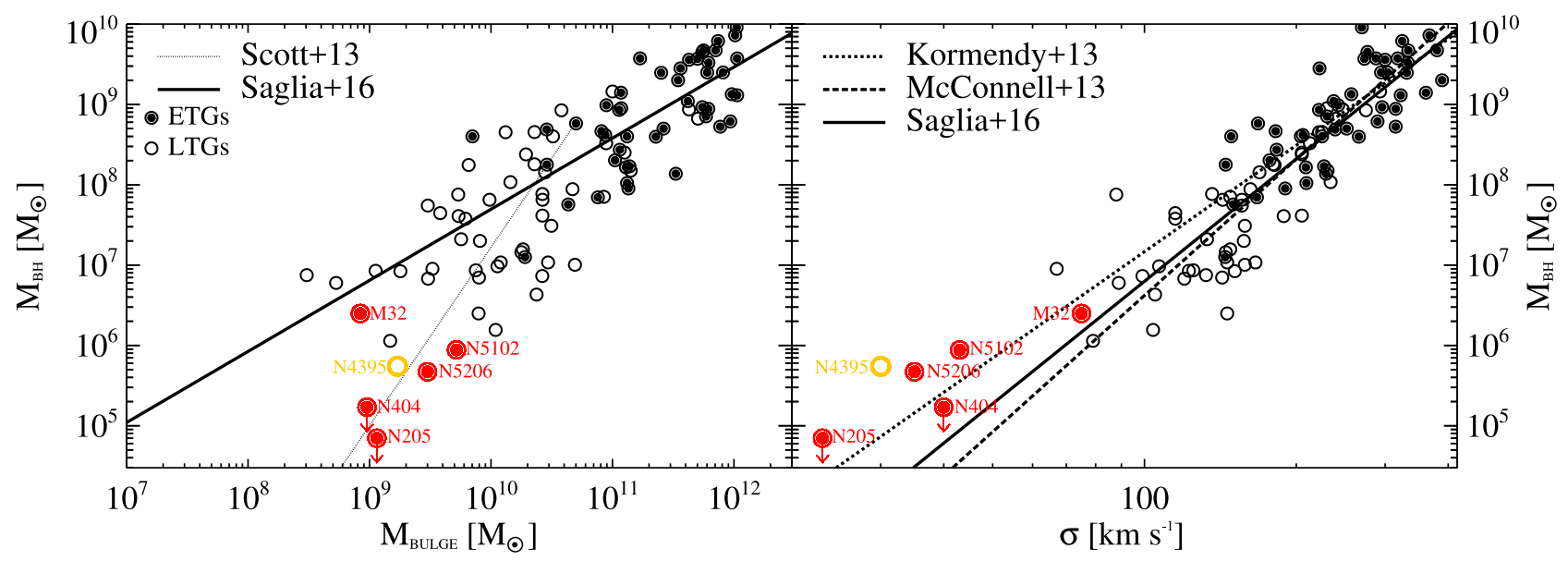

Figure 10. Our four low-mass ETGs in the context of the $M_{\mathrm{BH}}-M_{\text {Bulge }}$ (left) and $M_{\mathrm{BH}}-\sigma$ (right) scaling relations. The data of both ETGs (black dots within open circles) and LTGs (black open circles) are taken from the compilation of Saglia et al. (2016). The dotted, dashed, long-dashed, and solid lines indicate their linear best fit in log scale of the relations from Scott et al. (2013), Kormendy \& Ho (2013), McConnell \& Ma (2013), and Saglia et al. (2016) for ETGs (LTGs), respectively. The BH masses of M32, NGC 5102, and NGC 5206 and $3 \sigma$ upper limit mass of NGC 205 with the downward arrow are plotted with red dots within open circles, as in the legend. We also add BHs with dynamical masses below $10^{6} M_{\odot}$, including the dwarf AGN LTG NGC 4395 (yellow open circle; den Brok et al. 2015) and ETG NGC 404 (Nguyen et al. 2017).

and this growth is larger for higher-dispersion galaxies. Over a Hubble time, this BH growth saturates at a specific mass. Using their Equation (37), we find saturation masses of $\sim 6.5 \times 10^{5}$, $\sim 5 \times 10^{5}$, and $\sim 1 \times 10^{6} M_{\odot}$ for NGC 5102, NGC 5206, and M32, respectively, using the velocity dispersions estimated in Section 8.2. These BH masses are within a factor of $\sim 2$ of the BH masses we dynamically measure in Section 6.1. NGC 205 and NGC 404 both fall below the threshold dispersion where runaway tidal growth is expected, and our upper mass limits in these galaxies are therefore also consistent with this scenario.

Additional constraints on the occupation fraction in a wider range of galaxy types are possible in the local volume, and in future work, we plan to provide additional constraints using existing data on LTGs with both stellar kinematics using Gemini/NIFS or VLT/SINFONI and upcoming gas kinematic data from ALMA.

\subsection{BH Mass Scaling Relations}

We examine our best-fit BH masses in the context of scaling relations including $M_{\mathrm{BH}}-M_{\text {Bulge }}$ and $M_{\mathrm{BH}}-\sigma$ (Kormendy \& Ho 2013; McConnell \& Ma 2013; Saglia et al. 2016) for ETGs. Since our dynamical constraints suggest $\gamma \sim 1$, and we do not have direct dynamical measurements of the bulge masses, we assume population-based masses for the bulge components here for all four galaxies; these are listed in Column 12 of Table 5.

We note that we assume that the galactic bulges are comprised of all two (NGC 205 and M32) and three (NGC 5102 and NGC 5206) Sérsic components. Kormendy \& Ho (2013), McConnell \& Ma (2013), and Saglia et al. (2016) reported that the bulge mass and luminosity of M32 are $\sim 4.5 \times 10^{8} M_{\odot}$ and $\sim 4 \times 10^{8} L_{\odot}\left(M_{V}=-16.64\right)$, while our estimates of mass and luminosity from $\mathrm{F} 814 \mathrm{~W}$ are $M_{\text {Bulge }}$ $\sim 8.2 \times 10^{8} M_{\odot}$ and $L_{\text {Bulge }} \sim 4.5 \times 10^{8} L_{\odot}$; these are consistent with Graham \& Spitler (2009) and Kormendy \& Bender (2009) estimated in the $I$ band. For NGC 205, McConnachie (2012) reported a total luminosity of $M_{V}=-16.5$ (3 $\times$ $10^{8} L_{\odot}$ ), and the Angus et al. (2016) simulation gives a mass of $4.0 \times 10^{8} M_{\odot}$. We find a consistent luminosity but higher mass for the bulge of $6.5 \times 10^{8} M_{\odot}$ from F814W HST data.
Figure 10 shows that the best-fit $\mathrm{BH}$ mass of M32 is within the scatter of the $M_{\mathrm{BH}}-M_{\mathrm{Bulge}}$ relations of Kormendy \& Ho (2013), McConnell \& Ma (2013), and Saglia et al. (2016). However, the best-fit BHs masses of NGC 205, NGC 5102, and NGC 5206 clearly fall below these $M_{\mathrm{BH}}-M_{\text {Bulge }}$ relations by 1-2 orders of magnitude. Combined with other recent work on BH mass measurements in low-mass systems, e.g., NGC 404 (Nguyen et al. 2017), NGC 4395 (den Brok et al. 2015), RGG 118 (Baldassare et al. 2015), and Pox 52 (Barth et al. 2004; Thornton et al. 2008), an increasing number of galaxies appear to fall below the BH mass-versus-bulge mass relationship extrapolated from massive ETGs to the low-mass regime. This suggests that the $M_{\mathrm{BH}}-M_{\mathrm{Bulge}}$ relationship may steepen as suggested by Scott et al. (2013) for low-mass systems with bulge mass $<2 \times 10^{10} M_{\odot}$. Alternatively, the scaling relations may just break down at these masses, with significant scatter to lower masses, as also seen in the megamaser BH measurements in spiral galaxies (Greene et al. 2016; Läsker et al. 2016a). We note that this increased scatter may occur at bulge masses where the occupation fraction also drops below unity.

Next, we explore these BHs in the context of the $M_{\mathrm{BH}}-\sigma$ scaling correlation. While typically, the dispersion used is that estimated within the effective radius, we do not have this information for all of our galaxies. For M32 and NGC 205, the $\sigma_{e}$ were measured by Ho et al. (2009), who measured the dispersions of $72.1 \pm 1.9$ and $23.3 \pm 3.7 \mathrm{~km} \mathrm{~s}^{-1}$ in a $2^{\prime \prime} \times 4^{\prime \prime}$ aperture, respectively. To estimate the central velocity dispersions of NGC 5102 and NGC 5206, we coadd all the spectra in the FOV of 1.15 and fit the resulting single spectrum with pPXF for velocity dispersions. This gives dispersions of $42.3 \pm 1.1 \mathrm{~km} \mathrm{~s}^{-1}$ for NGC 5102 and $35.4 \pm 1.0 \mathrm{~km} \mathrm{~s}^{-1}$ for NGC 5206.

\subsection{Possibility of Collections of Dark Remnants}

Recent work has suggested that mass segregation of stellarmass BHs can occur on timescales well below the relaxation time (Bianchini et al. 2017), significantly enhancing the central $M / L$ of globular clusters. An enhancement of $M / L$ is in fact seen in the globular cluster M15 (den Brok et al. 2014b). This 


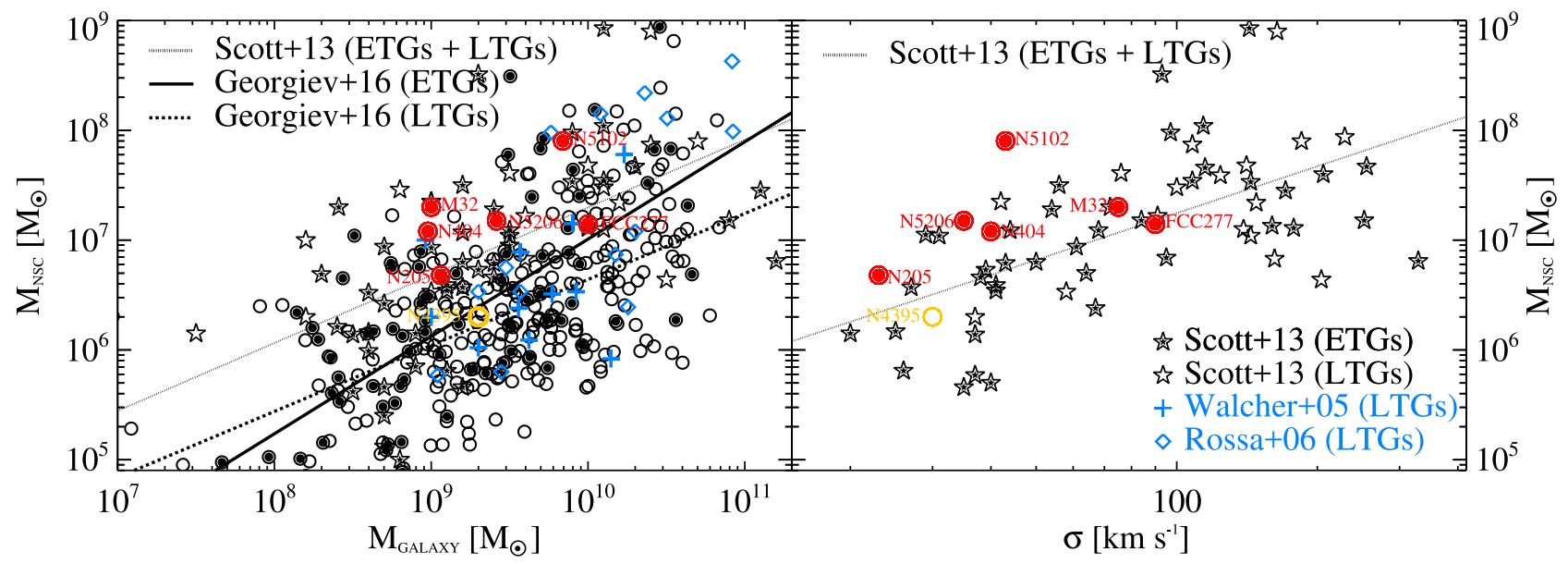

Figure 11. Our four galaxies in the context of the $M_{\mathrm{NSC}}-$ galaxy mass (left) and $M_{\mathrm{NSC}}-\sigma$ (right) scaling relations. The data are taken from ETGs (double stars) and LTGs (open stars; Scott et al. 2013; Georgiev et al. 2016). ETGs are plotted with black dots within circles, while LTGs are plotted with open circles; and their corresponding linear best-fit Mnsc-Mgalaxy scaling relation in log scale. We note that the Scott et al. (2013) linear relation has been fit to the sample of both ETGs (solid line) and LTGs (dashed line). The M32, NGC 205, NGC 5102, and NGC 5206 NSCs are shown with colored dots with circles, as in the legend. We also add two more NSCs: LTG NGC 4395 (den Brok et al. 2015) and ETG NGC 404 (Nguyen et al. 2017). The NSCs have dynamical or spectroscopic M/L estimates from Walcher et al. (2005; late-type spirals) and Rossa et al. (2006; Sa-Sbc) that are plotted with gray plus signs and diamonds, respectively. We also add the dynamical estimate of the ETG NSC of FCC 277 (Lyubenova et al. 2013) in the same notation of this work's NSCs. The total stellar mass of FCC 277 was estimated assuming its $B$-band absolute magnitude of -17.78 (http://leda.univ-lyon1.fr/search.html) and $M / L_{B} \sim 5.0\left(M_{\odot} / L_{\odot}\right)$ at the highest $3 \sigma$ of its Schwarzschild dynamical estimate in the HST/ACS $z$ band (Lyubenova et al. 2013).
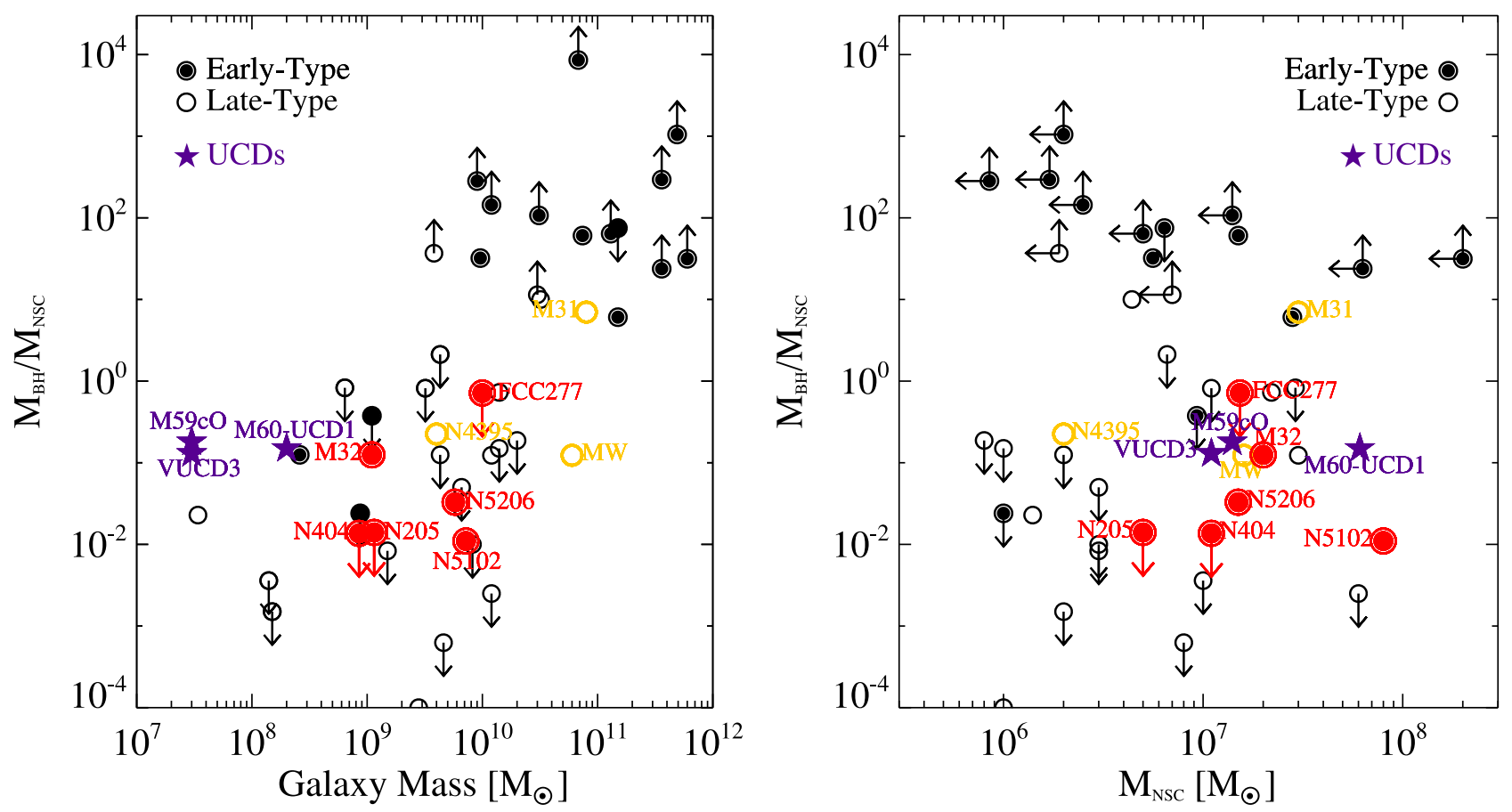

Figure 12. Ratio of the BH and NSC mass against their host galaxy stellar mass (left) and NSC dynamical masses based on dynamical measurements of $M / L_{\text {dyn. }}$ (right). Original data with dynamical measurements are shown with ETGs (black dots within circles) and LTGs (black open circles), and current BH mass measurements of UCDs (purple stars) are taken from Ahn et al. (VUCD3 and M59cO; 2017), Graham \& Spitler (2009), Lyubenova et al. (FCC 277; 2013), Neumayer \& Walcher (2012) and Seth et al. (M60-UCD1; 2014). The upward/downward arrows are used for galaxies with lower/upper limits of BH and NSC masses. We note that for the UCD NSC masses, we plot only the masses of their inner components.

leads to the possibility that a significant number of stellar-mass $\mathrm{BHs}$ could collect at the center of NSCs, leading to a false detection of a massive BH. We note that the current simulations are of much lower-mass systems than the massive NSCs we discuss here, and that significant uncertainties exist on how many BHs would segregate and be retained during this process. Nonetheless, we can get a sense of the maximum dark mass that can plausibly be accumulated by stellar remnants. For a solar-metallicity population with a standard Kroupa IMF, $\sim 4 \%$ of the mass of the cluster will turn into stellar-mass BHs (Shanahan \& Gieles 2015). This represents a maximum mass fraction of the NSCs that could be concentrated at the center, with the retention fraction of BHs likely being much lower than this. The fraction of the NSC mass found in the BHs in M32, 

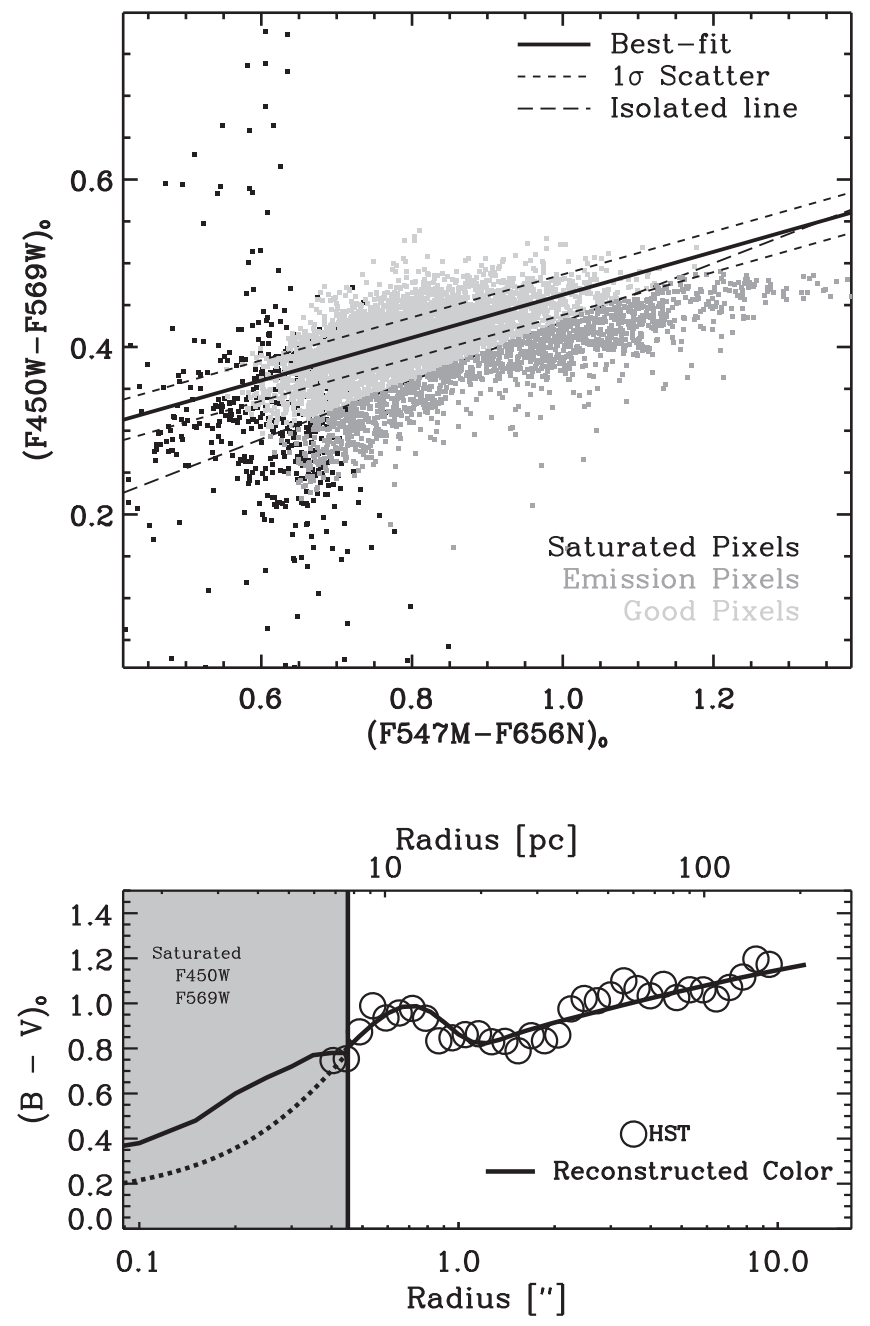

Figure 13. Top panel: F450W-F569W vs. F547M-F656N color-color correlation for the NGC 5102 nucleus. Black dots are the saturated pixels within $0 . / 4$ in F450W and F569W. Gray dots are the pixels that are possibly contaminated by $\mathrm{H} \alpha$ emission in $\mathrm{F} 656 \mathrm{~N}$ band. Light gray dots are pixels outside 0 ". 4 , which are used for the fit. The solid line is the best fit of the $(\mathrm{F} 450 \mathrm{~W}-\mathrm{F} 569 \mathrm{~W})_{0}$ vs. (F547M-F656N) $)_{0}$ relation, while the dashed lines represent the $1 \sigma$ scatter of the best fit. The long-dashed line shows the isolation criterion of $\mathrm{H} \alpha,(\mathrm{F} 547 \mathrm{M}-\mathrm{F} 656 \mathrm{~N})_{0} \gtrsim 2.5 \times(\mathrm{F} 450 \mathrm{~W}-\mathrm{F} 569 \mathrm{~W})_{0}$. Bottom panel: the $(B-V)_{0}$ color profile, which is inferred from the HST photometric transformation from WFPC2 $\rightarrow B V R I$ (Sirianni et al. 2005). Circles are the $H S T$ data outside $0 . " 4$, and the gray shaded region is the saturated HST data within 0 ." 4 . The solid line is the best-fit model of the $(B-V)_{0}$ colors, while the dashed line is the original saturated color.

NGC 205, NGC 5102, and NGC 5206 is $13 \%,<3.5 \%, 1.1 \%$, and $3.3 \%$ of their NSC masses, respectively. Therefore, based on the mass fractions, apart from M32, a collection of dark remnants is a possibility.

We can also evaluate stellar relaxation times $\left(t_{\text {relaxation }}\right)$ of these nuclei at the effective radii of their NSCs using the following equation (Bahcall \& Wolf 1977; Valluri et al. 2005):

$$
t_{\text {relaxation }} \simeq\left(1.4 \times 10^{8}\right) \sigma_{20}^{3} \rho_{5}^{-1}\left(\ln \Lambda_{10}\right)^{-1}(\text { years }),
$$

where $\sigma_{20}=\sigma_{\star} / 20 \mathrm{~km} \mathrm{~s}^{-1}, \rho_{5}=\left(\rho_{\mathrm{g}, \mathrm{dyn} .}\right) / 10^{5} M_{\odot} \mathrm{pc}^{-3}, \ln \Lambda_{10}=$ $\ln \Lambda / 10$, and $\ln \Lambda=10$. Here $\sigma_{\star}$ is the stellar dispersion estimated in Section 8.2, and the mass densities $\rho_{\mathrm{g}, \text { dyn }}$ are estimated at the effective radii of NSCs. These mass densities and relaxation times are provided in Table 7. Our estimated relaxation times for the $\mathrm{BH}$ detections are long (6.8-38 Gyr), while in NGC 205 (the one source without a $\mathrm{BH}$ detection), the relaxation time is quite short $(0.58 \mathrm{Gyr})$. The fact that we detect no $\mathrm{BH}$ in this shortestrelaxation-time system (as well as the tight upper limit on the mass of the M33 BH; Gebhardt et al. 2001) argues against the detected BHs being collections of remnants. However, more realistic simulations are required to evaluate whether significant fractions of the NSC BHs can really be mass-segregated and retained.

\subsection{NSC Masses and Scaling Relations}

Our dynamical mass measurements of the four NSCs in this work, along with measurements of the NGC 404 NSC (Seth et al. 2010; Nguyen et al. 2017) and FCC 277 (Lyubenova et al. 2013), are the first six dynamical mass measurements of NSCs in ETGs. These NSC masses are plotted against the mass and dispersion of galaxies in Figure 11.

Comparing our dynamical NSC masses to the galaxy stellar masses and dispersions, we find that they are within the scatter of the previous (mostly photometric) NSC mass measurements. However, almost all of the dynamical measurements lie on or above previous relations, with NGC 5102 being the highestmass outlier. The closest previously published relations are those presented in Scott et al. (2013). The Georgiev et al. (2016) NSC-galaxy mass relation for ETGs lies below all six dynamical measurements.

The ratios between the NSCs masses and their host bulge/ galaxy masses are $\sim 1.7 \%, 0.2 \%, 1.1 \%, 0.7 \%$, and $<1.5 \%$ for M32, NGC 205, NGC 5102, NGC 5206, and NGC 404 (Nguyen et al. 2017), respectively. Apart from NGC 205, these NSCs are more massive than the typical $\sim 0.3 \%$ mass fraction for NSCs seen in ETGs with similar stellar masses in the Virgo cluster (Côté et al. 2006; Spengler et al. 2017). A trend toward higher-mass NSCs in lower-mass galaxies was suggested by Graham \& Spitler (2009) and is verified for ETGs in recent work (Spengler et al. 2017); based on the Graham \& Spitler (2009) relation, a mass fraction of $\sim 1 \%$ is expected for our sample galaxies. The high masses and mass fractions inferred here might be a sign that the relation of NSC to galaxy mass changes outside cluster environments, as might be expected based on the increased availability of gas to accrete to the present day.

\subsection{Relative Formation between NSCs and BHs}

As discussed in the Introduction, the relationship between NSCs and BHs is not yet clear. Our galaxies add significantly to the sample of galaxies where we have mass measurements for both the $\mathrm{BH}$ and NSC. Our three $\mathrm{BH}$ detections have an $M_{\mathrm{BH}} / M_{\mathrm{NSC}}$ ratio between $10^{-2}$ and 0.3 . We plot these against the galaxy and NSC masses in Figure 12 . The $M_{\mathrm{BH}} / M_{\mathrm{NSC}}$ ratio in our galaxies is consistent with galaxies like the MW (e.g., Schödel et al. 2014) and previous measurements of UCDs (Seth et al. 2014; Ahn et al. 2017), where just the inner component of the UCD is assumed to be the remnant NSC. However, the lack of NSCs in massive ETGs clearly separates these from our sample (Neumayer \& Walcher 2012). On the other hand, for spiral galaxies with upper limits on the BHs (Neumayer \& Walcher 2012), our NGC 205 limit joins previous measurements constraining the $\mathrm{BH}$ mass to be at most $10^{-2}-10^{-3}$ that of the NSC. Even amongst the sample where both BHs and NSCs are found, there is a scatter of $\sim 3$ orders of magnitude at 
a similar NSC mass. This wide range of $M_{\mathrm{BH}} / M_{\mathrm{NSC}}$ ratios suggests that NSC and $\mathrm{BH}$ formation must not always be tightly linked.

\subsection{Relation of NSCs to UCDs}

The infall and ongoing stripping of the Sagittarius dwarf spheroidal galaxy shows that nuclei similar to those we sample here can be stripped of their host galaxies (e.g., Monaco et al. 2005). Our NSCs clearly overlap with the radii and mass distribution of UCDs (Norris et al. 2014), and some of these UCDs may be stripped galaxy nuclei (e.g., Pfeffer et al. 2014). The UCDs typically have higher-than-expected integrated dispersion (e.g., Haşegan et al. 2005). Specifically, virial mass estimates based on these dispersions give $M / L$ s that are higher than expected from their stellar populations-these can be interpreted as indirect evidence for massive BHs (Mieske et al. 2013), as would be expected if UCDs were tidally stripped NSCs. The presence of these BHs with mass fractions above $10 \%$ has been verified in a few massive UCDs $\left(>10^{7} M_{\odot}\right)$ using resolved kinematic measurements (Seth et al. 2014; Ahn et al. 2017). We can examine the apparent increase in $M / L$ of our galaxies if we were to ignore the BHs by looking at the no-BH model $M / L$ s. These are inflated by $4 \%-10.0 \%$ in our three galaxies with detected BHs; this is smaller than the effect observed in known UCDs due to the smaller mass fractions of the BHs $(1 \%-13 \%)$ in these systems. We note that the UCDs are often observed to have multiple components; thus, the NSCs may make up only a fraction their total mass, suggesting even lower $\mathrm{BH}$ mass fractions if these galaxies were to be stripped.

\section{Conclusions and Future Prospects}

\subsection{Conclusions}

We have examined the nuclear morphology and kinematics of a sample of four ETGs with stellar masses of $10^{9}-10^{10} M_{\odot}$ using adaptive optics Gemini/NIFS and VLT/SINFONI spectroscopic data and HST imaging. We use HST data to estimate the sizes, masses, and luminosities of their NSCs. Using dynamical modeling of the spectroscopic data, we constrain the masses of their central BHs and NSCs. Our primary findings are as follows.

1. Our dynamical models yield significant detection of central BHs in M32, NGC 5102, and NGC 5206 and an upper limit in NGC 205. The NGC 205 upper limit and $\mathrm{M} 32 \mathrm{BH}$ masses are consistent with previous measurements.

2. We present the first dynamical measurements of the central BH masses in NGC 5102 and NGC 5206. Both BHs have masses below 1 million $M_{\odot}$, thus doubling the number of dynamical measurements of sub-million $M_{\odot}$ BHs (den Brok et al. 2015; Nguyen et al. 2017).

3. Including our recent work on the BH in NGC 404 (Nguyen et al. 2017), we find that the fraction of $10^{9}-10^{10}$ $M_{\odot}\left(\sigma_{\star}=20-70 \mathrm{~km} \mathrm{~s}^{-1}\right)$ ETGs that host central BHs is at least $80 \%$ (4/5 with detected $\mathrm{BHs}$ ). These galaxies represent a volume-limited sample of ETGs in this mass range. This rather high occupation fraction suggests that the early universe produced an abundance of BH seeds, favoring a scenario where $\mathrm{BH}$ seeds formed from Population III stars (e.g., Volonteri 2010). Our BH masses are also consistent with growth by runaway tidal encounters (Stone et al. 2017).

4. The effective radii of the NSCs are $\sim 2-26 \mathrm{pc}$. The NSC SB profiles of NGC 205 and M32 are both fit well by a single Sérsic function, but the NSCs of NGC 5102 and NGC 5206 appear to have more complicated nuclear structures requiring two Sérsic components.

5. The high-quality ( $\sim 0$ " 1 resolution) kinematics of M32 and NGC 5102 show strong rotation $(V / \sigma \gtrsim 0.7)$, while NGC 205 and NGC 5206 have less significant rotation $(V / \sigma \sim 0.3)$.

6. We measure the masses of the NSCs in all four galaxies dynamically, some of the first dynamical mass estimates of ETG NSCs. Their dynamical masses range from $0.2 \times 10^{7}$ to $7.3 \times 10^{7} M_{\odot}$. These NSC masses are higher than the typical ETGs of similar galaxy mass in the Virgo and Fornax clusters, suggesting a possible environmental dependence on the NSC mass.

7. The BHs and NSCs in these galaxies appear to follow the $M_{\mathrm{BH}}-\sigma$ and $M_{\mathrm{NSC}}-\sigma$ relations. Their BHs fall below the bulge mass/total stellar mass-BH mass relations, while their NSCs are located above similar relations.

\subsection{Future Prospects}

Our dynamical modeling using high-resolution stellar kinematics from NIFS and SINFONI spectroscopy confirms the existence of a $\mathrm{BH}$ in $\mathrm{M} 32$ and puts an upper limit on NGC 205's BH mass. We also measure the masses of BHs in NGC 5102 and NGC 5206 for the first time. However, we model these $\mathrm{BH}$ masses assuming a constant $M / L$ or the $\mathrm{B} 03$ and $\mathrm{R} 15$ color $-M / L$ relations. Although the assumption of a constant $M / L$ is good for M32, the nuclei of NGC 205, NGC 5102, and NGC 5206 show clear spatial population variations and hence their nuclear $M / L s$. We will model these $M / L$ variations using new STIS data obtained this year using the techniques we developed in Nguyen et al. (2017). These data will enable stellar population synthesis modeling on the same 0 ".05 scale as our kinematic observations. With this information, we can significantly reduce the uncertainties in the stellar-mass profiles and the resulting $\mathrm{BH}$ mass estimates. Moreover, our upcoming ALMA data will also allow us to measure the central $\mathrm{BH}$ masses using cold gas kinematic models of the circumnuclear gas disk within the nuclei of a variety of Hubble-type, nearby, and low-mass galaxies. This larger and high-quality data sample will provide a more precise, complete, and unbiased occupation fraction dynamically.

The authors are grateful to Professor Elena Gallo of the University of Michigan, Department of Astronomy, for her refereeing role and provided comments that significantly improved the presentation and accuracy of the paper. We also would like to thank Aaron Barth of the Department of Physics and Astronomy, University of California- Irvine, and Luis Ho and Li Zhao-Yu of the Department of Astronomy, School of Physics, Peking University, for generously sharing with us their NGC 5102 and NGC 5206 CGS SB profiles in four Bessel-Johnson filters $B V R I$; Iskren Y. Georgiev of the Max-Planck Institut für Astronomie for providing his NSC data; and the Physics and Astronomy Department, 
University of Utah, for supporting this work. DDN and ACS acknowledge financial support from NSF grant AST1350389. MC acknowledges support from a Royal Society University Research Fellowship.

Facilities: Gemini: Gemini/NIFS/ALTAIR, ESO-VLT/ SINFONI, and HST(WFPC2, ACS HRC).

\section{Appendix A \\ Measurement of the NGC 5102 Nucleus $(B-V)_{0}$ Color Profile}

We determine the $(B-V)_{0}$ colors of NGC 5102 inside the radii of 0 ". 4 from $\mathrm{F} 450 \mathrm{~W}-\mathrm{F} 569 \mathrm{~W}$ WFPC2 data. However, these data are saturated at the center. We can use the F547MF656N data to predict the central $(B-V)_{0}$ colors where there does not appear to be strong $\mathrm{H} \alpha$ emission. The top panel of Figure 13 shows the scheme that we use to explore the $\lesssim 0$ ". 4 $(B-V)_{0}$ colors. First, we create the $(\mathrm{F} 450 \mathrm{~W}-\mathrm{F} 569 \mathrm{~W})_{0}$ and $(\mathrm{F} 547 \mathrm{M}-\mathrm{F} 656 \mathrm{~N})_{0}$ color maps with PSF cross-convolution and Galactic extinction correction in each band. Second, we get rid of all the colors with saturated pixels from F450W and F569W in both (F450W-F569W) $)_{0}$ and (F547M-F656N) $)_{0}$ maps (black dots). The pixels with possible contamination by $\mathrm{H} \alpha$ emission in the $\mathrm{F} 656 \mathrm{~N}$ band were also eliminated based on the criterion of $(\mathrm{F} 547 \mathrm{M}-\mathrm{F} 656 \mathrm{~N})_{0} \gtrsim 2.5 \times(\mathrm{F} 450 \mathrm{~W}-\mathrm{F} 569 \mathrm{~W})_{0}$ (gray dots under the long-dashed line). All of the good pixels are presented with light gray dots. Third, we use these good pixels of colors to fit the linear correlation between $(\mathrm{F} 450 \mathrm{~W}-\mathrm{F} 569 \mathrm{~W})_{0}$ and $(\mathrm{F} 547 \mathrm{M}-\mathrm{F} 656 \mathrm{~N})_{0}$. The best fit is presented by the solid black line, while the scatter by $1 \sigma$ of the fit is shown by the dashed black lines. The central $(\mathrm{F} 450 \mathrm{~W}-\mathrm{F} 569 \mathrm{~W})_{0}$ color profile is inferred from this $(\mathrm{F} 450 \mathrm{~W}-\mathrm{F} 569 \mathrm{~W})_{0}-(\mathrm{F} 547 \mathrm{M}-\mathrm{F} 656 \mathrm{~N})_{0}$ correlation.

We transform the $(\mathrm{F} 450 \mathrm{~W}-\mathrm{F} 569 \mathrm{~W})_{0}$ colors into the $(B-V)_{0}$ colors using the HST photometric transformation from WFPC2 $\rightarrow B V R I$ (Sirianni et al. 2005). The $(B-V)_{0}$ colors include the central region $(\lesssim 0$ !" 4$)$ that is shown in the bottom panel of Figure 13 . We plot the $(B-V)_{0}$ color profile of the outer part with radii $>0$ ". 4 from the $B$ (transformed from HST F450W) and $V$ bands (transformed from HST F569W; circles for data and solid line for the best-fit 1D model), while the saturated $(B-V)_{0}$ within the radii $r \lesssim 0$ " 4 is shown by the gray region and the $1 \mathrm{D}$ profile is shown by the dotted line. The best-fit reconstruction of $(B-V)_{0}$ is presented by the solid line. This new color profile results in a smaller mass of the NGC $5102 \mathrm{NSC}$ of $M_{\mathrm{NSC}}=(8.0 \pm 1.3) \times 10^{7} M_{\odot}, \sim 23 \%$ smaller than its mass with the assumption of constant $(V-I)_{0} \sim 1$ in the $\lesssim 1^{\prime \prime}$ region inferred from the groundbased observation (CGS) but totally consistent with the dynamical estimate in Table 7. Also, we note that we use this
$(B-V)_{0}$ color profile to model the mass model for NGC 5102 throughout this paper.

\section{Appendix B}

Kinematics Tables, MGE Models, and JAM Results

We make the full kinematic data set of NGC 205, NGC 5102, and NGC 5206 available in Tables 8-10, respectively, for use in future studies. Table 11, giving the MGE models of four galaxies used in JAMs (Section 6), is presented in this section as well. Also, the complete results of JAMs indicated for their systemic uncertainties are shown in Table 12.

Table 8

Gemini/NIFS Kinematic Data of the NGC 205 Nucleus

\begin{tabular}{lccc}
\hline \hline$\Delta$ R.A. $(\operatorname{arcsec})$ & $\Delta$ decl. $(\operatorname{arcsec})$ & $V_{\mathrm{r}}\left(\mathrm{km} \mathrm{s}^{-1}\right)$ & $\sigma\left(\mathrm{km} \mathrm{s}^{-1}\right)$ \\
\hline$\ldots$ & $\ldots$ & $\ldots$ & $\ldots$ \\
-0.10 & -0.09 & $-263.33 \pm 3.06$ & $15.26 \pm 2.20$ \\
-0.10 & 0.01 & $-269.34 \pm 1.10$ & $15.63 \pm 0.98$ \\
-0.15 & -0.04 & $-267.32 \pm 2.69$ & $16.34 \pm 2.65$ \\
$\ldots$ & $\ldots$ & $\ldots$ & $\ldots$ \\
\hline
\end{tabular}

(This table is available in its entirety in machine-readable form.)

Table 9

VLT/SINFONI Kinematic Data of the NGC 5102 Nucleus

\begin{tabular}{lccc}
\hline \hline$\Delta$ R.A. $(\operatorname{arcsec})$ & $\Delta$ decl. $(\operatorname{arcsec})$ & $V_{\mathrm{r}}\left(\mathrm{km} \mathrm{s}^{-1}\right)$ & $\sigma\left(\mathrm{km} \mathrm{s}^{-1}\right)$ \\
\hline$\ldots$ & $\ldots$ & $\ldots$ & $\ldots$ \\
0.02 & -0.02 & $492.93 \pm 1.29$ & $56.53 \pm 2.84$ \\
0.00 & 0.03 & $490.75 \pm 1.42$ & $56.04 \pm 3.26$ \\
-0.03 & -0.04 & $492.97 \pm 1.28$ & $52.16 \pm 2.36$ \\
$\ldots$ & $\ldots$ & $\ldots$ & $\ldots$ \\
\hline
\end{tabular}

(This table is available in its entirety in machine-readable form.)

Table 10

VLT/SINFONI Kinematic Data of the NGC 5206 Nucleus

\begin{tabular}{lccc}
\hline \hline$\Delta$ R.A. $(\operatorname{arcsec})$ & $\Delta$ decl. $(\operatorname{arcsec})$ & $V_{\mathrm{r}}\left(\mathrm{km} \mathrm{s}^{-1}\right)$ & $\sigma\left(\mathrm{km} \mathrm{s}^{-1}\right)$ \\
\hline$\ldots$ & $\ldots$ & $\ldots$ & $\ldots$ \\
0.01 & 0.02 & $610.18 \pm 2.55$ & $44.85 \pm 3.46$ \\
0.01 & -0.03 & $608.95 \pm 2.39$ & $44.57 \pm 2.76$ \\
0.01 & 0.07 & $607.90 \pm 2.07$ & $44.24 \pm 2.66$ \\
$\ldots$ & $\ldots$ & $\ldots$ & $\ldots$ \\
\hline
\end{tabular}

(This table is available in its entirety in machine-readable form.) 
Table 11

Luminosity and Mass MGE Models Used in Dynamical Modeling

\begin{tabular}{|c|c|c|c|c|}
\hline \multicolumn{5}{|c|}{ M32 } \\
\hline Mass & Light & $\sigma$ & $a / b$ & PA \\
\hline$\left(M_{\odot} / \mathrm{pc}^{2}\right)$ & $\left(L_{\odot} / \mathrm{pc}^{2}\right)$ & (arcsec) & & $\overline{(d e g)}$ \\
\hline (1) & (2) & (3) & (4) & (5) \\
\hline 5.01 & 4.74 & -2.76 & 0.75 & -25.0 \\
\hline 5.38 & 5.11 & -2.25 & 0.75 & -25.0 \\
\hline 5.64 & 5.37 & -1.78 & 0.75 & -25.0 \\
\hline 5.80 & 5.53 & -1.34 & 0.75 & -25.0 \\
\hline 5.85 & 5.58 & -0.94 & 0.75 & -25.0 \\
\hline 5.79 & 5.52 & -0.57 & 0.75 & -25.0 \\
\hline 5.62 & 5.35 & -0.23 & 0.75 & -25.0 \\
\hline 5.34 & 5.07 & 0.095 & 0.79 & -25.0 \\
\hline 4.93 & 4.66 & 0.398 & 0.79 & -25.0 \\
\hline 4.41 & 4.14 & 0.680 & 0.79 & -25.0 \\
\hline 3.77 & 3.50 & 0.946 & 0.79 & -25.0 \\
\hline 3.32 & 3.06 & 1.030 & 0.79 & -25.0 \\
\hline 3.00 & 2.73 & 1.195 & 0.79 & -25.0 \\
\hline 3.54 & 3.28 & 1.382 & 0.79 & -25.0 \\
\hline 3.19 & 2.92 & 1.611 & 0.79 & -25.0 \\
\hline 2.87 & 2.60 & 1.775 & 0.79 & -25.0 \\
\hline 2.71 & 2.44 & 1.907 & 0.79 & -25.0 \\
\hline 2.46 & 2.19 & 2.028 & 0.79 & -25.0 \\
\hline \multicolumn{5}{|c|}{ NGC 205} \\
\hline Mass & Light & $\sigma$ & $(a / b)$ & PA \\
\hline$\left(M_{\odot} / \mathrm{pc}^{2}\right)$ & $\left(L_{\odot} / \mathrm{pc}^{2}\right)$ & $(\operatorname{arcsec})$ & & (deg) \\
\hline (1) & (2) & (3) & (4) & (5) \\
\hline 4.87 & 4.58 & -1.75 & 0.95 & -40.4 \\
\hline 4.73 & 4.44 & -1.50 & 0.95 & -40.4 \\
\hline 5.23 & 4.94 & -1.15 & 0.95 & -40.4 \\
\hline 4.38 & 4.09 & -0.92 & 0.95 & -40.4 \\
\hline 4.93 & 4.64 & -0.68 & 0.95 & -40.4 \\
\hline 4.84 & 4.55 & -0.23 & 0.95 & -40.4 \\
\hline 4.36 & 4.07 & -0.00 & 0.95 & -40.4 \\
\hline 3.64 & 3.35 & 0.201 & 0.95 & -40.4 \\
\hline 3.62 & 3.33 & 0.871 & 0.90 & -40.4 \\
\hline 3.02 & 2.74 & 1.165 & 0.90 & -40.4 \\
\hline
\end{tabular}

Table 11

(Continued)

\begin{tabular}{|c|c|c|c|c|}
\hline \multicolumn{5}{|c|}{ NGC 5102} \\
\hline Mass & Light & $\sigma$ & $(a / b)$ & PA \\
\hline$\left(M_{\odot} / \mathrm{pc}^{2}\right)$ & $\left(L_{\odot} / \mathrm{pc}^{2}\right)$ & $(\operatorname{arcsec})$ & & $(\mathrm{deg})$ \\
\hline (1) & (2) & (3) & (4) & (5) \\
\hline 6.95 & 5.81 & -1.28 & 0.68 & 50.5 \\
\hline 6.70 & 5.26 & -1.11 & 0.68 & 50.5 \\
\hline 5.94 & 4.50 & -0.77 & 0.68 & 50.5 \\
\hline 4.95 & 3.51 & -0.40 & 0.68 & 50.5 \\
\hline 4.89 & 4.05 & -0.26 & 0.68 & 50.5 \\
\hline 4.77 & 3.93 & 0.25 & 0.60 & 50.5 \\
\hline 4.65 & 3.63 & 0.37 & 0.60 & 50.5 \\
\hline 3.47 & 2.46 & 0.82 & 0.60 & 50.5 \\
\hline 3.11 & 2.09 & 0.88 & 0.60 & 50.5 \\
\hline 2.88 & 2.04 & 1.38 & 0.60 & 50.5 \\
\hline 2.82 & 1.98 & 1.64 & 0.60 & 50.5 \\
\hline 2.48 & 1.63 & 1.75 & 0.60 & 50.5 \\
\hline 2.76 & 1.92 & 1.77 & 0.60 & 50.5 \\
\hline 2.47 & 1.62 & 1.32 & 0.60 & 50.5 \\
\hline \multicolumn{5}{|c|}{ NGC 5206} \\
\hline Mass & Light & $\sigma$ & $(a / b)$ & PA \\
\hline$\left(M_{\odot} / \mathrm{pc}^{2}\right)$ & $\left(L_{\odot} / \mathrm{pc}^{2}\right)$ & $(\operatorname{arcsec})$ & & (deg) \\
\hline (1) & (2) & (3) & (4) & (5) \\
\hline 5.60 & 5.49 & -1.74 & 0.96 & 38.3 \\
\hline 5.58 & 5.48 & -1.20 & 0.96 & 38.3 \\
\hline 5.43 & 5.33 & -1.11 & 0.96 & 38.3 \\
\hline 5.34 & 5.23 & -0.78 & 0.96 & 38.3 \\
\hline 4.16 & 4.06 & -0.66 & 0.96 & 38.3 \\
\hline 3.93 & 3.82 & -0.41 & 0.96 & 38.3 \\
\hline 3.83 & 3.72 & -0.27 & 0.96 & 38.3 \\
\hline 2.88 & 2.79 & -0.16 & 0.96 & 38.3 \\
\hline 2.98 & 2.88 & -0.08 & 0.98 & 38.3 \\
\hline 2.88 & 2.80 & 0.760 & 0.98 & 38.3 \\
\hline 2.61 & 2.73 & 0.890 & 0.98 & 38.3 \\
\hline 2.58 & 2.64 & 0.988 & 0.98 & 38.3 \\
\hline 2.40 & 2.33 & 1.316 & 0.98 & 38.3 \\
\hline 2.29 & 2.19 & 2.169 & 0.98 & 38.3 \\
\hline
\end{tabular}

Note. MGE models used in JAM fits in Section 6.1. Column 1: MGE models represented for galaxies' mass models. Column 2: MGE models of $K$-band luminosity densities, created from the Padova SSP linear fit color-color correlation models of the nuclei color HST/WFPC2 maps (Section 4.5). Column 3: Gaussian width along the major axis. Column 4: axial ratios $(a / b)$. Column 5: position angles. Columns 1,2, and 3 are shown in log scale. 
Table 12

JAM Results (Full Table)

\begin{tabular}{|c|c|c|c|c|c|c|c|c|c|c|}
\hline $\begin{array}{l}\text { Object } \\
\text { (1) }\end{array}$ & $\begin{array}{l}\text { Filter } \\
\text { (2) }\end{array}$ & $\begin{array}{c}\text { Color }-M / L \\
\text { (3) }\end{array}$ & $\begin{array}{c}M_{\mathrm{BH}} \\
\left(M_{\odot}\right) \\
(4)\end{array}$ & $\begin{array}{l}\beta_{\mathrm{z}} \\
(5)\end{array}$ & (6) & $\begin{array}{c}i \\
(\mathrm{deg}) \\
(7)\end{array}$ & $\begin{array}{c}\text { Number of Bins } \\
\text { (8) }\end{array}$ & $\begin{array}{l}\chi_{\mathrm{r}}^{2} \\
(9)\end{array}$ & $\begin{array}{c}r_{\mathrm{g}} \\
(\operatorname{arcsec}) \\
(10)\end{array}$ & $\begin{array}{c}r_{\mathrm{g}} \\
(\mathrm{pc}) \\
(11)\end{array}$ \\
\hline \multirow[t]{5}{*}{ M32 } & $\mathrm{F} 555 \mathrm{~W}^{\mathrm{G}}$ & R15 & $2.3_{-1.5}^{+1.3} \times 10^{6}$ & $-0.25_{-0.25}^{+0.35}$ & $1.05_{-0.15}^{+0.20}$ & $74_{-9}^{+12}$ & 1354 & 1.35 & 0.455 & 1.8 \\
\hline & $\mathrm{F} 814 \mathrm{~W}^{\mathrm{G}}$ & B03 & $2.0_{-0.8}^{+0.5} \times 10^{6}$ & $-0.15_{-0.35}^{+0.35}$ & $1.07_{-0.10}^{+0.14}$ & $72_{-6}^{+6}$ & & 1.31 & 0.337 & 1.35 \\
\hline & $\mathrm{F} 814 \mathrm{~W}^{\mathrm{G}}$ & Const & $2.0_{-1.0}^{+0.3} \times 10^{6}$ & $-0.20_{-0.40}^{+0.40}$ & $1.03_{-0.08}^{+0.17}$ & $70_{-5}^{+4}$ & & 1.97 & 0.337 & 1.35 \\
\hline & $\mathrm{F} 814 \mathrm{~W}^{\mathrm{D}}$ & $\mathrm{R} 15$ & $2.4 \times 10^{6}$ & -0.20 & 1.08 & 70 & & 1.75 & 0.387 & 1.61 \\
\hline & $\mathrm{F} 814 \mathrm{~W}^{\mathrm{D}}$ & Const & $2.1 \times 10^{6}$ & -0.20 & 1.03 & 70 & & 2.04 & 0.303 & 1.35 \\
\hline \multirow[t]{5}{*}{ NGC 205} & F555W $W^{\mathrm{D}}$ & $\mathrm{R} 15$ & $2.7 \times 10^{4}$ & 0.27 & 1.07 & 60 & 256 & 1.33 & 0.070 & 0.30 \\
\hline & $\mathrm{F}_{555 \mathrm{~W}^{\mathrm{G} *}}$ & R15 & $2.1 \times 10^{4}$ & 0.25 & 1.07 & 60 & & 1.37 & 0.051 & 0. \\
\hline & $\mathrm{F}_{555 \mathrm{~W}^{\mathrm{D}} *}$ & R15 & $3.0_{-3.0}^{+3.2} \times 10^{4}$ & $0.27_{-0.18}^{+0.08}$ & $1.07_{-0.20}^{+0.18}$ & $60_{-8}^{+5}$ & 197 & 1.37 & 0.074 & 0.35 \\
\hline & $\mathrm{F} 814 \mathrm{~W}^{\mathrm{D} *}$ & B03 & $2.0_{-2.0}^{+4.0} \times 10^{4}$ & $0.25_{-0.04}^{+0.06}$ & $1.09_{-0.10}^{+0.07}$ & $66_{-8}^{+4}$ & & 1.40 & 0.063 & 0.22 \\
\hline & $\mathrm{F} 814 \mathrm{~W}^{\mathrm{D} *}$ & Const & $1.8_{-1.8}^{+3.2} \times 10^{4}$ & $0.27_{-0.06}^{+0.04}$ & $1.07_{-0.07}^{+0.08}$ & $67_{-7}^{+4}$ & & 2.51 & 0.060 & 0.2 \\
\hline & F547M ${ }^{\mathrm{D}}$ & B03 & $7.5 \times 10^{5}$ & 0.18 & 1.13 & 76.0 & & 1.73 & 0.056 & 0.8 \\
\hline & F547M ${ }^{\mathrm{D}}$ & Const & $7.2 \times 10^{5}$ & 0.13 & 1.08 & 72.5 & & 2.92 & 0.048 & 0.82 \\
\hline \multirow[t]{7}{*}{ NGC 5206} & $\mathrm{~F} 555 \mathrm{~W}^{\mathrm{G}}$ & R15 & $5.1_{-1.2}^{+2.3} \times 10^{5}$ & $0.28_{-0.14}^{+0.07}$ & $1.08_{-0.40}^{+0.20}$ & $43.5_{-4.5}^{+4.0}$ & 240 & 1.33 & 0.061 & 1.04 \\
\hline & F555W $\mathrm{W}^{\mathrm{D}}$ & $\mathrm{R} 15$ & $4.8 \times 10^{5}$ & 0.28 & 1.08 & 43.5 & & 1.36 & 0.071 & 1.21 \\
\hline & $\mathrm{F} 814 \mathrm{~W}^{\mathrm{G}}$ & B03 & $2.4_{-1.3}^{+1.1} \times 10^{5}$ & $0.27_{-0.15}^{+0.04}$ & $1.10_{-0.10}^{+0.05}$ & $41.0_{-2.5}^{+2.5}$ & & 1.57 & 0.045 & 0.7 \\
\hline & $\mathrm{F} 814 \mathrm{~W}^{\mathrm{G}}$ & Const & $2.1_{-1.4}^{+0.8} \times 10^{5}$ & $0.29_{-0.04}^{+0.06}$ & $1.06_{-0.05}^{+0.10}$ & $45.0_{-1.5}^{+2.5}$ & & 2.41 & 0.038 & 0.65 \\
\hline & $\mathrm{F} 814 \mathrm{~W}^{\mathrm{D}}$ & R15 & $4.5 \times 10^{5}$ & 0.25 & 1.06 & 44.0 & & 1.70 & 0.058 & 1.00 \\
\hline & $\mathrm{F} 814 \mathrm{~W}^{\mathrm{D}}$ & B03 & $2.3 \times 10^{5}$ & 0.27 & 1.10 & 41.0 & & 1.66 & 0.045 & 0.75 \\
\hline & $\mathrm{F} 814 \mathrm{~W}^{\mathrm{D}}$ & Const & $2.0 \times 10^{5}$ & 0.29 & 1.06 & 45.0 & & 2.78 & 0.038 & 0.6 \\
\hline
\end{tabular}

Note. This table's notations are the same as in Table 6. Numbers without errors were fixed during JAM fitting. For NGC 205 , the filters with/without ${ }^{*}$ mean the bestfit models of after/before contaminated star subtraction of the NIFS kinematic spectroscopy.

\section{ORCID iDs}

Dieu D. Nguyen (1) https://orcid.org/0000-0002-5678-1008

Nadine Neumayer (i) https://orcid.org/0000-0002-6922-2598

Karina T. Voggel (i) https://orcid.org/0000-0001-6215-0950

Michele Cappellari iㅏ https://orcid.org/0000-0002-1283-8420

Victor Debattista (1) https://orcid.org/0000-0001-7902-0116

Nelson Caldwell (ib https://orcid.org/0000-0003-2352-3202

Christopher C. Ahn iㅣ https://orcid.org/0000-0002-9852-2258

\section{References}

Ahn, C. P., Seth, A. C., den Brok, M., et al. 2017, ApJ, 839, 72

Angus, G. W., Coppin, P., Gentile, G., \& Diaferio, A. 2016, MNRAS, 462,3221

Antonini, F. 2013, ApJ, 763, 62

Antonini, F., Barausse, E., \& Silk, J. 2015a, ApJ, 812, 72

Antonini, F., Barausse, E., \& Silk, J. 2015b, ApJL, 806, L8

Avila, R. J., Hack, W. J. \& STScI AstroDrizzle Team 2012, AAS Meeting, $220,135.13$

Bahcall, J. N., \& Wolf, R. A. 1977, ApJ, 216, 883

Bailey, M. E. 1980, MNRAS, 191, 195

Baldassare, V. F., Reines, A. E., Gallo, E., \& Greene, J. E. 2015, ApJL, 809, L14

Barth, A. J., Ho, L. C., Rutledge, R. E., \& Sargent, W. L. W. 2004, ApJ, 607, 90

Bell, E. F., \& de Jong, R. S. 2001, ApJ, 550, 212

Bell, E. F., McIntosh, D. H., Katz, N., \& Weinberg, M. D. 2003, ApJS, 149,289
Bianchini, P., Sills, A., van de Ven, G., \& Sippel, A. C. 2017, MNRAS, 469,4359

Blanton, M. R., Lupton, R. H., Schlegel, D. J., et al. 2005, ApJ, 631, 208

Bonnet, H., Abuter, R., Baker, A., et al. 2004, Msngr, 117, 17

Bonoli, S., Mayer, L., \& Callegari, S. 2014, MNRAS, 437, 1576

Bressan, A., Marigo, P., Girardi, L., et al. 2012, MNRAS, 427, 127

Bruzual, G., \& Charlot, S. 2003, MNRAS, 344, 1000

Butler, D. J., \& Martínez-Delgado, D. 2005, AJ, 129, 2217

Caldwell, N., \& Bothun, G. D. 1987, AJ, 94, 1126

Cappellari, M. 2008, MNRAS, 390, 71

Cappellari, M. 2017, MNRAS, 466, 798

Cappellari, M., Bacon, R., Bureau, M., et al. 2006, MNRAS, 366, 1126

Cappellari, M., Bertola, F., Burstein, D., et al. 1999, ApJL, 515, L17

Cappellari, M., \& Copin, Y. 2003, MNRAS, 342, 345

Cappellari, M., \& Emsellem, E. 2004, PASP, 116, 138

Cappellari, M., Neumayer, N., Reunanen, J., et al. 2009, MNRAS, 394, 660

Cardelli, J. A., Clayton, G. C., \& Mathis, J. S. 1989, ApJ, 345, 245

Carson, D. J., Barth, A. J., Seth, A. C., et al. 2015, AJ, 149, 170

Coelho, P., Mendes de Oliveira, C., \& Cid Fernandes, R. 2009, MNRAS, 396, 624

Corbin, M. R., O’Neil, E., \& Rieke, M. J. 2001, AJ, 121, 2549

Côté, P., Piatek, S., Ferrarese, L., et al. 2006, ApJS, 165, 57

Davidge, T. J. 2003, ApJ, 597, 289

Davidge, T. J. 2005, AJ, 130, 2087

Davidge, T. J. 2008, AJ, 135, 1636

Davidge, T. J. 2015, ApJ, 799, 97

De Rijcke, S., Prugniel, P., Simien, F., \& Dejonghe, H. 2006, MNRAS, 369,1321

Deharveng, J.-M., Jedrzejewski, R., Crane, P., Disney, M. J., \& RoccaVolmerange, B. 1997, A\&A, 326, 528

den Brok, M., Peletier, R. F., Seth, A., et al. 2014a, MNRAS, 445, 2385

den Brok, M., Seth, A. C., Barth, A. J., et al. 2015, ApJ, 809, 101 
den Brok, M., van de Ven, G., van den Bosch, R., \& Watkins, L. 2014b, MNRAS, 438, 487

Dong, X.-B., Ho, L. C., Yuan, W., et al. 2012, ApJ, 755, 167

Drehmer, D. A., Storchi-Bergmann, T., Ferrari, F., Cappellari, M., \& Riffel, R. A. 2015, MNRAS, 450, 128

Dressler, A., \& Richstone, D. O. 1988, ApJ, 324, 701

Eisenhauer, F., Abuter, R., Bickert, K., et al. 2003, Proc. SPIE, 4841, 1548

Emsellem, E., Monnet, G., \& Bacon, R. 1994a, A\&A, 285, 723

Emsellem, E., Monnet, G., Bacon, R., \& Nieto, J.-L. 1994b, A\&A, 285, 739

Fabian, A. C. 2012, ARA\&A, 50, 455

Feldmeier-Krause, A., Kerzendorf, W., Neumayer, N., et al. 2017, MNRAS, 464, 194

Ferrarese, L., Côté, P., Dalla Bontà, E., et al. 2006, ApJL, 644, L21

Gallo, E., Treu, T., Jacob, J., et al. 2008, ApJ, 680, 154

Gebhardt, K., Lauer, T. R., Kormendy, J., et al. 2001, AJ, 122, 2469

Geha, M., Guhathakurta, P., Rich, R. M., \& Cooper, M. C. 2006, AJ, 131, 332

Georgiev, I. Y., \& Böker, T. 2014, MNRAS, 441, 3570

Georgiev, I. Y., Böker, T., Leigh, N., Lützgendorf, N., \& Neumayer, N. 2016, MNRAS, 457, 2122

Graham, A. W. 2002, ApJL, 568, L13

Graham, A. W., \& Scott, N. 2015, ApJ, 798, 54

Graham, A. W., \& Spitler, L. R. 2009, MNRAS, 397, 2148

Greene, J. E. 2012, NatCo, 3, 1304

Greene, J. E., \& Ho, L. C. 2007, ApJ, 670, 92

Greene, J. E., Seth, A., Kim, M., et al. 2016, ApJL, 826, L32

Guillard, N., Emsellem, E., \& Renaud, F. 2016, MNRAS, 461, 3620

Haşegan, M., Jordán, A., Côté, P., et al. 2005, ApJ, 627, 203

Häring, N., \& Rix, H.-W. 2004, ApJL, 604, L89

Ho, L. C., Greene, J. E., Filippenko, A. V., \& Sargent, W. L. W. 2009, ApJS, 183,1

Ho, L. C., Li, Z.-Y., Barth, A. J., Seigar, M. S., \& Peng, C. Y. 2011, ApJS, 197, 21

Ho, L. C., Terashima, Y., \& Ulvestad, J. S. 2003, ApJ, 589, 783

Hopkins, P. F., Lauer, T. R., Cox, T. J., Hernquist, L., \& Kormendy, J. 2009, ApJS, 181, 486

Hopkins, P. F., \& Quataert, E. 2010, MNRAS, 405, L41

Huang, S., Ho, L. C., Peng, C. Y., Li, Z.-Y., \& Barth, A. J. 2013, ApJ, 766, 47

Husser, T.-O., Wende-von Berg, S., Dreizler, S., et al. 2013, A\&A, 553, A6

Jarrett, T. H., Chester, T., Cutri, R., Schneider, S. E., \& Huchra, J. P. 2003, AJ, 125,525

Jedrzejewski, R. I. 1987, MNRAS, 226, 747

Kamann, S., Wisotzki, L., \& Roth, M. M. 2013, A\&A, 549, A71

Karachentsev, I. D., Makarov, D. I., \& Kaisina, E. I. 2013, AJ, 145, 101

Kent, S. M. 1987, AJ, 94, 306

Kochanek, C. S. 2016, MNRAS, 461, 371

Kormendy, J., \& Bender, R. 2009, ApJL, 691, L142

Kormendy, J., \& Ho, L. C. 2013, ARA\&A, 51, 511

Kraft, R. P., Nolan, L. A., Ponman, T. J., Jones, C., \& Raychaudhury, S. 2005, ApJ, 625, 785

Krajnović, D., Cappellari, M., de Zeeuw, P. T., \& Copin, Y. 2006, MNRAS, 366, 787

Krajnović, D., Cappellari, M., \& McDermid, R. M. 2018, MNRAS, 473, 5237

Krist, J. 1995, in ASP Conf. Ser. 77, Astronomical Data Analysis Software and Systems IV, ed. R. A. Shaw, H. E. Payne, \& J. J. E. Hayes (San Francisco, CA: ASP), 349

Krist, J. E., Hook, R. N., \& Stoehr, F. 2011, Proc. SPIE, 8127, 81270J

Läsker, R., Greene, J. E., Seth, A., et al. 2016a, ApJ, 825, 3

Läsker, R., Greene, J. E., Seth, A., et al. 2016b, ApJ, 825, 3

Lauer, T. R., Faber, S. M., Ajhar, E. A., Grillmair, C. J., \& Scowen, P. A. 1998, AJ, 116, 2263

Law-Smith, J., Ramirez-Ruiz, E., Ellison, S. L., \& Foley, R. J. 2017, ApJ, 850,22

Leigh, N. W. C., Antonini, F., Stone, N. C., Shara, M. M., \& Merritt, D. 2016, MNRAS, 463, 1605

Leung, G. Y. C., Leaman, R., van de Ven, G., et al. 2018, MNRAS, 477,254

Li, H., Ge, J., Mao, S., et al. 2017, ApJ, 838, 77

Li, Z.-Y., Ho, L. C., Barth, A. J., \& Peng, C. Y. 2011, ApJS, 197, 22

Lodato, G., \& Natarajan, P. 2006, MNRAS, 371, 1813

Lotz, J. M., Telford, R., Ferguson, H. C., et al. 2001, ApJ, 552, 572

Lyubenova, M., van den Bosch, R. C. E., Côté, P., et al. 2013, MNRAS, 431, 3364

Maksym, W. P., Ulmer, M. P., Eracleous, M. C., Guennou, L., \& Ho, L. C. 2013, MNRAS, 435, 1904

Marconi, A., Risaliti, G., Gilli, R., et al. 2004, MNRAS, 351, 169
Markwardt, C. B. 2009, in ASP Conf. Ser. 411, Astronomical Data Analysis Software and Systems XVIII, ed. D. A. Bohlender, D. Durand, \& P. Dowler (San Francisco, CA: ASP), 251

McConnachie, A. W. 2012, AJ, 144, 4

McConnachie, A. W., Irwin, M. J., Ferguson, A. M. N., et al. 2005, MNRAS, 356, 979

McConnell, N. J., \& Ma, C.-P. 2013, ApJ, 764, 184

McMillan, R., Ciardullo, R., \& Jacoby, G. H. 1994, AJ, 108, 1610

Merritt, D. 2013, Dynamics and Evolution of Galactic Nuclei (Princeton, NJ: Princeton Univ. Press)

Mieske, S., Frank, M. J., Baumgardt, H., et al. 2013, A\&A, 558, A14

Miller, B. P., Gallo, E., Greene, J. E., et al. 2015, ApJ, 799, 98

Mitzkus, M., Cappellari, M., \& Walcher, C. J. 2017, MNRAS, 464, 4789

Monaco, L., Bellazzini, M., Ferraro, F. R., \& Pancino, E. 2005, MNRAS, 356, 1396

Monaco, L., Saviane, I., Perina, S., et al. 2009, A\&A, 502, L9

Moran, E. C., Shahinyan, K., Sugarman, H. R., Vélez, D. O., \& Eracleous, M. 2014, AJ, 148, 136

Natarajan, P., Pacucci, F., Ferrara, A., et al. 2017, ApJ, 838, 117

Nayakshin, S., Wilkinson, M. I., \& King, A. 2009, MNRAS, 398, L54

Neumayer, N., Cappellari, M., Reunanen, J., et al. 2007, ApJ, 671, 1329

Neumayer, N., \& Walcher, C. J. 2012, AdAst, 2012, 709038

Nguyen, D. D., Seth, A. C., den Brok, M., et al. 2017, ApJ, 836, 237

Nguyen, D. D., Seth, A. C., Reines, A. E., et al. 2014, ApJ, 794, 34

Norris, M. A., Kannappan, S. J., Forbes, D. A., et al. 2014, MNRAS, 443,1151

Paudel, S., Lisker, T., \& Kuntschner, H. 2011, MNRAS, 413, 1764

Peng, C. Y., Ho, L. C., Impey, C. D., \& Rix, H.-W. 2010, AJ, 139, 2097

Peterson, R. C., \& Caldwell, N. 1993, AJ, 105, 1411

Pfeffer, J., Griffen, B. F., Baumgardt, H., \& Hilker, M. 2014, MNRAS, 444, 3670

Poci, A., Cappellari, M., \& McDermid, R. M. 2017, MNRAS, 467, 1397

Portegies Zwart, S. F., Baumgardt, H., Hut, P., Makino, J., \& McMillan, S. L. W. 2004, Natur, 428, 724

Pritchet, C. 1979, ApJ, 231, 354

Reines, A. E., \& Comastri, A. 2016, PASA, 33, e054

Reines, A. E., Greene, J. E., \& Geha, M. 2013, ApJ, 775, 116

Reines, A. E., \& Volonteri, M. 2015, ApJ, 813, 82

Richstone, D., \& Sargent, W. L. W. 1972, ApJ, 176, 91

Roediger, J. C., \& Courteau, S. 2015, MNRAS, 452, 3209

Rose, J. A., Arimoto, N., Caldwell, N., et al. 2005, AJ, 129, 712

Rossa, J., van der Marel, R. P., Böker, T., et al. 2006, AJ, 132, 1074

Saglia, R. P., Opitsch, M., Erwin, P., et al. 2016, ApJ, 818, 47

Satyapal, S., Böker, T., Mcalpine, W., et al. 2009, ApJ, 704, 439

Schinnerer, E., Böker, T., \& Meier, D. S. 2003, ApJL, 591, L115

Schlafly, E. F., \& Finkbeiner, D. P. 2011, ApJ, 737, 103

Schödel, R., Feldmeier, A., Kunneriath, D., et al. 2014, A\&A, 566, A47

Schödel, R., Merritt, D., \& Eckart, A. 2009, A\&A, 502, 91

Scott, N., Graham, A. W., \& Schombert, J. 2013, ApJ, 768, 76

Seth, A., Agüeros, M., Lee, D., \& Basu-Zych, A. 2008, ApJ, 678, 116

Seth, A. C. 2010 , ApJ, 725, 670

Seth, A. C., Cappellari, M., Neumayer, N., et al. 2010, ApJ, 714, 713

Seth, A. C., Dalcanton, J. J., Hodge, P. W., \& Debattista, V. P. 2006, AJ, 132,2539

Seth, A. C., van den Bosch, R., Mieske, S., et al. 2014, Natur, 513, 398

Shanahan, R. L., \& Gieles, M. 2015, MNRAS, 448, L94

Sharina, M. E., Afanasiev, V. L., \& Puzia, T. H. 2006, MNRAS, 372, 1259

She, R., Ho, L. C., \& Feng, H. 2017, ApJ, 842, 131

Sirianni, M., Jee, M. J., Benítez, N., et al. 2005, PASP, 117, 1049

Spengler, C., Côté, P., Roediger, J., et al. 2017, ApJ, 849, 55

Stone, N. C., Küpper, A. H. W., \& Ostriker, J. P. 2017, MNRAS, 467, 4180

Stone, N. C., \& Metzger, B. D. 2016, MNRAS, 455, 859

Thater, S., Krajnović, D., Bourne, M. A., et al. 2017, A\&A, 597, A18

Thornton, C. E., Barth, A. J., Ho, L. C., Rutledge, R. E., \& Greene, J. E. 2008, ApJ, 686, 892

Tully, R. B., Libeskind, N. I., Karachentsev, I. D., et al. 2015, ApJL, 802, L25 Valluri, M., Ferrarese, L., Merritt, D., \& Joseph, C. L. 2005, ApJ, 628, 137 van den Bergh, S. 1976, AJ, 81, 795

van den Bosch, R. C. E., \& de Zeeuw, P. T. 2010, MNRAS, 401, 1770

van den Bosch, R. C. E., van de Ven, G., Verolme, E. K., Cappellari, M., \& de Zeeuw, P. T. 2008, MNRAS, 385, 647

van Wassenhove, S., Volonteri, M., Walker, M. G., \& Gair, J. R. 2010 , MNRAS, 408, 1139

van Woerden, H., Schwarz, U. J., Peletier, R. F., \& Wakker, B. P. 1996, in IAU Symp. 171, New Light on Galaxy Evolution, ed. R. Bender \& R. L. Davies (Dordrecht: Kluwer), 464 
Verolme, E. K., Cappellari, M., Copin, Y., et al. 2002, MNRAS, 335, 517

Villaume, A., Brodie, J., Conroy, C., Romanowsky, A. J., \& van Dokkum, P. 2017, ApJL, 850, L14

Volonteri, M. 2010, A\&ARv, 18, 279

Volonteri, M., Lodato, G., \& Natarajan, P. 2008, MNRAS, 383, 1079

Volonteri, M., Reines, A. E., Atek, H., Stark, D. P., \& Trebitsch, M. 2017, ApJ, 849,155

Walcher, C. J., Böker, T., Charlot, S., et al. 2006, ApJ, 649, 692

Walcher, C. J., van der Marel, R. P., McLaughlin, D., et al. 2005, ApJ, 618,237
Wallace, L., \& Hinkle, K. 1996, ApJS, 107, 312

Wegner, G., Bernardi, M., Willmer, C. N. A., et al. 2003, AJ, 126, 2268

Weigel, A. K., Schawinski, K., Treister, E., et al. 2015, MNRAS, 448, 3167

Welch, D. L., Madore, B. F., McAlary, C. W., \& McLaren, R. A. 1986, ApJ, 305,583

Wevers, T., van Velzen, S., Jonker, P. G., et al. 2017, MNRAS, 471, 1694

Worthey, G. 2004, AJ, 128, 2826

Xilouris, E. M., Madden, S. C., Galliano, F., Vigroux, L., \& Sauvage, M. 2004, A\&A, 416, 41

Yang, Y., Li, Z., Sjouwerman, L. O., et al. 2015, ApJL, 807, L19 